Supporting Information

\title{
Characterization of Peroxo and Hydroperoxo Intermediates in the Aerobic Oxidation of $\mathrm{N}$-Heterocyclic-Carbene-Coordinated Palladium(0)
}

\author{
Michael M. Konnick, Ilia A. Guzei, and Shannon S. Stahl* \\ Department of Chemistry, University of Wisconsin-Madison, 1101 University \\ Avenue, Madison, WI 53706
}

\section{Table of Contents}

Experimental: General Considerations

Preparation and characterization of $\mathrm{Pd}(\mathrm{IMes})_{2}$ (2)

Preparation and characterization of $\mathrm{Pd}(\mathrm{IMes})_{2}\left(\mathrm{O}_{2}\right)(\mathbf{3})$, and $\mathrm{Pd}(\mathrm{IMes})_{2}\left({ }^{18} \mathrm{O}_{2}\right)\left(\mathbf{3 -}{ }^{\mathbf{1 8}} \mathbf{O}_{2}\right)$

Titration of HOAc against $\mathrm{Pd}(\mathrm{IMes})_{2}\left(\mathrm{O}_{2}\right)$

Preparation and characterization of $\mathrm{Pd}(\mathrm{IMes})_{2}(\mathrm{OOH})(\mathrm{OAc})(4)$,

$\mathrm{Pd}(\mathrm{IMes})_{2}\left({ }^{18} \mathrm{O}^{18} \mathrm{OH}\right)(\mathrm{OAc})\left(\mathbf{4}-{ }^{\mathbf{1 8}} \mathrm{O}\right)$, and $\mathrm{Pd}(\mathrm{IMes})_{2}(\mathrm{OOD})(\mathrm{OAc})\left(\mathbf{4 - \boldsymbol { d } _ { \mathbf { 1 } } )}\right.$

Independent synthesis of $\mathrm{Pd}(\mathrm{IMes})_{2}(\mathrm{OAc})_{2}$ (5)

Comparison of carbene ${ }^{13} \mathrm{C}$-resonances of (2), (3), (4), and (5)

Spectrophotometric detection of $\mathrm{H}_{2} \mathrm{O}_{2}$ in the protonolysis of $\mathrm{Pd}(\mathrm{IMes})_{2}\left(\mathrm{O}_{2}\right)$

X-ray crystallographic analysis of $\mathrm{Pd}(\mathrm{IMes})_{2}$ (2)

X-ray crystallographic analysis of $\mathrm{Pd}(\mathrm{IMes})_{2}\left(\mathrm{O}_{2}\right)(\mathbf{3})$

$\mathrm{X}$-ray crystallographic analysis of $\mathrm{Pd}(\mathrm{IMes})_{2}(\mathrm{OAc})_{2}$ (5)
Pages

S2

S3

S4-S5

S6-S7

S8-S12

S13

S14

S14-S15

S16-S26

S27-S42

S43-S52 


\section{Experimental}

General Considerations. All procedures, except the oxygenation reactions, were carried out under an inert atmosphere of nitrogen in a MBraun glove box or by using standard Schlenk techniques. NMR spectral data was obtained using Bruker AC-300 or AM-360 MHz NMR spectrometers. ${ }^{1} \mathrm{H}-\mathrm{NMR}$ spectra were referenced to residual $\mathrm{C}_{6} \mathrm{D}_{5} \mathrm{H}(7.16 \mathrm{ppm})$, and ${ }^{13} \mathrm{C}-\mathrm{NMR}$ spectra were referenced to the $\mathrm{C}_{6} \mathrm{D}_{6}$ resonance $(128.39 \mathrm{ppm})$. Infrared spectra were obtained with a Bruker Tensor 27 spectrometer equipped with a single reflection MIRacle Horizontal ATR ZnSe crystal by Pike Technologies. The matrix-assisted laser desorption/ionization (MALDI) mass spectra were obtained using a Bruker REFLEX II equipped with a $337 \mathrm{~nm}$ laser, a reflectron, and delayed extraction in the positive ion mode with an acceleration voltage of $25 \mathrm{kV}$. Electrospray ionization mass spectra were obtained with a Micromass LCT, utilizing a time-of-flight analyzer. All ESI-TOF samples were dissolved in methanol and sprayed with a sample cone voltage of $20 \mathrm{~V}$. All solvents used were dried and deoxygenated prior to use: $\mathrm{C}_{6} \mathrm{H}_{6}$, $\mathrm{C}_{6} \mathrm{D}_{6}$, toluene and pentane were degassed via standard freeze/pump/thaw methods and then dried over $\mathrm{Na}$ /benzophenone, $\mathrm{CH}_{2} \mathrm{Cl}_{2}$ and diethyl ether were passed thorough a column of activated alumina. The complex $\operatorname{Pd}(\mathrm{IMes})_{2}$ was synthesized following published literature procedures. ${ }^{1}$ Acetic acid, acetic acid- $d_{1}$, methanol- $d_{4}$, and 1,3,5-( $\left.{ }^{t} \mathrm{Bu}\right)_{3}-\mathrm{C}_{6} \mathrm{H}_{3}$ was purchased from Aldrich and ${ }^{18} \mathrm{O}$-labeled dioxygen was purchased from Isotech, Inc. These items were used as received.

${ }^{1}$ Böhm, V. P. W.; Gstöttmayr, C. W. K.; Weskamp, T.; Herrmann, W. A., J. Organomet. Chem. 2000, 595, 186-190. 
Preparation of Pd(IMes) 2 (2). Published literature procedures were followed ${ }^{1}$ with the corrected NMR characterization data provided below. Recrystallization of the lemon yellow $\mathrm{Pd}(\mathrm{IMes})_{2}$ for X-ray diffraction analysis was accomplished by slow evaporation of a diethyl ether solution.

${ }^{1} \mathrm{H}$ NMR $\left(300 \mathrm{MHz}, \mathrm{C}_{6} \mathrm{D}_{6}\right): \delta 6.82(\mathrm{~s}, 8 \mathrm{H}), 6.14(\mathrm{~s}, 4 \mathrm{H}), 2.34(\mathrm{~s}, 12 \mathrm{H}), 2.04(\mathrm{~s}, 24 \mathrm{H}) ;{ }^{13} \mathrm{C} \mathrm{NMR}$ (75 MHz, $\left.\mathrm{C}_{6} \mathrm{D}_{6}\right)$ : $\delta 19.1$ (o-Me), 21.7 ( $p$-Me), 119.1 (-HC=CH-), 129.0, 136.1, 136.5, 139.2 (aryl C), 198.9 (N-C-N); MS (MALDI-TOF): $714.3[\mathrm{M}]^{+}$; X-ray: (see data and tables below)

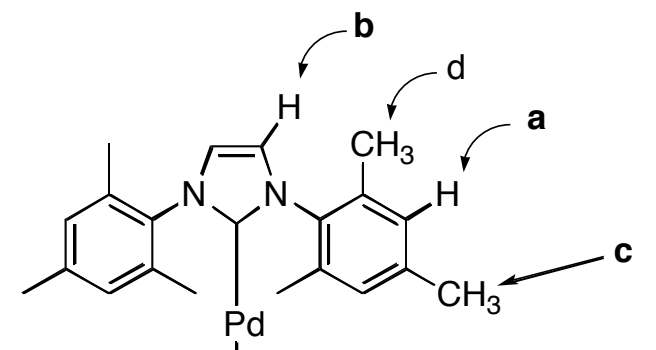<smiles>Cc1cc(C)c(N2C=CN(c3c(C)cc(C)cc3C)C2O)c(C)c1</smiles>

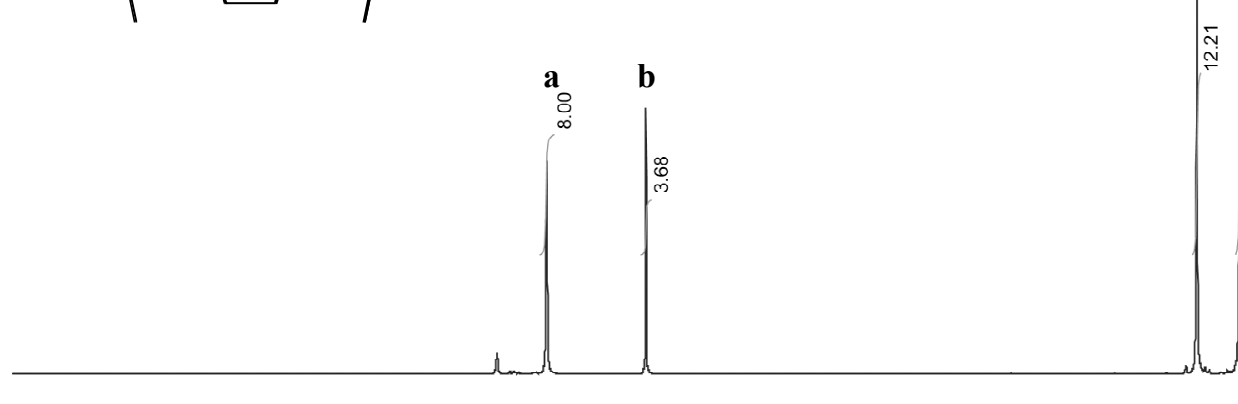

Figure S1. ${ }^{1} \mathrm{H}-\mathrm{NMR}$ spectrum of (2). 
Preparation of $\mathbf{P d}(\mathrm{IMes})_{2}\left(\mathbf{O}_{2}\right)$ (3). As a representative experiment, $\mathrm{Pd}(\mathrm{IMes})_{2}(120 \mathrm{mg}$, $168 \mathrm{mmol}$ ) was dissolved in $15 \mathrm{~mL}$ of toluene in a Schlenk tube equipped with a teflon valve and removed from the glove box. The flask was evacuated and refilled with an atmosphere of dry $\mathrm{O}_{2}$ at which time the solution immediately changed color from lemon yellow to brown. After allowing the solution to stir under $\mathrm{O}_{2}$ for 15 minutes, the solution was filtered through celite, and the solvent was removed under vacuum to yield a pure dark brown solid (97\%). X-ray quality crystals were obtained by slow diffusion of pentane into a saturated toluene solution.

Analytical Data:

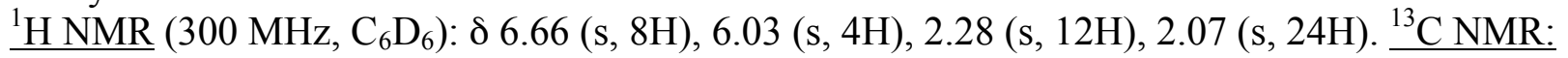
(75 MHz, $\left.\mathrm{C}_{6} \mathrm{D}_{6}\right): \delta 19.0(o-\mathrm{Me}), 21.8(p-\mathrm{Me}), 123.8(-\mathrm{HC}=\mathbf{C H}-), 129.5,136.4,137.5,137.8$ (aryl C), 187.7 (N-C-N); IR (ATR) $v_{\max }\left(\mathrm{cm}^{-1}\right): 1489,1401,1308,1273,1265,1084,1077,1033,926$, 867 (m, O-O), 852, 759, 757, 740, 734, 727, 699, 694; MS (MALDI-TOF) $\mathrm{m} / z: 714.3$ [M - $\left.\mathrm{O}_{2}\right]^{+}$; MS (ESI-TOF) $\mathrm{m} / z$ (relative intensity): 747.1(100) $[\mathrm{M}+\mathrm{H}]^{+}, 714.1(31)\left[\mathrm{M}^{-} \mathrm{O}_{2}\right]^{+}, 305.1(4)$

$[\mathrm{IMesH}]^{+} ; \underline{\mathrm{X} \text {-ray: }}$ (see data and tables below)

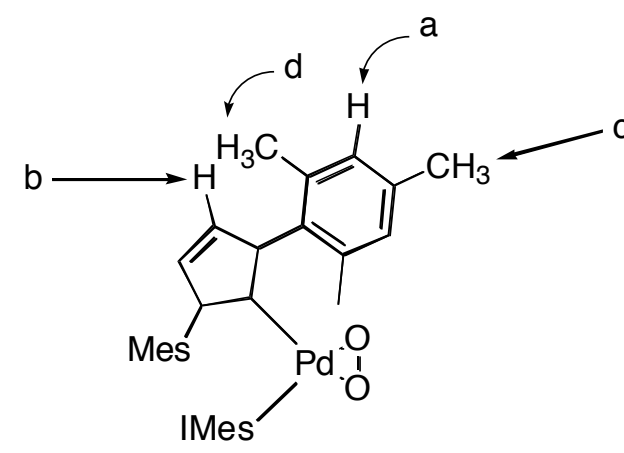

IMes =<smiles>Cc1cc(C)c(C)c(C)c1</smiles>

d

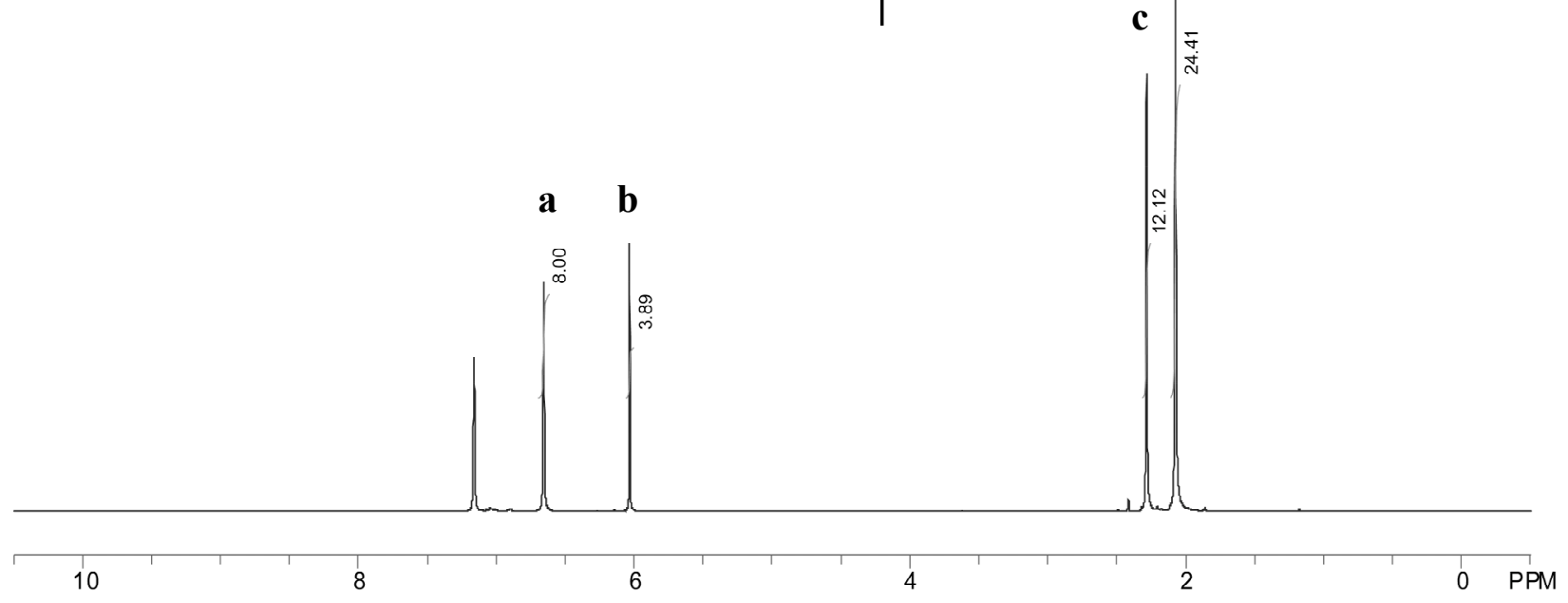

Figure S2. ${ }^{1} \mathrm{H}-\mathrm{NMR}$ spectrum of (3). 
Preparation of $\operatorname{Pd}(\mathrm{IMes})_{2}\left({ }^{18} \mathrm{O}_{2}\right),\left(3^{-18} \mathrm{O}\right)$. A procedure similar to that for 3 was followed except ${ }^{18} \mathrm{O}_{2}$ was introduced via a lecture bottle. The flask was cooled with liquid nitrogen and the contents were placed under vacuum. The lecture bottle was opened to introduce an ${ }^{18} \mathrm{O}_{2}$ atmosphere over the solution. The solution was warmed to room temperature and stirred for 1 hour. Isolation of $\mathbf{3}^{18} \mathbf{O}$ was performed in an analogous manner as that for $\mathbf{3}$.

Analytical Data:

IR (ATR) $v_{\max }\left(\mathrm{cm}^{-1}\right)$ : identical to 3 except minus 867 and plus 821 . Infrarred spectroscopic data for $\mathbf{3}$ and $\mathbf{3}^{-18} \mathrm{O}$ are shown in Figure S3.

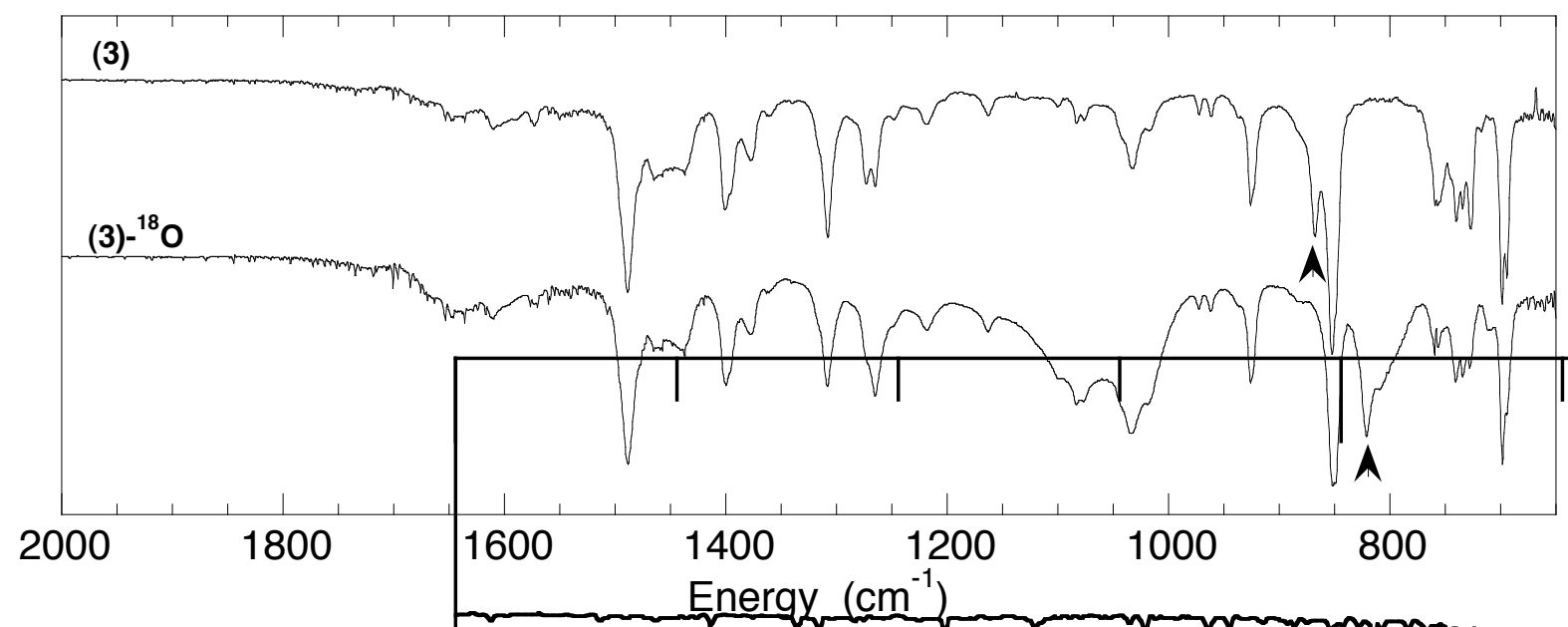

Figure S3. FTIR spectra of $\mathbf{3}$ and $\mathbf{3}^{18} \mathbf{O}$. The O-O stretching vibrations are maked with arrows.

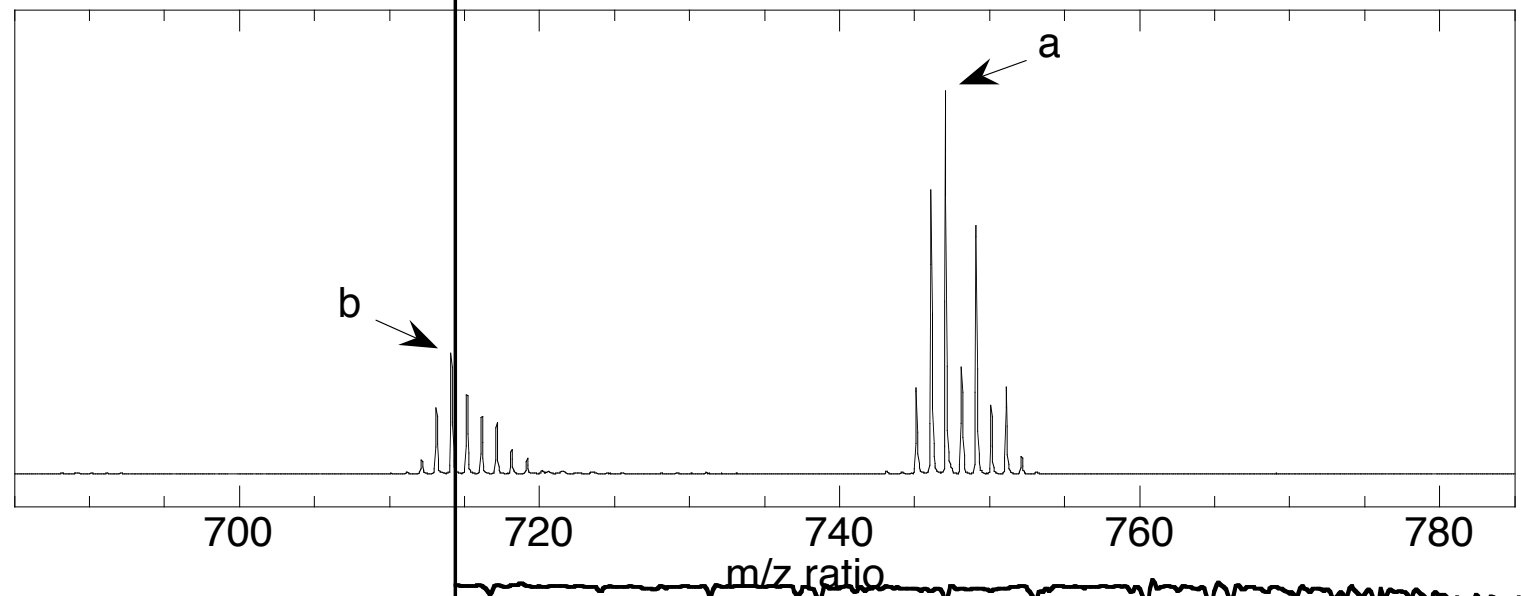

Figure S4. ESI-TOF mass spectum of $\mathbf{3}$, focusing on the $\mathrm{m} / \mathrm{z}=700-780$ region. The major peaks (a) correspond to the $[\mathrm{M}+\mathrm{H}]^{+}$ion $(\mathrm{m} / \mathrm{z}=747.1)$ and the lower intensity peaks $(\mathbf{b})$ correspond to the $\left[\mathrm{M}-\mathrm{O}_{2}\right]^{+}$ion $(\mathrm{m} / \mathrm{z}=714.1)$. Non-ideal isotopic mass distribution in $(\mathbf{b})$ is attributed to the presence of the $\left[\mathrm{Pd}(\mathrm{IMes})_{2}(\mathrm{H})\right]^{+}$ion $(\mathrm{m} / \mathrm{z}=715.1)$ which is present as the predominant mass fragment when spectra are obtained at higher concentrations of (3). 
Protonolysis of Pd(IMes) $)_{2}\left(\mathrm{O}_{2}\right)(3)$ with HOAc. An NMR tube equipped with a septum was charged with $\mathrm{Pd}(\mathrm{IMes})_{2}\left(\mathrm{O}_{2}\right)(1.7 \mathrm{mg}, 2.3 \mu \mathrm{mol}), 1,3,5-\left({ }^{t} \mathrm{Bu}\right)_{3}-\mathrm{C}_{6} \mathrm{H}_{3} / \mathrm{C}_{6} \mathrm{D}_{6}(500 \mu \mathrm{L}$ solution, $13.3 \mathrm{mM}, 6.63 \mu \mathrm{mol})$ as an internal standard, and removed from the glove box. A volumetric flask equipped with a septum was charged with a $1 \mathrm{~mL}$ solution of $\mathrm{HOAc}$ in $\mathrm{C}_{6} \mathrm{D}_{6}(10.5 \mu \mathrm{L}$ HOAc, $0.183 \mathrm{M}$ ) and removed from the glove box. A ${ }^{1} \mathrm{H}$ NMR spectrum was obtained of $\mathrm{Pd}(\mathrm{IMes})_{2}\left(\mathrm{O}_{2}\right)$ before addition of HOAc, and additional spectra were obtained after each HOAc addition. After a total addition of 2.5 equivalents of HOAc, spectra were obtained at regular intervals over a period of 3 days (see spectra on next page). 

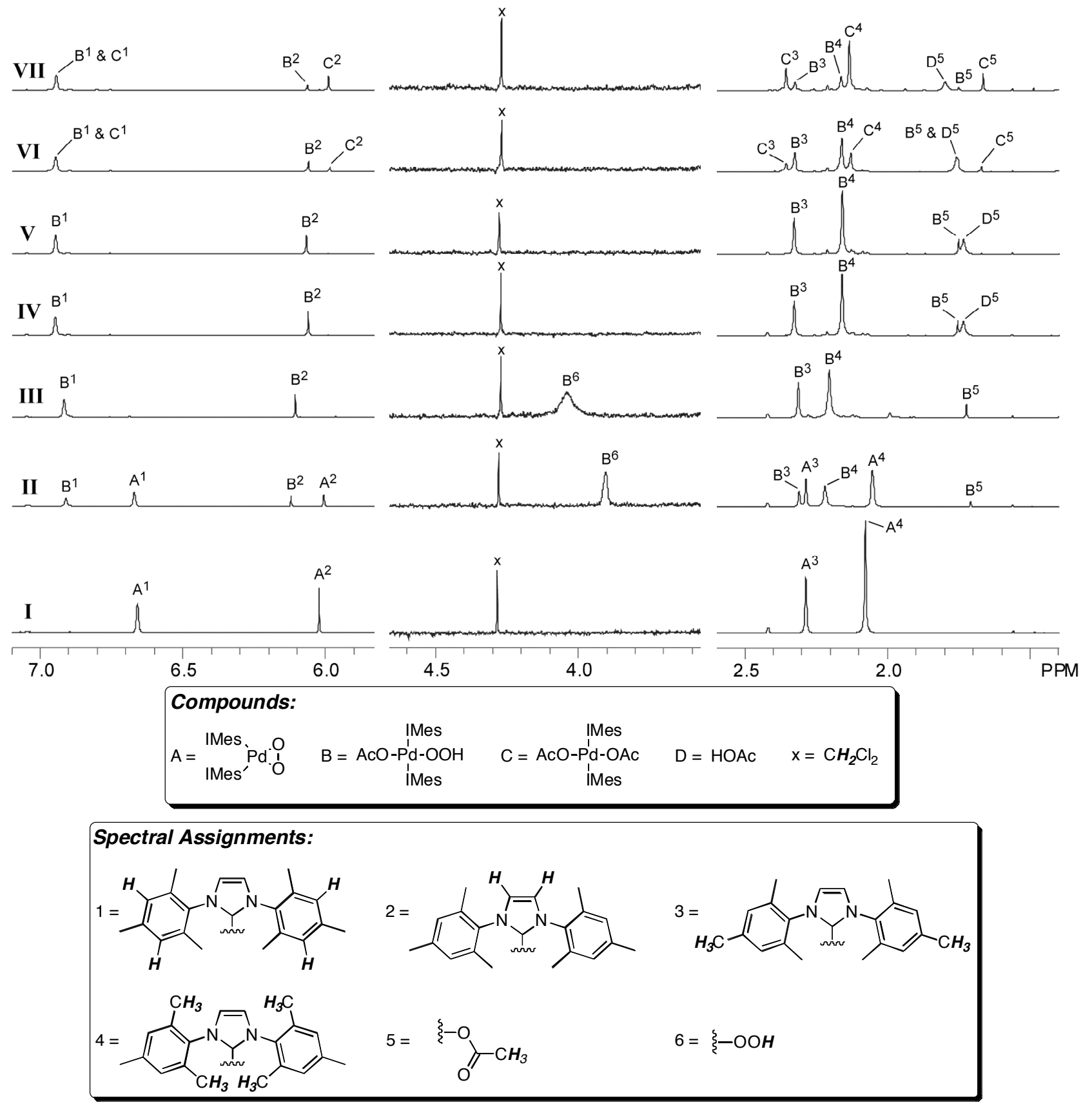

Figure S5. Titration of HOAc against $\mathrm{Pd}(\mathrm{IMes})_{2}(\mathrm{O})_{2}(3)$ in $\mathrm{C}_{6} \mathrm{D}_{6}$. For clarity, only the 7.0 - 6.0, 4.5 - 3.5 (60x magnification), and $2.5-1.5 \mathrm{ppm}$ regions are shown. The solvent and internal standard peaks are not shown $\left(\mathrm{C}_{6} \mathrm{D}_{5} \mathrm{H}: 7.16 \mathrm{ppm} ; \mathrm{C}_{6} \mathrm{H}_{3}\left(t \mathrm{Bu}_{3}\right): 1.35 \& 7.43 \mathrm{ppm}\right)$
$(\mathbf{I})=\mathrm{Pd}(\mathrm{IMes})_{2}(\mathrm{O})_{2}(3)$
$(\mathbf{V})=(\mathbf{I V})+1 \mathrm{hr}$
(II) $=\mathbf{3}+0.4$ equiv HOAc
$(\mathbf{V I})=(\mathbf{I V})+1$ day
(III) $=\mathbf{3}+1.0$ equiv HOAc
$(\mathbf{V I I})=(\mathbf{I V})+3$ days
$(\mathbf{I V})=\mathbf{3}+2.5$ equiv. HOAc

The hydroperoxide resonance broadens into the baseline in the presence of excess HOAc (spectra IV-VII). Hydrogen bonding to excess HOAc alters the peak positions of certain resonances of the palladium complexes (e.g., compare spectra III and IV). 
Preparation of $\mathrm{Pd}(\mathrm{IMes})_{2}(\mathrm{OOH})(\mathrm{OAc})$ (4). As a representative experiment, a flask equipped with a magnetic stir bar was charged with $\mathrm{Pd}(\mathrm{IMes})_{2}\left(\mathrm{O}_{2}\right)(36.0 \mathrm{mg}, 48.2 \mu \mathrm{mol})$. Toluene $(4 \mathrm{~mL})$ was added, and the flask was sealed with a septum and removed from the glove box. An HOAc solution $(0.733 \mathrm{M})$ was prepared by diluting HOAc $(42.0 \mu \mathrm{L})$ in toluene $(1 \mathrm{~mL})$. At this point, the lights in the lab were turned off because the product, $\mathbf{4}$, appears to be somewhat light sensitive. With stirring, HOAc solution (100 $\mu \mathrm{L}, 73.4 \mu \mathrm{mol})$ was added to the palladium solution and an immediate color change occured from brown to pale yellow. The solution was stirred for 5 minutes, filtered through a plug of celite, and the solvent was removed under vacuum without heating. An additional $4 \mathrm{~mL}$ of toluene was added and removed under vacuum to ensure removal of any excess HOAc. The product, $\mathrm{Pd}(\mathrm{IMes})_{2}(\mathrm{OOH})(\mathrm{OAc})(3)$ was obtained as an off-white solid (65\% crude yield). The solid was purified further by extracting with pentane, filtering through celite, and removing the pentane under vacuum.

Analytical Data:

${ }^{1} \mathrm{H} \mathrm{NMR}\left(300 \mathrm{MHz}, \mathrm{C}_{6} \mathrm{D}_{6}\right): \delta 6.91(\mathrm{~s}, 8 \mathrm{H}), 6.13(\mathrm{~s}, 4 \mathrm{H}), 3.87(\mathrm{~s}, 1 \mathrm{H}), 2.31(\mathrm{~s}, 12 \mathrm{H}), 2.21(\mathrm{~s}$, $24 \mathrm{H}), 1.70(\mathrm{~s}, 3 \mathrm{H})$

${ }^{13} \mathrm{C}$ NMR $\left(75 \mathrm{MHz}, \mathrm{C}_{6} \mathrm{D}_{6}\right): \delta 19.1(o-\mathrm{Me}), 21.6(p-\mathrm{Me}), 24.1\left(\mathrm{CH}_{3} \mathrm{COO}-\right), 121.8(-\mathrm{HC}=\mathbf{C H}-)$, $129.4,137.2,137.4,137.7$ (aryl C), $173.8\left(\mathrm{CH}_{3} \mathrm{COO}-\right), 178.1(\mathrm{~N}-\mathrm{C}-\mathrm{N})$

HMBC NMR Correlations $(\delta \mathrm{H} / \delta \mathrm{C}): 6.13 / 178.1,1.70 / 173.8$

$\underline{\text { IR (ATR) }} v_{\max }\left(\mathrm{cm}^{-1}\right): 3503(\mathrm{~s}, \mathrm{O}-\mathrm{H}), 1626(\mathrm{~s}, \mathrm{C}=\mathrm{O}), 1488,1407,1365,1321,1274,1233,1037$, 929, 849, 733, 708, 669

MS (ESI-TOF) $m / z$ (relative intensity): 807.2(3.4) $[\mathrm{M}+\mathrm{H}]^{+}, 788.1(4.5)\left[\mathrm{M}-\mathrm{H}_{2} \mathrm{O}\right]^{+}, 773.1(7.2)$ $[\mathrm{M}-\mathrm{OOH}]^{+}, 747.1(12.4)[\mathrm{M}-\mathrm{OAc}]^{+}, 713.1(100)\left[\mathrm{M}-\mathrm{H}_{2} \mathrm{O}_{2}-\mathrm{OAc}\right]^{+}$, 305.1(47.7) [IMesH] ${ }^{+}$. Elemental Analysis Calcd for $\mathrm{C}_{44} \mathrm{H}_{52} \mathrm{~N}_{4} \mathrm{O}_{4} \mathrm{Pd}(\%)$ : C 65.46, H 6.49, N 6.94. Found (average of two measurements): C 65.64, H 6.50, N 6.48. 


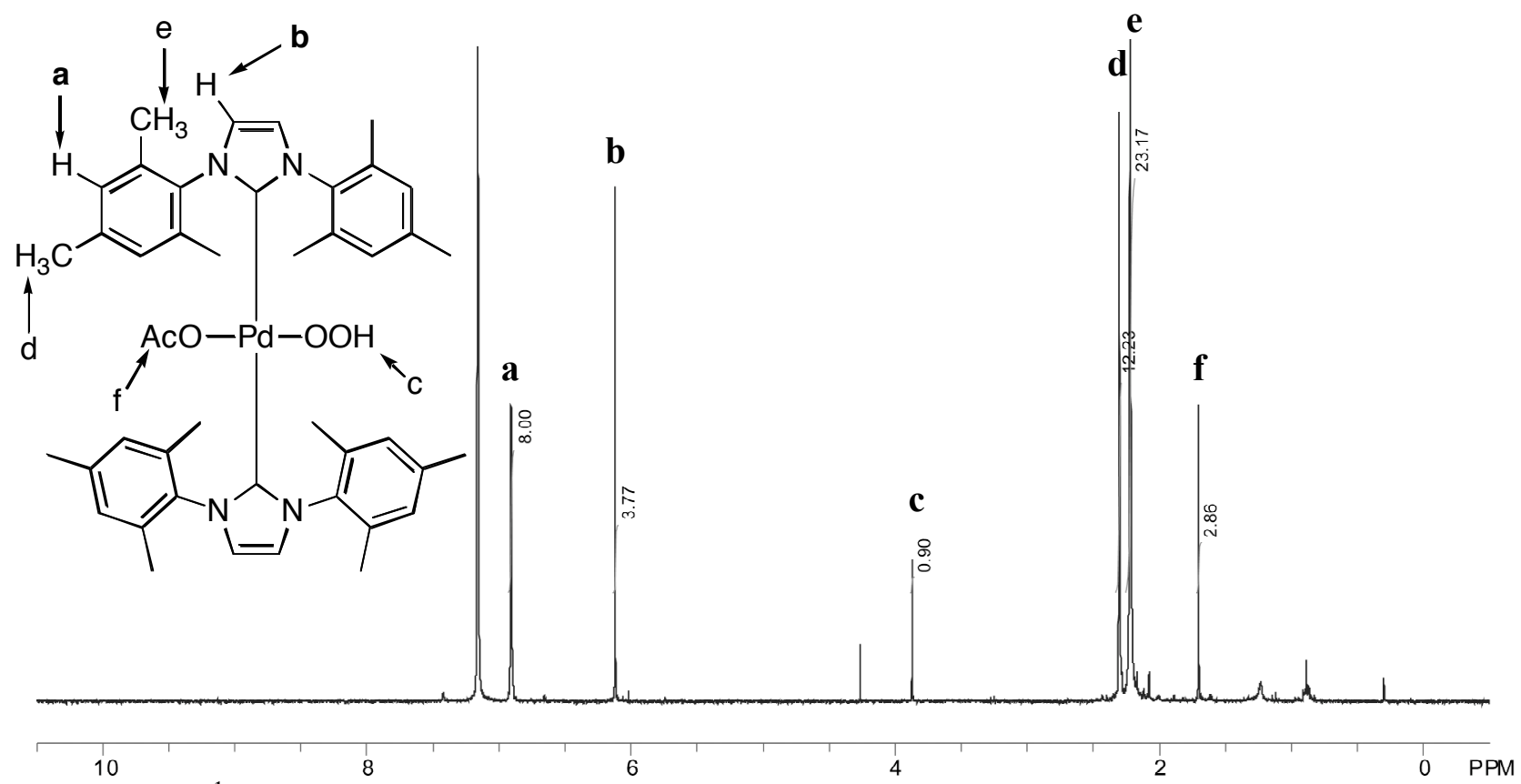

Figure S6. ${ }^{1} \mathrm{H}-\mathrm{NMR}$ spectrum of (4). (Note: peak at $4.27 \mathrm{ppm}$ corresponds to residual $\mathrm{CH}_{2} \mathrm{Cl}_{2}$.) 


\section{Preparation of Pd(IMes) $)_{2}\left({ }^{18} \mathrm{O}^{18} \mathrm{OH}\right)(\mathrm{OAc})\left({ }^{18}{ }^{18} \mathrm{O}_{2}\right)$ and $\mathrm{Pd}(\mathrm{IMes})_{2}(\mathrm{OOD})(\mathrm{OAc})$ (4-}

$\left.\boldsymbol{d}_{1}\right)$. The procedure for the preparation of $\left(\mathbf{4}^{\mathbf{1 8}} \mathbf{O}_{2}\right)$ and $\left(\mathbf{4 - d _ { 1 } )}\right.$ was identical to that for 4 except that $\mathrm{Pd}(\mathrm{IMes})_{2}\left({ }^{18} \mathrm{O}_{2}\right)$ was used in the synthesis of $\left(\mathbf{4 -}^{\mathbf{1 8}} \mathbf{O}_{2}\right)$ and DOAc was used in the synthesis of (4-d $\left.d_{1}\right)$.

Analytical Data $\left(\mathbf{4 -}^{18} \mathbf{O}_{2}\right)$ :

IR (ATR): identical to 3 except minus 3503 and plus 3491; MS (ESI-TOF) $\mathrm{m} / \mathrm{z}$ (relative intensity): 811.1(0.004) $[\mathrm{M}+\mathrm{H}]^{+}, 773.1(0.5)\left[\mathrm{M}-{ }^{18} \mathrm{O}^{18} \mathrm{OH}\right]^{+}, 751.1(0.07)[\mathrm{M}-\mathrm{OAc}]^{+}, 713.1(100)$ $\left[\mathrm{M}-\mathrm{H}_{2}{ }^{18} \mathrm{O}_{2} / \mathrm{OAc}\right]^{+}, 305.1(2.5)[\mathrm{IMesH}]^{+}$.

Analytical Data (4-d $\boldsymbol{d}_{\mathbf{1}}$ :

IR (ATR): identical to 4 except plus 2591

Infrared spectroscopic data for $4, \mathbf{4}^{\mathbf{1 8}} \mathrm{O}_{\mathbf{2}}$, and 4- $\boldsymbol{d}_{\mathbf{1}}$ and each difference spectrum is shown in figure S7. Mass spectra for $\mathbf{4}$ and $\mathbf{4 -}^{\mathbf{1 8}} \mathbf{O}_{\mathbf{2}}$ are shown in Figures S8 and S9.

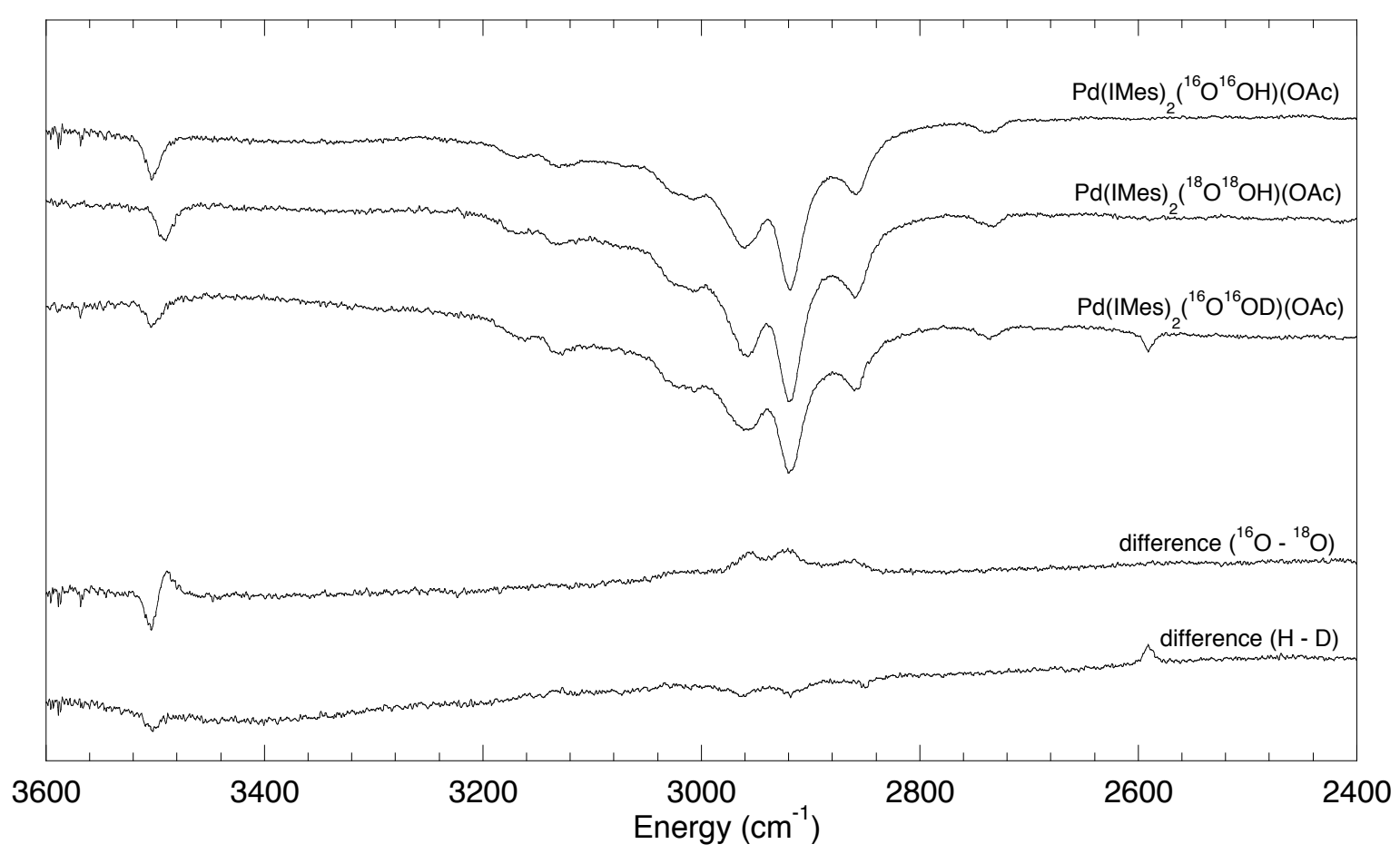

Figure S7. FTIR spectra of 4, 4- ${ }^{\mathbf{1 8}} \mathbf{O}_{\mathbf{2}}$, and 4- $\boldsymbol{d}_{\mathbf{1}}$ in the $3600-2400 \mathrm{~cm}^{-1}$ region and their difference spectra. 


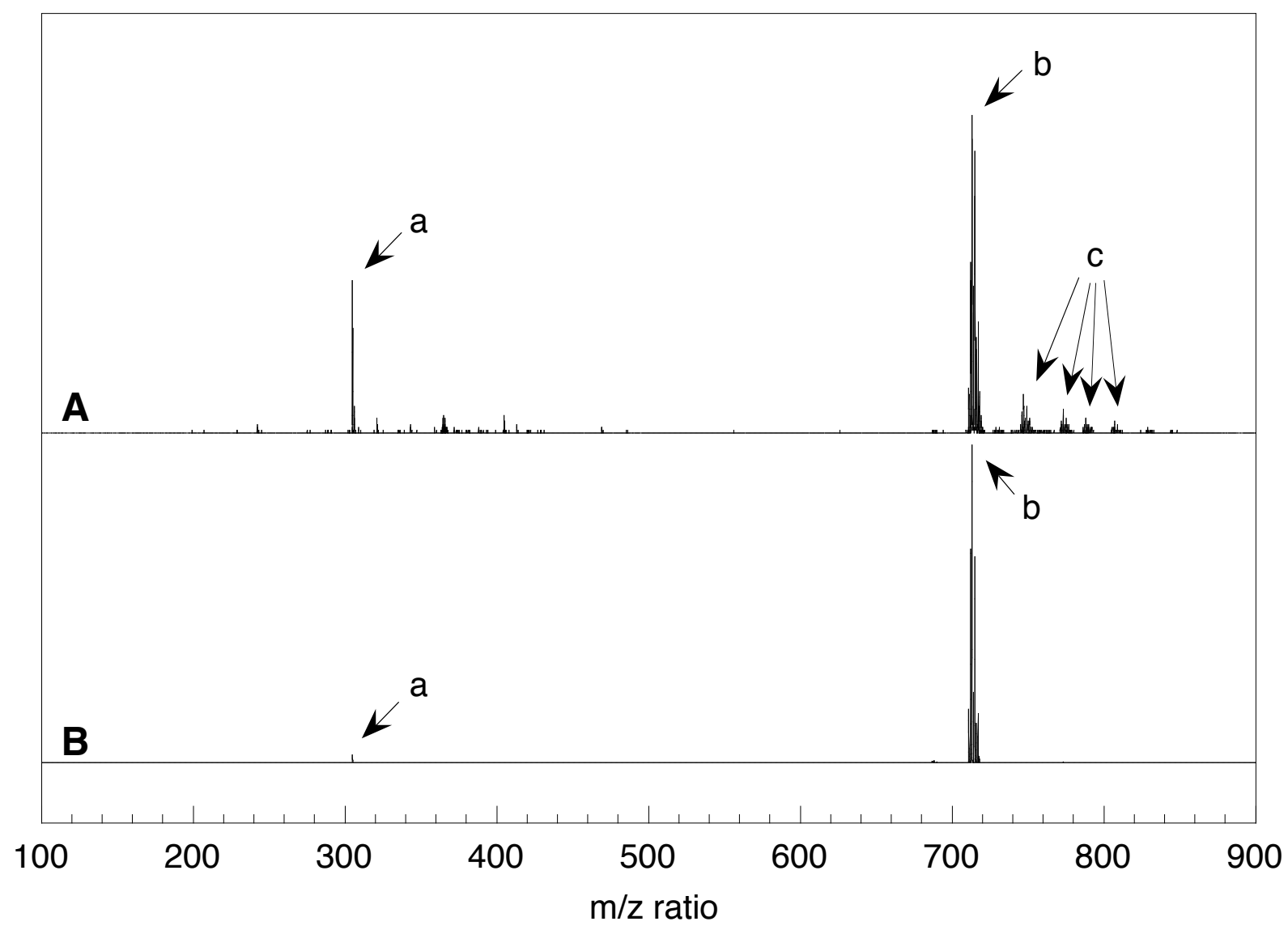

Figure S8. Full ESI-TOF mass spectra of 4 (A) and $4-{ }^{18} \mathbf{O}_{\mathbf{2}}(\mathbf{B})$. The peak (a) corresponds to the free imidazolium cation. The peak $(\mathbf{b})$ at $\mathrm{m} / \mathrm{z}=713.1$, corresponding to the net loss of hydrogen peroxide and acetate anion from $\mathbf{4}$, is the predominant mass fragment in both (A) and (B). The molecular ion and other less intense mass fragments (c) for $\mathbf{4}$ and $\mathbf{4 -}^{\mathbf{1 8}} \mathrm{O}_{2}$ are shown in Figure S9. 


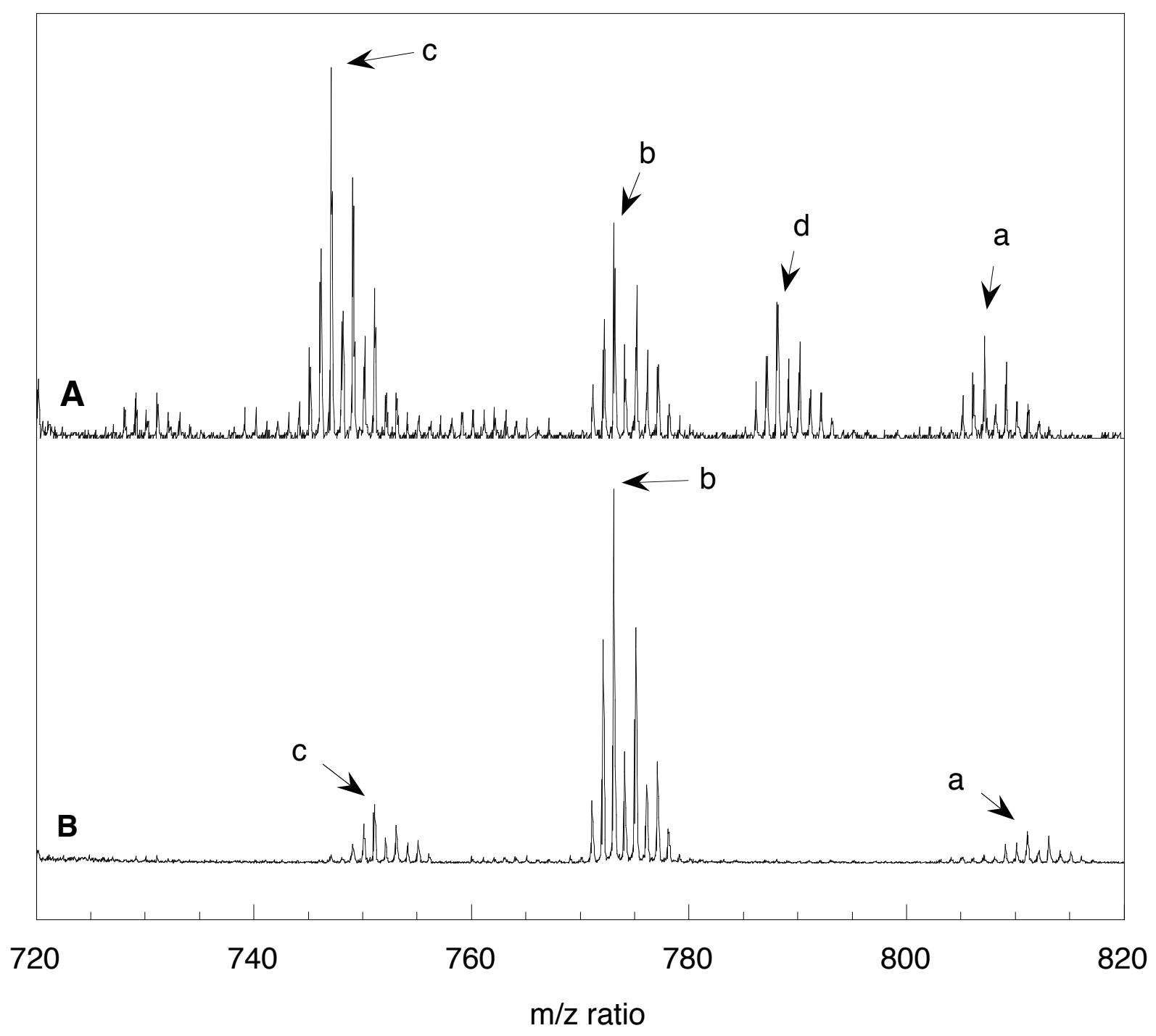

Figure S9. ESI-TOF mass spectra of $\mathbf{4}(\mathbf{A})$ and $\mathbf{4}^{-18} \mathbf{O}_{2}(\mathbf{B})$ in the region $\mathrm{m} / \mathrm{z}=720-820$. The $[\mathrm{M}+\mathrm{H}]^{+}(\mathbf{a})$ and $[\mathrm{M}-\mathrm{OAc}]^{+}$(c) peaks both shift up by four mass units for ${ }^{18} \mathrm{O}$-labeled complex, while the $[\mathrm{M}-\mathrm{OOH}]^{+}$peak (b) remains unchanged. The mass fragment (d) was only observed for 4 and reflects the net loss of $\mathrm{HOH}$ from the molecular ion of 4 . The absence of (d) for $4-^{18} \mathrm{O}$ and differences in peak intensities for (A) and (B) arises from concentration differences in the solutions used to obtain the mass spectra. 
Independent Synthesis of Pd(IMes) $)_{2}(\mathbf{O A c})_{2}$ (5). In a 25mL flask, $\mathrm{Pd}(\mathrm{OAc})_{2}(0.101 \mathrm{~g}$, $0.45 \mathrm{mmol})$ was dissolved in dry THF $(8 \mathrm{~mL})$. A solution of IMes $(0.290 \mathrm{~g}, 0.95 \mathrm{mmol})$ in THF $(5 \mathrm{~mL})$ was added to the stirred $\mathrm{Pd}(\mathrm{OAc})_{2}$ solution. After $13 \mathrm{hrs}$, the reaction solution was poured into $70 \mathrm{~mL}$ of hexanes and placed in a $-30{ }^{\circ} \mathrm{C}$ freezer for $1 \mathrm{hr}$. The off-white solid product was obtained by filtration (65\% yield). X-ray quality crystals were grown by slow diffusion of hexane into a saturated $\mathrm{CH}_{2} \mathrm{Cl}_{2}$ solution.

Analytical Data:

$\underline{{ }^{1} \mathrm{H} \mathrm{NMR}}\left(300 \mathrm{MHz}, \mathrm{C}_{6} \mathrm{D}_{6}\right): \delta 6.91(\mathrm{~s}, 8 \mathrm{H}), 6.05(\mathrm{~s}, 4 \mathrm{H}), 2.35(\mathrm{~s}, 12 \mathrm{H}), 2.21(\mathrm{~s}, 24 \mathrm{H}), 1.60(\mathrm{~s}$, $6 \mathrm{H}) .{ }^{13} \mathrm{C} \mathrm{NMR}\left(75 \mathrm{MHz}, \mathrm{C}_{6} \mathrm{D}_{6}\right): \delta 19.4(o-\mathrm{Me}), 21.6(p-\mathrm{Me}), 23.7\left(\mathrm{CH}_{3} \mathrm{COO}-\right), 122.4(-$ $\mathrm{HC}=\mathbf{C H}-), 129.6,136.7,137.1,137.6(\operatorname{aryl} \mathrm{C}), 171.7(\mathrm{~N}-\mathbf{C}-\mathrm{N}), 174.2\left(\mathrm{CH}_{3} \mathbf{C O O}-\right)$; HMBC NMR Correlations $(\delta \mathrm{H} / \delta \mathrm{C}): 6.05 / 171.7,1.60 / 174.2$; X-ray: (see data and tables below)
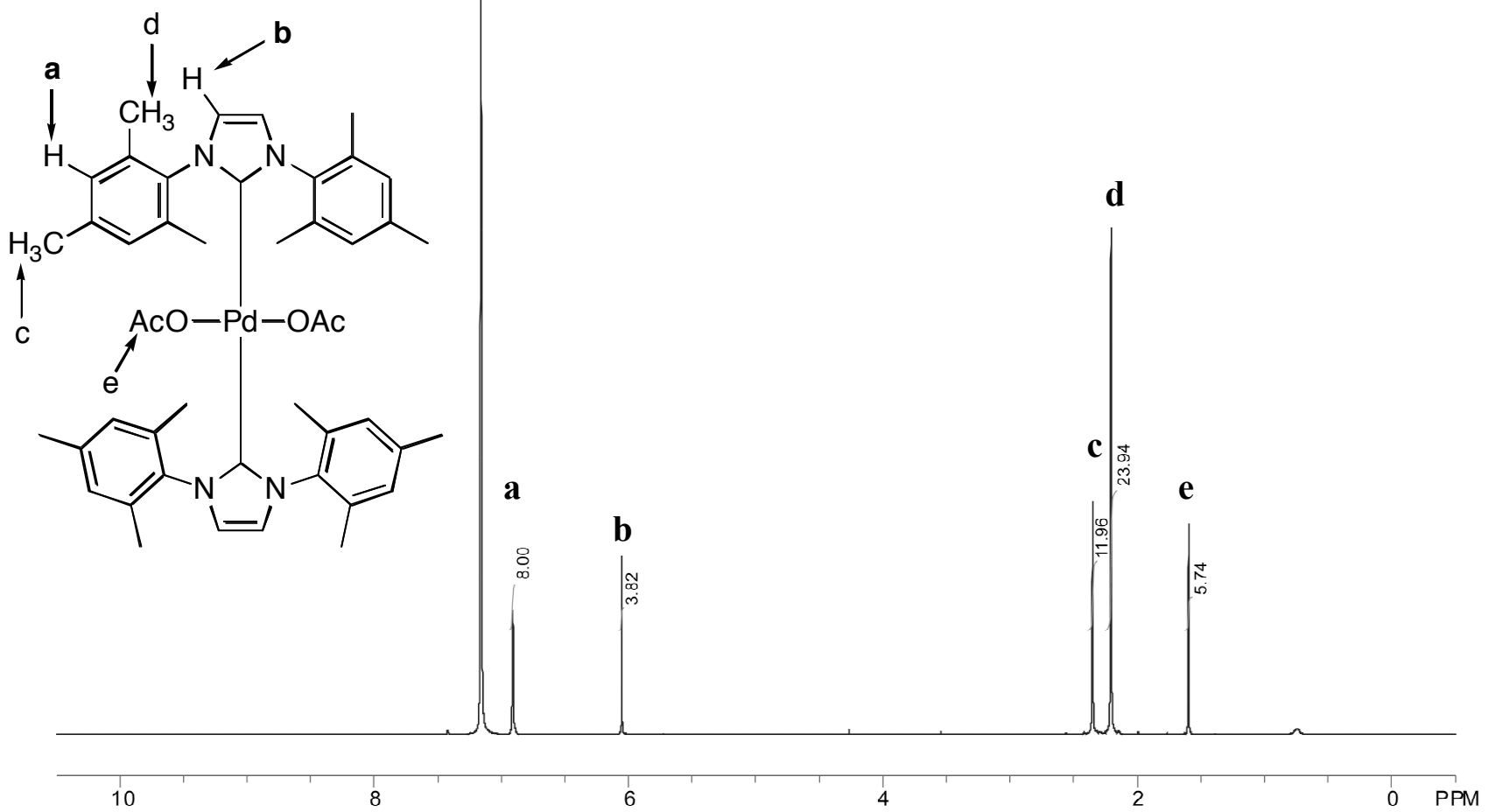

Figure S10. ${ }^{1} \mathrm{H}-\mathrm{NMR}$ spectrum of $\mathbf{5}$. 

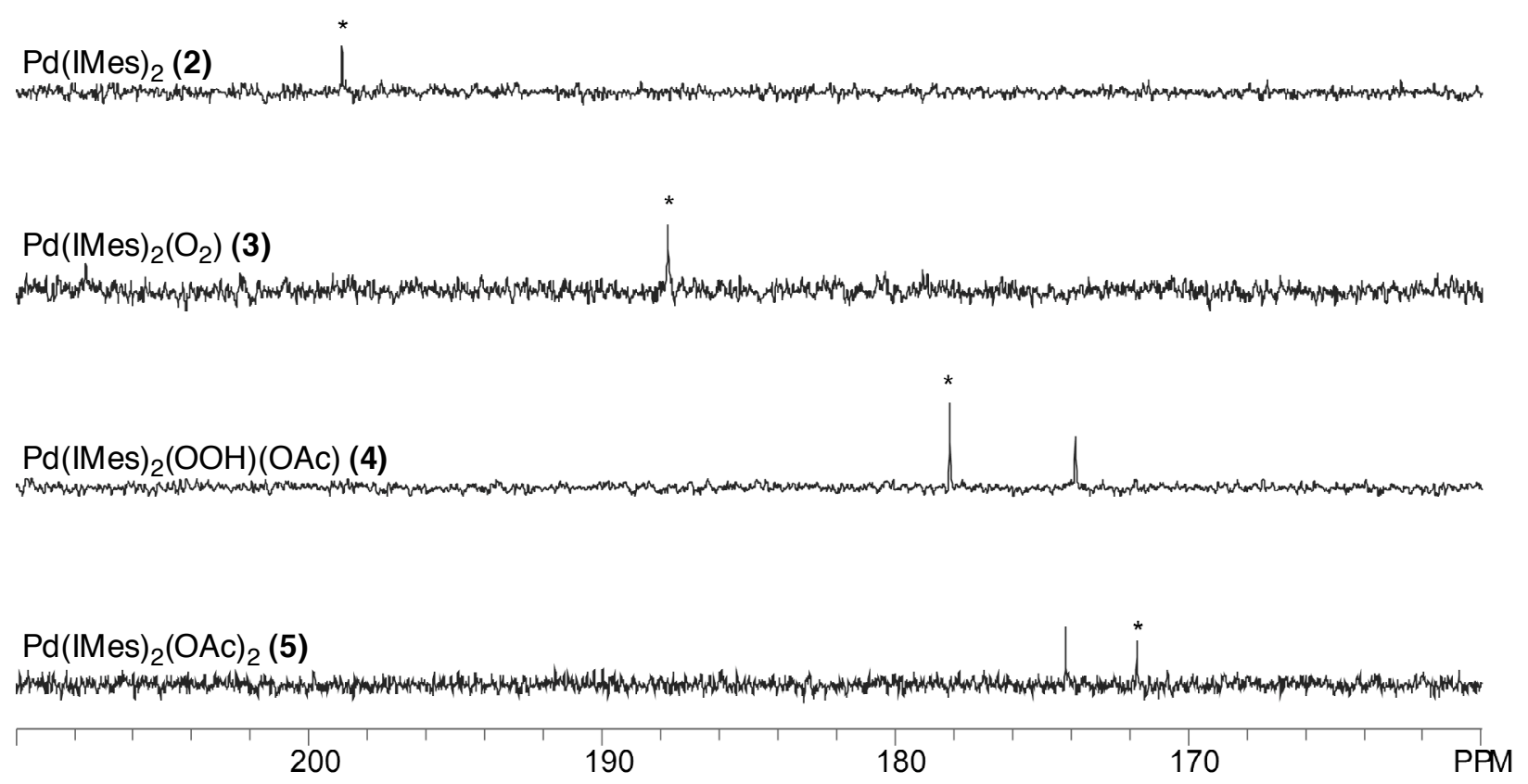

Figure S11. Stack plot of ${ }^{13} \mathrm{C}-\mathrm{NMR}$ spectra of (2), (3), (4), and (5) in the downfield carbene region. All carbene carbon resonances are marked with an asterisk.

Detection of Hydrogen Peroxide. Literature procedures were utilized in the spectrophotometric detection of $\mathrm{H}_{2} \mathrm{O}_{2}$ as a peroxotitanyl species. ${ }^{2}$ Calibration curves were constructed for solutions of $\mathrm{Pd}(\mathrm{IMes})_{2}\left(\mathrm{O}_{2}\right), \mathbf{3}$, and for $\mathrm{H}_{2} \mathrm{O}_{2} / \mathrm{Ti}\left(\mathrm{OBu}^{\mathrm{n}}\right)_{4}$ in ethanol. In the former case, standardized solutions of 3 in ethanol were prepared containing $\mathrm{H}_{2} \mathrm{SO}_{4}(0.72 \mathrm{M})$ and different concentrations of $3(0,0.42,0.88,1.06,1.83$, and $2.32 \mathrm{mM})$. A plot of $\mathrm{Abs}_{410 \mathrm{~nm}}$ versus [Pd] yielded a slope of $0.2155 \mathrm{mM}^{-1}$. Standardized $\mathrm{H}_{2} \mathrm{O}_{2} / \mathrm{Ti}\left(\mathrm{OBu}^{\mathrm{n}}\right)_{4}$ solutions in ethanol were prepared containing $\mathrm{H}_{2} \mathrm{SO}_{4}(0.72 \mathrm{M}), \mathrm{Ti}\left(\mathrm{OBu}^{\mathrm{n}}\right)_{4}(15.9 \mathrm{mM})$ and different concentrations of $\mathrm{H}_{2} \mathrm{O}_{2}(0,0.84,1.69,2.65,3.73,4.74 \mathrm{mM})$. A plot of $\mathrm{Abs}_{410 \mathrm{~nm}}$ versus $\left[\mathrm{H}_{2} \mathrm{O}_{2}\right]$ yielded a slope of $0.7263 \mathrm{mM}^{-1}$. Experimental determination of $\mathrm{H}_{2} \mathrm{O}_{2}$ was effected as follows: A $10 \mathrm{~mL}$ volumetric flask was charged with $\mathrm{Pd}(\mathrm{IMes})_{2}\left(\mathrm{O}_{2}\right)(13.9 \mathrm{mg}, 18.6 \mu \mathrm{mol})$, fitted with a septum and removed from the glove box. The volumetric flask was partially filled with an ethanol solution of $\mathrm{Ti}\left(\mathrm{OBu}^{\mathrm{n}}\right)_{4} / \mathrm{H}_{2} \mathrm{SO}_{4}(0.72 \mathrm{M} / 15.9 \mathrm{mM})$, mixed on a vortexer for 4 minutes, and filled to the $10 \mathrm{~mL}$ line on the volumetric with the $\mathrm{Ti}\left(\mathrm{OBu}^{\mathrm{n}}\right)_{4} / \mathrm{H}_{2} \mathrm{SO}_{4}$ solution to yield a $1.86 \mathrm{mM}$ palladium solution. The solution absorbance was obtained in triplicate with independent aliquots of the solution.

\footnotetext{
2 (a) Eisenberg, G. M. Ind. Eng. Chem. Anal. Ed. 1943, 15, 327-328. (b) Werner, W. U.S. Patent 4,908,323, 1990.
} 
Absorbance readings of 1.351, 1.353, 1.347 at $410 \mathrm{~nm}$ yield an average of 1.350 A.U. Based on the calibration curves $\left(\mathrm{Abs}_{\text {total }}=0.7263\left[\mathrm{H}_{2} \mathrm{O}_{2}\right]+0.2155[\mathrm{Pd}]\right)$, this absorbance reading corresponds to $\left[\mathrm{H}_{2} \mathrm{O}_{2}\right]=1.31 \mathrm{mM}(70 \%$ yield $)$. The fate of the palladium complex under the conditions of the colorimetric assay was investigated by titrating $\mathrm{H}_{2} \mathrm{SO}_{4}$ against $\mathrm{Pd}(\mathrm{IMes})_{2}\left(\mathrm{O}_{2}\right)$ (3) in methanol- $d_{4}$ and monitoring the reaction by ${ }^{1} \mathrm{H}$ NMR spectroscopy. Sequential formation of two new bis-carbene species is qualitatively consistent with the formation of singly protonated and doubly protonated species analogous to $\mathbf{4}$ and $\mathbf{5}$. Detailed characterization of these entities was not pursued. 


\section{X-ray crystallographic Analysis of Pd(IMes)2, (2).}

A red air-sensitive crystal with approximate dimensions $0.43 \times 0.32 \times 0.26 \mathrm{~mm}^{3}$ was selected under oil under ambient conditions and attached to the tip of a nylon loop. The crystal was mounted in a stream of cold nitrogen at $100(2) \mathrm{K}$ and centered in the X-ray beam by using a video camera.

The crystal evaluation and data collection were performed on a Bruker CCD-1000 diffractometer with $\mathrm{Mo}_{\alpha}(\lambda=0.71073 \AA)$ radiation and the diffractometer to crystal distance of $4.9 \mathrm{~cm}$.

The initial cell constants were obtained from three series of $\omega$ scans at different starting angles. Each series consisted of 20 frames collected at intervals of $0.3^{\circ}$ in a $6^{\circ}$ range about $\omega$ with the exposure time of 20 seconds per frame. A total of 243 reflections was obtained. The reflections were successfully indexed by an automated indexing routine built in the SMART program. The final cell constants were calculated from a set of 21079 strong reflections from the actual data collection.

The data were collected by using the hemisphere data collection routine. The reciprocal space was surveyed to the extent of a full sphere to a resolution of $0.80 \AA$. A total of 29842 data were harvested by collecting three sets of frames with $0.3^{\circ}$ scans in $\omega$ with an exposure time 80 sec per frame. These highly redundant datasets were corrected for Lorentz and polarization effects. The absorption correction was based on fitting a function to the empirical transmission surface as sampled by multiple equivalent measurements ${ }^{3}$.

\section{Structure Solution and Refinement}

The systematic absences in the diffraction data were uniquely consistent for the space group $P 2{ }_{1} / c$ that yielded chemically reasonable and computationally stable results of refinement ${ }^{3}$.

A successful solution by the direct methods provided most non-hydrogen atoms from the $E$-map. The remaining non-hydrogen atoms were located in an alternating series of least-squares cycles and difference Fourier maps. All non-hydrogen atoms were refined with anisotropic displacement coefficients. All hydrogen atoms were included in the structure factor calculation

\footnotetext{
${ }^{3}$ Bruker-AXS. (2000-2001) SADABS V.2.03, SAINT V.6.22, SHELXTL V.6.10

\& SMART 5.622 Software Reference Manuals. Bruker-AXS, Madison, Wisconsin, USA.
} 
at idealized positions and were allowed to ride on the neighboring atoms with relative isotropic displacement coefficients.

The final least-squares refinement of 436 parameters against 7594 data resulted in residuals $R$ (based on $F^{2}$ for $I \geq 2 \sigma$ ) and $w R$ (based on $F^{2}$ for all data) of 0.0469 and 0.1110 , respectively. The final difference Fourier map was featureless.

The ORTEP diagram is drawn with $50 \%$ probability ellipsoids. 


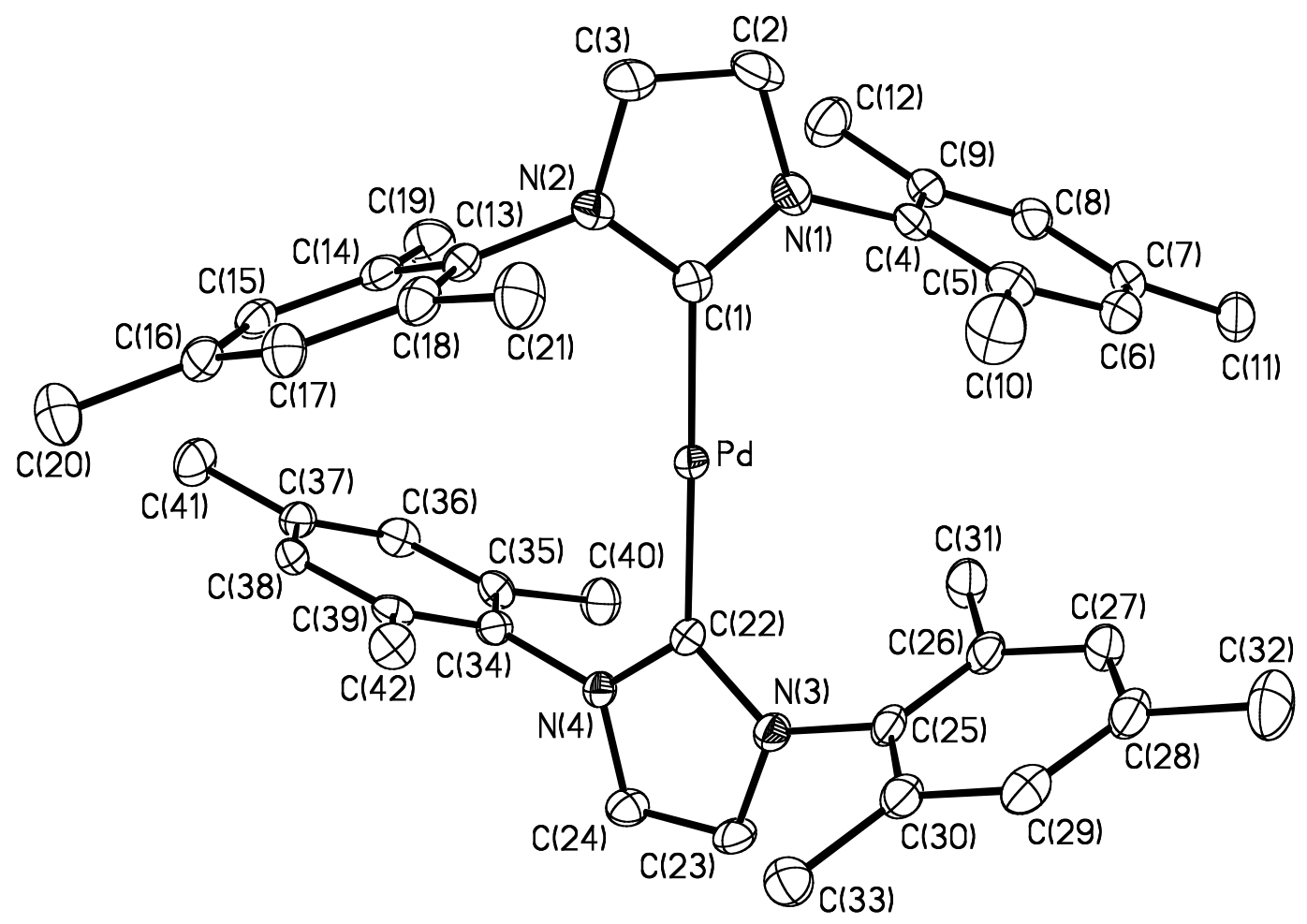

Figure S12. A molecular drawing of (2) with 50\% probability ellipsoids. 
Table S2. Crystal data and structure refinement for (2).

\begin{tabular}{|c|c|c|}
\hline Identification code & \multicolumn{2}{|l|}{ sta11 } \\
\hline Empirical formula & \multicolumn{2}{|l|}{$\mathrm{C}_{42} \mathrm{H}_{48} \mathrm{~N}_{4} \mathrm{Pd}$} \\
\hline Formula weight & \multicolumn{2}{|l|}{715.24} \\
\hline Temperature & \multicolumn{2}{|l|}{$100(2) \mathrm{K}$} \\
\hline Wavelength & \multicolumn{2}{|l|}{$0.71073 \AA$} \\
\hline Crystal system & \multicolumn{2}{|l|}{ Monoclinic } \\
\hline Space group & \multicolumn{2}{|l|}{$\mathrm{P} 2(1) / \mathrm{c}$} \\
\hline \multirow[t]{3}{*}{ Unit cell dimensions } & $\mathrm{a}=15.6449(15) \AA$ & $\alpha=90^{\circ}$. \\
\hline & $\mathrm{b}=16.4566(16) \AA$ & $\beta=98.65$ \\
\hline & $\mathrm{c}=14.5862(14) \AA$ & $\gamma=90^{\circ}$. \\
\hline Volume & \multicolumn{2}{|l|}{$3712.6(6) \AA^{3}$} \\
\hline $\mathrm{Z}$ & \multicolumn{2}{|l|}{4} \\
\hline Density (calculated) & \multicolumn{2}{|l|}{$1.280 \mathrm{Mg} / \mathrm{m}^{3}$} \\
\hline Absorption coefficient & \multicolumn{2}{|l|}{$0.533 \mathrm{~mm}^{-1}$} \\
\hline $\mathrm{F}(000)$ & \multicolumn{2}{|l|}{1496} \\
\hline Crystal size & \multicolumn{2}{|c|}{$0.43 \times 0.32 \times 0.26 \mathrm{~mm}^{3}$} \\
\hline Theta range for data collection & \multicolumn{2}{|l|}{1.81 to $26.40^{\circ}$. } \\
\hline Index ranges & \multicolumn{2}{|c|}{$-19<=\mathrm{h}<=19,-20<=\mathrm{k}<=20,-17<=1<=18$} \\
\hline Reflections collected & \multicolumn{2}{|l|}{29842} \\
\hline Independent reflections & \multicolumn{2}{|c|}{$7594[\mathrm{R}(\mathrm{int})=0.0358]$} \\
\hline Completeness to theta $=26.40^{\circ}$ & \multicolumn{2}{|l|}{$99.6 \%$} \\
\hline Absorption correction & \multicolumn{2}{|c|}{ Empirical with SADABS } \\
\hline Max. and min. transmission & \multicolumn{2}{|l|}{0.8738 and 0.8031} \\
\hline Refinement method & \multicolumn{2}{|c|}{ Full-matrix least-squares on $\mathrm{F}^{2}$} \\
\hline Data / restraints / parameters & \multicolumn{2}{|l|}{7594 / 0 / 436} \\
\hline Goodness-of-fit on $\mathrm{F}^{2}$ & \multicolumn{2}{|l|}{1.200} \\
\hline Final $\mathrm{R}$ indices $[\mathrm{I}>2 \operatorname{sigma}(\mathrm{I})]$ & \multicolumn{2}{|c|}{$\mathrm{R} 1=0.0469, \mathrm{wR} 2=0.1092$} \\
\hline $\mathrm{R}$ indices (all data) & \multicolumn{2}{|c|}{$\mathrm{R} 1=0.0518, \mathrm{wR} 2=0.1110$} \\
\hline Largest diff. peak and hole & \multicolumn{2}{|c|}{0.822 and -0.674 e. $\AA^{-3}$} \\
\hline
\end{tabular}


Table S3. Atomic coordinates ( $\left.\mathrm{x} 10^{4}\right)$ and equivalent isotropic displacement parameters $\left(\AA^{2} \mathrm{x} 10^{3}\right)$ for (2). $U(e q)$ is defined as one third of the trace of the orthogonalized $U^{i j}$ tensor.

\begin{tabular}{|c|c|c|c|c|}
\hline & $\mathrm{x}$ & $\mathrm{y}$ & $\mathrm{z}$ & $\mathrm{U}(\mathrm{eq})$ \\
\hline$\overline{\mathrm{Pd}}$ & $2568(1)$ & $78(1)$ & 5902(1) & $16(1)$ \\
\hline $\mathrm{N}(1)$ & $2321(2)$ & 1829(2) & $6495(2)$ & $24(1)$ \\
\hline $\mathrm{N}(2)$ & $1676(2)$ & $1622(2)$ & $5126(2)$ & $22(1)$ \\
\hline $\mathrm{N}(3)$ & $2926(2)$ & $-1650(2)$ & $6638(2)$ & $18(1)$ \\
\hline $\mathrm{N}(4)$ & $3256(2)$ & $-1530(2)$ & $5281(2)$ & $17(1)$ \\
\hline $\mathrm{C}(1)$ & $2178(2)$ & $1230(2)$ & $5837(2)$ & $20(1)$ \\
\hline$C(2)$ & 1922(3) & 2554(2) & $6202(3)$ & $34(1)$ \\
\hline$C(3)$ & $1520(3)$ & 2423(2) & $5337(3)$ & $35(1)$ \\
\hline$C(4)$ & $2878(2)$ & 1720(2) & $7372(2)$ & $21(1)$ \\
\hline$C(5)$ & $2544(2)$ & 1362(2) & $8108(3)$ & $25(1)$ \\
\hline$C(6)$ & $3101(2)$ & $1247(2)$ & $8934(2)$ & $25(1)$ \\
\hline$C(7)$ & $3966(2)$ & $1465(2)$ & 9031(2) & $22(1)$ \\
\hline$C(8)$ & $4266(2)$ & 1837(2) & $8288(2)$ & $23(1)$ \\
\hline $\mathrm{C}(9)$ & $3733(2)$ & 1976(2) & $7454(2)$ & $22(1)$ \\
\hline$C(10)$ & 1618(3) & 1080(3) & 7998(3) & $40(1)$ \\
\hline$C(11)$ & $4572(2)$ & 1282(2) & $9913(2)$ & $28(1)$ \\
\hline$C(12)$ & $4070(3)$ & 2391(2) & 6661(3) & $33(1)$ \\
\hline$C(13)$ & $1334(2)$ & $1236(2)$ & $4259(2)$ & $20(1)$ \\
\hline$C(14)$ & 1871(2) & 1137(2) & $3583(2)$ & 19(1) \\
\hline$C(15)$ & $1505(2)$ & $788(2)$ & $2746(2)$ & 19(1) \\
\hline$C(16)$ & $647(2)$ & $534(2)$ & $2578(2)$ & $21(1)$ \\
\hline$C(17)$ & $145(2)$ & $637(2)$ & $3274(2)$ & $22(1)$ \\
\hline$C(18)$ & $472(2)$ & $985(2)$ & $4121(2)$ & $21(1)$ \\
\hline C(19) & $2816(2)$ & $1360(2)$ & $3763(3)$ & $29(1)$ \\
\hline$C(20)$ & 291(2) & 141(2) & $1665(3)$ & $30(1)$ \\
\hline$C(21)$ & $-76(3)$ & 1063(3) & $4882(3)$ & $30(1)$ \\
\hline$C(22)$ & $2937(2)$ & $-1085(2)$ & $5949(2)$ & $17(1)$ \\
\hline$C(23)$ & $3230(2)$ & $-2405(2)$ & $6405(2)$ & $22(1)$ \\
\hline$C(24)$ & $3435(2)$ & $-2329(2)$ & $5550(2)$ & $21(1)$ \\
\hline$C(25)$ & $2597(2)$ & $-1485(2)$ & 7494(2) & 19(1) \\
\hline$C(26)$ & $3093(2)$ & $-1012(2)$ & $8163(2)$ & $21(1)$ \\
\hline$C(27)$ & $2768(2)$ & $-872(2)$ & $8992(2)$ & $25(1)$ \\
\hline$C(28)$ & 1972(2) & $-1183(2)$ & 9141(2) & $25(1)$ \\
\hline$C(29)$ & $1502(2)$ & $-1647(2)$ & $8452(2)$ & $24(1)$ \\
\hline$C(30)$ & $1807(2)$ & $-1814(2)$ & $7619(2)$ & $22(1)$ \\
\hline$C(31)$ & $3933(2)$ & $-639(2)$ & $8006(2)$ & $26(1)$ \\
\hline$C(32)$ & $1635(3)$ & $-1009(3)$ & $10039(3)$ & $36(1)$ \\
\hline C(33) & $1283(2)$ & $-2327(2)$ & 6889(3) & $25(1)$ \\
\hline$C(34)$ & $3410(2)$ & $-1204(2)$ & $4402(2)$ & $17(1)$ \\
\hline$C(35)$ & $4256(2)$ & $-1024(2)$ & $4278(2)$ & 19(1) \\
\hline C(36) & $4392(2)$ & $-733(2)$ & $3413(2)$ & 21(1) \\
\hline$C(37)$ & $3713(2)$ & $-622(2)$ & 2694(2) & $20(1)$ \\
\hline C(38) & $2879(2)$ & $-806(2)$ & $2847(2)$ & $18(1)$ \\
\hline C(39) & $2706(2)$ & $-1088(2)$ & $3704(2)$ & $17(1)$ \\
\hline $\mathrm{C}(40)$ & $4995(2)$ & $-1104(2)$ & $5062(3)$ & $26(1)$ \\
\hline C(41) & $3869(3)$ & $-296(2)$ & $1765(3)$ & $29(1)$ \\
\hline C(42) & 1793(2) & $-1215(2)$ & $3876(2)$ & $22(1)$ \\
\hline
\end{tabular}


Table S4. Bond lengths $[\AA]$ and angles $\left[{ }^{\circ}\right]$ for (2).

\begin{tabular}{|c|c|c|c|}
\hline$\overline{\mathrm{Pd}-\mathrm{C}(1)}$ & $1.990(3)$ & $\mathrm{C}(14)-\mathrm{C}(15)$ & $1.392(5)$ \\
\hline $\mathrm{Pd}-\mathrm{C}(22)$ & $1.997(3)$ & $\mathrm{C}(14)-\mathrm{C}(19)$ & $1.507(5)$ \\
\hline $\mathrm{N}(1)-\mathrm{C}(1)$ & $1.371(4)$ & $C(15)-C(16)$ & $1.391(5)$ \\
\hline $\mathrm{N}(1)-\mathrm{C}(2)$ & $1.385(5)$ & $\mathrm{C}(16)-\mathrm{C}(17)$ & $1.384(5)$ \\
\hline $\mathrm{N}(1)-\mathrm{C}(4)$ & $1.446(4)$ & $C(16)-C(20)$ & $1.509(5)$ \\
\hline $\mathrm{N}(2)-\mathrm{C}(1)$ & $1.365(4)$ & $\mathrm{C}(17)-\mathrm{C}(18)$ & $1.389(5)$ \\
\hline $\mathrm{N}(2)-\mathrm{C}(3)$ & $1.384(5)$ & $C(18)-C(21)$ & $1.507(5)$ \\
\hline $\mathrm{N}(2)-\mathrm{C}(13)$ & $1.444(4)$ & $C(23)-C(24)$ & $1.339(5)$ \\
\hline $\mathrm{N}(3)-\mathrm{C}(22)$ & $1.371(4)$ & $C(25)-C(30)$ & $1.387(5)$ \\
\hline$N(3)-C(23)$ & $1.391(4)$ & $\mathrm{C}(25)-\mathrm{C}(26)$ & $1.392(5)$ \\
\hline $\mathrm{N}(3)-\mathrm{C}(25)$ & $1.446(4)$ & $C(26)-C(27)$ & $1.400(5)$ \\
\hline $\mathrm{N}(4)-\mathrm{C}(22)$ & $1.372(4)$ & $C(26)-C(31)$ & $1.499(5)$ \\
\hline $\mathrm{N}(4)-\mathrm{C}(24)$ & $1.389(4)$ & $\mathrm{C}(27)-\mathrm{C}(28)$ & $1.393(5)$ \\
\hline $\mathrm{N}(4)-\mathrm{C}(34)$ & $1.443(4)$ & $\mathrm{C}(28)-\mathrm{C}(29)$ & $1.382(5)$ \\
\hline$C(2)-C(3)$ & $1.341(5)$ & $C(28)-C(32)$ & $1.512(5)$ \\
\hline $\mathrm{C}(4)-\mathrm{C}(9)$ & $1.391(5)$ & $\mathrm{C}(29)-\mathrm{C}(30)$ & $1.398(5)$ \\
\hline$C(4)-C(5)$ & $1.393(5)$ & $\mathrm{C}(30)-\mathrm{C}(33)$ & $1.502(5)$ \\
\hline $\mathrm{C}(5)-\mathrm{C}(6)$ & $1.390(5)$ & $\mathrm{C}(34)-\mathrm{C}(35)$ & $1.394(5)$ \\
\hline$C(5)-C(10)$ & $1.507(5)$ & $C(34)-C(39)$ & $1.396(5)$ \\
\hline$C(6)-C(7)$ & $1.386(5)$ & $\mathrm{C}(35)-\mathrm{C}(36)$ & $1.396(5)$ \\
\hline $\mathrm{C}(7)-\mathrm{C}(8)$ & $1.387(5)$ & $\mathrm{C}(35)-\mathrm{C}(40)$ & $1.504(5)$ \\
\hline$C(7)-C(11)$ & $1.509(5)$ & $\mathrm{C}(36)-\mathrm{C}(37)$ & $1.388(5)$ \\
\hline $\mathrm{C}(8)-\mathrm{C}(9)$ & $1.386(5)$ & $\mathrm{C}(37)-\mathrm{C}(38)$ & $1.389(5)$ \\
\hline $\mathrm{C}(9)-\mathrm{C}(12)$ & $1.506(5)$ & $\mathrm{C}(37)-\mathrm{C}(41)$ & $1.511(5)$ \\
\hline $\mathrm{C}(13)-\mathrm{C}(18)$ & $1.396(5)$ & $\mathrm{C}(38)-\mathrm{C}(39)$ & $1.398(5)$ \\
\hline C(13)-C(14) & $1.397(5)$ & $\mathrm{C}(39)-\mathrm{C}(42)$ & $1.502(4)$ \\
\hline $\mathrm{C}(1)-\mathrm{Pd}-\mathrm{C}(22)$ & $178.80(13)$ & $\mathrm{C}(8)-\mathrm{C}(9)-\mathrm{C}(4)$ & $118.0(3)$ \\
\hline $\mathrm{C}(1)-\mathrm{N}(1)-\mathrm{C}(2)$ & $112.7(3)$ & $\mathrm{C}(8)-\mathrm{C}(9)-\mathrm{C}(12)$ & $121.0(3)$ \\
\hline $\mathrm{C}(1)-\mathrm{N}(1)-\mathrm{C}(4)$ & $122.9(3)$ & $\mathrm{C}(4)-\mathrm{C}(9)-\mathrm{C}(12)$ & $121.0(3)$ \\
\hline $\mathrm{C}(2)-\mathrm{N}(1)-\mathrm{C}(4)$ & $124.3(3)$ & $\mathrm{C}(18)-\mathrm{C}(13)-\mathrm{C}(14)$ & $122.3(3)$ \\
\hline $\mathrm{C}(1)-\mathrm{N}(2)-\mathrm{C}(3)$ & $112.4(3)$ & $\mathrm{C}(18)-\mathrm{C}(13)-\mathrm{N}(2)$ & $118.5(3)$ \\
\hline C(1)-N(2)-C(13) & $123.6(3)$ & $\mathrm{C}(14)-\mathrm{C}(13)-\mathrm{N}(2)$ & $119.1(3)$ \\
\hline $\mathrm{C}(3)-\mathrm{N}(2)-\mathrm{C}(13)$ & $124.0(3)$ & $\mathrm{C}(15)-\mathrm{C}(14)-\mathrm{C}(13)$ & $117.1(3)$ \\
\hline $\mathrm{C}(22)-\mathrm{N}(3)-\mathrm{C}(23)$ & $112.6(3)$ & $C(15)-C(14)-C(19)$ & 121.1(3) \\
\hline $\mathrm{C}(22)-\mathrm{N}(3)-\mathrm{C}(25)$ & $123.4(3)$ & $\mathrm{C}(13)-\mathrm{C}(14)-\mathrm{C}(19)$ & $121.8(3)$ \\
\hline $\mathrm{C}(23)-\mathrm{N}(3)-\mathrm{C}(25)$ & $123.9(3)$ & $\mathrm{C}(16)-\mathrm{C}(15)-\mathrm{C}(14)$ & $122.5(3)$ \\
\hline $\mathrm{C}(22)-\mathrm{N}(4)-\mathrm{C}(24)$ & $112.6(3)$ & $C(17)-C(16)-C(15)$ & $118.2(3)$ \\
\hline $\mathrm{C}(22)-\mathrm{N}(4)-\mathrm{C}(34)$ & 124.1(3) & $\mathrm{C}(17)-\mathrm{C}(16)-\mathrm{C}(20)$ & $121.6(3)$ \\
\hline $\mathrm{C}(24)-\mathrm{N}(4)-\mathrm{C}(34)$ & $123.3(3)$ & $\mathrm{C}(15)-\mathrm{C}(16)-\mathrm{C}(20)$ & $120.2(3)$ \\
\hline $\mathrm{N}(2)-\mathrm{C}(1)-\mathrm{N}(1)$ & $102.0(3)$ & $\mathrm{C}(16)-\mathrm{C}(17)-\mathrm{C}(18)$ & $122.0(3)$ \\
\hline $\mathrm{N}(2)-\mathrm{C}(1)-\mathrm{Pd}$ & $128.6(2)$ & $\mathrm{C}(17)-\mathrm{C}(18)-\mathrm{C}(13)$ & $117.9(3)$ \\
\hline $\mathrm{N}(1)-\mathrm{C}(1)-\mathrm{Pd}$ & $129.5(2)$ & $\mathrm{C}(17)-\mathrm{C}(18)-\mathrm{C}(21)$ & 121.1(3) \\
\hline $\mathrm{C}(3)-\mathrm{C}(2)-\mathrm{N}(1)$ & 106.1(3) & $\mathrm{C}(13)-\mathrm{C}(18)-\mathrm{C}(21)$ & $121.0(3)$ \\
\hline $\mathrm{C}(2)-\mathrm{C}(3)-\mathrm{N}(2)$ & $106.9(3)$ & $\mathrm{N}(3)-\mathrm{C}(22)-\mathrm{N}(4)$ & $101.8(3)$ \\
\hline$C(9)-C(4)-C(5)$ & $121.9(3)$ & $\mathrm{N}(3)-\mathrm{C}(22)-\mathrm{Pd}$ & $129.8(2)$ \\
\hline $\mathrm{C}(9)-\mathrm{C}(4)-\mathrm{N}(1)$ & $118.9(3)$ & $\mathrm{N}(4)-\mathrm{C}(22)-\mathrm{Pd}$ & $128.4(2)$ \\
\hline$C(5)-C(4)-N(1)$ & $119.2(3)$ & $\mathrm{C}(24)-\mathrm{C}(23)-\mathrm{N}(3)$ & $106.4(3)$ \\
\hline $\mathrm{C}(6)-\mathrm{C}(5)-\mathrm{C}(4)$ & $117.8(3)$ & $\mathrm{C}(23)-\mathrm{C}(24)-\mathrm{N}(4)$ & $106.6(3)$ \\
\hline$C(6)-C(5)-C(10)$ & $121.2(3)$ & $\mathrm{C}(30)-\mathrm{C}(25)-\mathrm{C}(26)$ & $122.7(3)$ \\
\hline $\mathrm{C}(4)-\mathrm{C}(5)-\mathrm{C}(10)$ & $121.0(3)$ & $\mathrm{C}(30)-\mathrm{C}(25)-\mathrm{N}(3)$ & $118.8(3)$ \\
\hline$C(7)-C(6)-C(5)$ & $122.0(3)$ & $\mathrm{C}(26)-\mathrm{C}(25)-\mathrm{N}(3)$ & $118.5(3)$ \\
\hline $\mathrm{C}(6)-\mathrm{C}(7)-\mathrm{C}(8)$ & $118.3(3)$ & $\mathrm{C}(25)-\mathrm{C}(26)-\mathrm{C}(27)$ & $117.5(3)$ \\
\hline$C(6)-C(7)-C(11)$ & $121.0(3)$ & $\mathrm{C}(25)-\mathrm{C}(26)-\mathrm{C}(31)$ & $122.1(3)$ \\
\hline $\mathrm{C}(8)-\mathrm{C}(7)-\mathrm{C}(11)$ & $120.7(3)$ & $\mathrm{C}(27)-\mathrm{C}(26)-\mathrm{C}(31)$ & $120.4(3)$ \\
\hline $\mathrm{C}(9)-\mathrm{C}(8)-\mathrm{C}(7)$ & $121.9(3)$ & $\mathrm{C}(28)-\mathrm{C}(27)-\mathrm{C}(26)$ & $121.6(3)$ \\
\hline
\end{tabular}




$\begin{array}{llll}\mathrm{C}(29)-\mathrm{C}(28)-\mathrm{C}(27) & 118.7(3) & \mathrm{C}(34)-\mathrm{C}(35)-\mathrm{C}(40) & 121.3(3) \\ \mathrm{C}(29)-\mathrm{C}(28)-\mathrm{C}(32) & 121.2(4) & \mathrm{C}(36)-\mathrm{C}(35)-\mathrm{C}(40) & 120.9(3) \\ \mathrm{C}(27)-\mathrm{C}(28)-\mathrm{C}(32) & 120.1(4) & \mathrm{C}(37)-\mathrm{C}(36)-\mathrm{C}(35) & 121.7(3) \\ \mathrm{C}(28)-\mathrm{C}(29)-\mathrm{C}(30) & 121.8(3) & \mathrm{C}(36)-\mathrm{C}(37)-\mathrm{C}(38) & 118.7(3) \\ \mathrm{C}(25)-\mathrm{C}(30)-\mathrm{C}(29) & 117.7(3) & \mathrm{C}(36)-\mathrm{C}(37)-\mathrm{C}(41) & 121.1(3) \\ \mathrm{C}(25)-\mathrm{C}(30)-\mathrm{C}(33) & 121.8(3) & \mathrm{C}(38)-\mathrm{C}(37)-\mathrm{C}(41) & 120.2(3) \\ \mathrm{C}(29)-\mathrm{C}(30)-\mathrm{C}(33) & 120.4(3) & \mathrm{C}(37)-\mathrm{C}(38)-\mathrm{C}(39) & 121.9(3) \\ \mathrm{C}(35)-\mathrm{C}(34)-\mathrm{C}(39) & 122.5(3) & \mathrm{C}(34)-\mathrm{C}(39)-\mathrm{C}(38) & 117.4(3) \\ \mathrm{C}(35)-\mathrm{C}(34)-\mathrm{N}(4) & 118.8(3) & \mathrm{C}(34)-\mathrm{C}(39)-\mathrm{C}(42) & 121.6(3) \\ \mathrm{C}(39)-\mathrm{C}(34)-\mathrm{N}(4) & 118.7(3) & \mathrm{C}(38)-\mathrm{C}(39)-\mathrm{C}(42) & 120.9(3) \\ \mathrm{C}(34)-\mathrm{C}(35)-\mathrm{C}(36) & 117.8(3) & & \end{array}$

Symmetry transformations used to generate equivalent atoms: 
Table S5. Anisotropic displacement parameters $\left(\AA^{2} \times 10^{3}\right)$ for (2). The anisotropic displacement factor exponent takes the form: $-2 \pi^{2}\left[h^{2} a^{* 2} U^{11}+\ldots+2 h k a^{*} b^{*} U^{12}\right]$

\begin{tabular}{|c|c|c|c|c|c|c|}
\hline & $\mathrm{U}^{11}$ & $\mathrm{U}^{22}$ & $\mathrm{U}^{33}$ & $\mathrm{U}^{23}$ & $\mathrm{U}^{13}$ & $\mathrm{U}^{12}$ \\
\hline $\mathrm{Pd}$ & $23(1)$ & $13(1)$ & $13(1)$ & $0(1)$ & $2(1)$ & $2(1)$ \\
\hline $\mathrm{N}(1)$ & $30(2)$ & $21(2)$ & $19(2)$ & $-4(1)$ & $-2(1)$ & $5(1)$ \\
\hline $\mathrm{N}(2)$ & $29(2)$ & $17(1)$ & $20(2)$ & $-2(1)$ & $-1(1)$ & $4(1)$ \\
\hline$N(3)$ & $23(2)$ & $17(1)$ & $16(1)$ & 1(1) & $5(1)$ & 2(1) \\
\hline $\mathrm{N}(4)$ & $21(1)$ & $16(1)$ & $13(1)$ & $0(1)$ & $5(1)$ & $0(1)$ \\
\hline $\mathrm{C}(1)$ & $22(2)$ & $19(2)$ & $17(2)$ & $-1(1)$ & $0(1)$ & $-1(1)$ \\
\hline$C(2)$ & $49(3)$ & $18(2)$ & $32(2)$ & $-8(2)$ & $-4(2)$ & $10(2)$ \\
\hline$C(3)$ & $51(3)$ & $20(2)$ & $30(2)$ & $-1(2)$ & $-7(2)$ & $14(2)$ \\
\hline $\mathrm{C}(4)$ & $31(2)$ & $15(2)$ & $17(2)$ & $-4(1)$ & $1(1)$ & $3(1)$ \\
\hline C(5) & $25(2)$ & $23(2)$ & $28(2)$ & $-3(1)$ & $3(2)$ & $-4(1)$ \\
\hline C(6) & $34(2)$ & $21(2)$ & $20(2)$ & $0(1)$ & $7(2)$ & $-3(1)$ \\
\hline$C(7)$ & $31(2)$ & $18(2)$ & $16(2)$ & $-4(1)$ & $4(1)$ & 1(1) \\
\hline C(8) & $26(2)$ & $21(2)$ & $21(2)$ & $-2(1)$ & $7(1)$ & $-1(1)$ \\
\hline C(9) & $31(2)$ & $18(2)$ & $18(2)$ & $-2(1)$ & $7(1)$ & $3(1)$ \\
\hline$C(10)$ & $32(2)$ & $51(3)$ & $36(2)$ & $2(2)$ & $1(2)$ & $-13(2)$ \\
\hline $\mathrm{C}(11)$ & $33(2)$ & $31(2)$ & $18(2)$ & $-1(1)$ & $4(2)$ & $3(2)$ \\
\hline$C(12)$ & $40(2)$ & $33(2)$ & $27(2)$ & $7(2)$ & $9(2)$ & $1(2)$ \\
\hline$C(13)$ & $24(2)$ & $16(2)$ & $17(2)$ & $2(1)$ & $-4(1)$ & $4(1)$ \\
\hline$C(14)$ & $19(2)$ & $15(2)$ & $21(2)$ & $5(1)$ & $0(1)$ & $2(1)$ \\
\hline$C(15)$ & $23(2)$ & $18(2)$ & $17(2)$ & $4(1)$ & $6(1)$ & $3(1)$ \\
\hline$C(16)$ & $22(2)$ & $21(2)$ & $19(2)$ & $2(1)$ & $0(1)$ & $3(1)$ \\
\hline$C(17)$ & $17(2)$ & $28(2)$ & $20(2)$ & $-1(1)$ & 1(1) & $2(1)$ \\
\hline $\mathrm{C}(18)$ & $21(2)$ & $24(2)$ & $18(2)$ & $2(1)$ & $4(1)$ & $6(1)$ \\
\hline$C(19)$ & $26(2)$ & $27(2)$ & $32(2)$ & $2(2)$ & $5(2)$ & $-4(2)$ \\
\hline$C(20)$ & $28(2)$ & $38(2)$ & $24(2)$ & $-7(2)$ & $2(2)$ & $3(2)$ \\
\hline$C(21)$ & $30(2)$ & $40(2)$ & $22(2)$ & $-1(2)$ & $8(2)$ & $4(2)$ \\
\hline$C(22)$ & $21(2)$ & $17(2)$ & $14(2)$ & $2(1)$ & $3(1)$ & $-1(1)$ \\
\hline$C(23)$ & $27(2)$ & $15(2)$ & $23(2)$ & $4(1)$ & $5(1)$ & $5(1)$ \\
\hline$C(24)$ & $23(2)$ & $17(2)$ & $24(2)$ & 1(1) & $7(1)$ & $4(1)$ \\
\hline$C(25)$ & $25(2)$ & $19(2)$ & $14(2)$ & $5(1)$ & $5(1)$ & $6(1)$ \\
\hline$C(26)$ & $26(2)$ & $21(2)$ & $16(2)$ & $5(1)$ & 1(1) & $5(1)$ \\
\hline$C(27)$ & $32(2)$ & $24(2)$ & $17(2)$ & $1(1)$ & 1(1) & $3(2)$ \\
\hline $\mathrm{C}(28)$ & $33(2)$ & $25(2)$ & $18(2)$ & $6(1)$ & $8(2)$ & $10(2)$ \\
\hline$C(29)$ & $24(2)$ & $26(2)$ & $25(2)$ & $7(1)$ & $7(1)$ & $3(1)$ \\
\hline$C(30)$ & $27(2)$ & $20(2)$ & $19(2)$ & $4(1)$ & $6(1)$ & $4(1)$ \\
\hline $\mathrm{C}(31)$ & $26(2)$ & $32(2)$ & $20(2)$ & $0(2)$ & $2(1)$ & $-4(2)$ \\
\hline $\mathrm{C}(32)$ & $40(2)$ & $47(2)$ & $23(2)$ & $2(2)$ & $13(2)$ & $9(2)$ \\
\hline $\mathrm{C}(33)$ & $27(2)$ & $25(2)$ & $25(2)$ & $-1(1)$ & $5(2)$ & $-1(1)$ \\
\hline$C(34)$ & $20(2)$ & $15(2)$ & $16(2)$ & $0(1)$ & $7(1)$ & $1(1)$ \\
\hline$C(35)$ & $21(2)$ & $19(2)$ & $19(2)$ & $-4(1)$ & $5(1)$ & 1(1) \\
\hline$C(36)$ & $20(2)$ & $22(2)$ & $24(2)$ & $-1(1)$ & $11(1)$ & $-3(1)$ \\
\hline $\mathrm{C}(37)$ & $27(2)$ & $18(2)$ & $17(2)$ & $0(1)$ & $7(1)$ & $0(1)$ \\
\hline $\mathrm{C}(38)$ & $24(2)$ & $16(2)$ & $14(2)$ & $-2(1)$ & 1(1) & $2(1)$ \\
\hline$C(39)$ & $19(2)$ & $12(1)$ & $22(2)$ & $-4(1)$ & $6(1)$ & 1(1) \\
\hline $\mathrm{C}(40)$ & $23(2)$ & $32(2)$ & $24(2)$ & $0(2)$ & 1(1) & $0(2)$ \\
\hline $\mathrm{C}(41)$ & $33(2)$ & $33(2)$ & $22(2)$ & $5(2)$ & $9(2)$ & $-4(2)$ \\
\hline $\mathrm{C}(42)$ & $18(2)$ & $24(2)$ & $24(2)$ & $-1(1)$ & $6(1)$ & $-1(1)$ \\
\hline
\end{tabular}


Table S6. Hydrogen coordinates ( x $\left.10^{4}\right)$ and isotropic displacement parameters $\left(\AA^{2} \times 10^{3}\right)$ for (2).

\begin{tabular}{|c|c|c|c|c|}
\hline & $\mathrm{x}$ & $\mathrm{y}$ & $\mathrm{z}$ & $\mathrm{U}(\mathrm{eq})$ \\
\hline $\mathrm{H}(2)$ & 1930 & 3046 & 6545 & 41 \\
\hline $\mathrm{H}(3)$ & 1191 & 2806 & 4944 & 42 \\
\hline $\mathrm{H}(6)$ & 2883 & 1013 & 9447 & 30 \\
\hline $\mathrm{H}(8)$ & 4854 & 2002 & 8352 & 27 \\
\hline $\mathrm{H}(10 \mathrm{~A})$ & 1534 & 629 & 7556 & 60 \\
\hline $\mathrm{H}(10 \mathrm{~B})$ & 1234 & 1530 & 7769 & 60 \\
\hline $\mathrm{H}(10 \mathrm{C})$ & 1482 & 898 & 8600 & 60 \\
\hline $\mathrm{H}(11 \mathrm{~A})$ & 5021 & 904 & 9779 & 42 \\
\hline $\mathrm{H}(11 \mathrm{~B})$ & 4247 & 1036 & 10366 & 42 \\
\hline $\mathrm{H}(11 \mathrm{C})$ & 4842 & 1788 & 10168 & 42 \\
\hline $\mathrm{H}(12 \mathrm{~A})$ & 3890 & 2085 & 6088 & 49 \\
\hline $\mathrm{H}(12 \mathrm{~B})$ & 4703 & 2414 & 6788 & 49 \\
\hline $\mathrm{H}(12 \mathrm{C})$ & 3838 & 2944 & 6591 & 49 \\
\hline $\mathrm{H}(15)$ & 1853 & 721 & 2272 & 23 \\
\hline $\mathrm{H}(17)$ & -441 & 465 & 3168 & 26 \\
\hline $\mathrm{H}(19 \mathrm{~A})$ & 2877 & 1924 & 3980 & 43 \\
\hline $\mathrm{H}(19 \mathrm{~B})$ & 3064 & 1303 & 3189 & 43 \\
\hline $\mathrm{H}(19 \mathrm{C})$ & 3119 & 998 & 4238 & 43 \\
\hline $\mathrm{H}(20 \mathrm{~A})$ & 442 & -437 & 1686 & 45 \\
\hline $\mathrm{H}(20 \mathrm{~B})$ & 540 & 405 & 1164 & 45 \\
\hline $\mathrm{H}(20 \mathrm{C})$ & -339 & 200 & 1553 & 45 \\
\hline $\mathrm{H}(21 \mathrm{~A})$ & -640 & 809 & 4684 & 46 \\
\hline $\mathrm{H}(21 \mathrm{~B})$ & -158 & 1639 & 5016 & 46 \\
\hline $\mathrm{H}(21 \mathrm{C})$ & 213 & 791 & 5443 & 46 \\
\hline $\mathrm{H}(23)$ & 3283 & -2881 & 6778 & 26 \\
\hline $\mathrm{H}(24)$ & 3659 & -2741 & 5196 & 25 \\
\hline $\mathrm{H}(27)$ & 3097 & -557 & 9465 & 30 \\
\hline $\mathrm{H}(29)$ & 957 & -1857 & 8548 & 29 \\
\hline $\mathrm{H}(31 \mathrm{~A})$ & 4301 & -1058 & 7788 & 39 \\
\hline $\mathrm{H}(31 \mathrm{~B})$ & 4227 & -408 & 8588 & 39 \\
\hline $\mathrm{H}(31 \mathrm{C})$ & 3822 & -209 & 7538 & 39 \\
\hline $\mathrm{H}(32 \mathrm{~A})$ & 1647 & -422 & 10154 & 54 \\
\hline $\mathrm{H}(32 \mathrm{~B})$ & 1999 & -1285 & 10551 & 54 \\
\hline $\mathrm{H}(32 \mathrm{C})$ & 1039 & -1207 & 9996 & 54 \\
\hline $\mathrm{H}(33 \mathrm{~A})$ & 1307 & -2093 & 6276 & 38 \\
\hline $\mathrm{H}(33 \mathrm{~B})$ & 681 & -2345 & 7001 & 38 \\
\hline $\mathrm{H}(33 \mathrm{C})$ & 1519 & -2880 & 6914 & 38 \\
\hline $\mathrm{H}(36)$ & 4964 & -607 & 3314 & 26 \\
\hline $\mathrm{H}(38)$ & 2413 & -739 & 2354 & 22 \\
\hline $\mathrm{H}(40 \mathrm{~A})$ & 5518 & -870 & 4876 & 40 \\
\hline $\mathrm{H}(40 \mathrm{~B})$ & 5093 & -1680 & 5214 & 40 \\
\hline $\mathrm{H}(40 \mathrm{C})$ & 4854 & -815 & 5608 & 40 \\
\hline $\mathrm{H}(41 \mathrm{~A})$ & 3607 & -663 & 1272 & 43 \\
\hline $\mathrm{H}(41 \mathrm{~B})$ & 4492 & -258 & 1753 & 43 \\
\hline $\mathrm{H}(41 \mathrm{C})$ & 3608 & 245 & 1667 & 43 \\
\hline $\mathrm{H}(42 \mathrm{~A})$ & 1747 & -1743 & 4175 & 33 \\
\hline $\mathrm{H}(42 \mathrm{~B})$ & 1402 & -1201 & 3285 & 33 \\
\hline $\mathrm{H}(42 \mathrm{C})$ & 1635 & -783 & 4282 & 33 \\
\hline
\end{tabular}


Table S7. Torsion angles $\left[{ }^{\circ}\right]$ for (2).

\begin{tabular}{|c|c|c|c|}
\hline $\bar{C}(3)-N(2)-C(1)-N(1)$ & $0.1(4)$ & $\mathrm{C}(25)-\mathrm{N}(3)-\mathrm{C}(22)-\mathrm{N}(4)$ & $-177.0(3)$ \\
\hline $\mathrm{C}(13)-\mathrm{N}(2)-\mathrm{C}(1)-\mathrm{N}(1)$ & $-178.8(3)$ & $\mathrm{C}(23)-\mathrm{N}(3)-\mathrm{C}(22)-\mathrm{Pd}$ & $179.4(2)$ \\
\hline $\mathrm{C}(3)-\mathrm{N}(2)-\mathrm{C}(1)-\mathrm{Pd}$ & $178.6(3)$ & $\mathrm{C}(25)-\mathrm{N}(3)-\mathrm{C}(22)-\mathrm{Pd}$ & $2.0(5)$ \\
\hline $\mathrm{C}(13)-\mathrm{N}(2)-\mathrm{C}(1)-\mathrm{Pd}$ & $-0.3(5)$ & $\mathrm{C}(24)-\mathrm{N}(4)-\mathrm{C}(22)-\mathrm{N}(3)$ & $-0.2(4)$ \\
\hline $\mathrm{C}(2)-\mathrm{N}(1)-\mathrm{C}(1)-\mathrm{N}(2)$ & $0.3(4)$ & $\mathrm{C}(34)-\mathrm{N}(4)-\mathrm{C}(22)-\mathrm{N}(3)$ & $-178.9(3)$ \\
\hline $\mathrm{C}(4)-\mathrm{N}(1)-\mathrm{C}(1)-\mathrm{N}(2)$ & $-175.9(3)$ & $\mathrm{C}(24)-\mathrm{N}(4)-\mathrm{C}(22)-\mathrm{Pd}$ & $-179.2(2)$ \\
\hline $\mathrm{C}(2)-\mathrm{N}(1)-\mathrm{C}(1)-\mathrm{Pd}$ & $-178.2(3)$ & $\mathrm{C}(34)-\mathrm{N}(4)-\mathrm{C}(22)-\mathrm{Pd}$ & $2.1(5)$ \\
\hline $\mathrm{C}(4)-\mathrm{N}(1)-\mathrm{C}(1)-\mathrm{Pd}$ & $5.6(5)$ & $\mathrm{C}(1)-\mathrm{Pd}-\mathrm{C}(22)-\mathrm{N}(3)$ & $-103(7)$ \\
\hline $\mathrm{C}(22)-\mathrm{Pd}-\mathrm{C}(1)-\mathrm{N}(2)$ & $-31(7)$ & $\mathrm{C}(1)-\mathrm{Pd}-\mathrm{C}(22)-\mathrm{N}(4)$ & $75(7)$ \\
\hline $\mathrm{C}(22)-\mathrm{Pd}-\mathrm{C}(1)-\mathrm{N}(1)$ & $148(6)$ & $\mathrm{C}(22)-\mathrm{N}(3)-\mathrm{C}(23)-\mathrm{C}(24)$ & $-0.5(4)$ \\
\hline $\mathrm{C}(1)-\mathrm{N}(1)-\mathrm{C}(2)-\mathrm{C}(3)$ & $-0.5(5)$ & $\mathrm{C}(25)-\mathrm{N}(3)-\mathrm{C}(23)-\mathrm{C}(24)$ & $176.9(3)$ \\
\hline $\mathrm{C}(4)-\mathrm{N}(1)-\mathrm{C}(2)-\mathrm{C}(3)$ & $175.6(4)$ & $\mathrm{N}(3)-\mathrm{C}(23)-\mathrm{C}(24)-\mathrm{N}(4)$ & $0.3(4)$ \\
\hline $\mathrm{N}(1)-\mathrm{C}(2)-\mathrm{C}(3)-\mathrm{N}(2)$ & $0.6(5)$ & $\mathrm{C}(22)-\mathrm{N}(4)-\mathrm{C}(24)-\mathrm{C}(23)$ & $-0.1(4)$ \\
\hline $\mathrm{C}(1)-\mathrm{N}(2)-\mathrm{C}(3)-\mathrm{C}(2)$ & $-0.5(5)$ & $\mathrm{C}(34)-\mathrm{N}(4)-\mathrm{C}(24)-\mathrm{C}(23)$ & $178.6(3)$ \\
\hline$C(13)-N(2)-C(3)-C(2)$ & $178.5(4)$ & $\mathrm{C}(22)-\mathrm{N}(3)-\mathrm{C}(25)-\mathrm{C}(30)$ & $106.4(4)$ \\
\hline $\mathrm{C}(1)-\mathrm{N}(1)-\mathrm{C}(4)-\mathrm{C}(9)$ & $95.5(4)$ & $\mathrm{C}(23)-\mathrm{N}(3)-\mathrm{C}(25)-\mathrm{C}(30)$ & $-70.7(4)$ \\
\hline $\mathrm{C}(2)-\mathrm{N}(1)-\mathrm{C}(4)-\mathrm{C}(9)$ & $-80.2(5)$ & $\mathrm{C}(22)-\mathrm{N}(3)-\mathrm{C}(25)-\mathrm{C}(26)$ & $-74.5(4)$ \\
\hline $\mathrm{C}(1)-\mathrm{N}(1)-\mathrm{C}(4)-\mathrm{C}(5)$ & $-84.4(4)$ & $\mathrm{C}(23)-\mathrm{N}(3)-\mathrm{C}(25)-\mathrm{C}(26)$ & $108.4(4)$ \\
\hline $\mathrm{C}(2)-\mathrm{N}(1)-\mathrm{C}(4)-\mathrm{C}(5)$ & $99.8(4)$ & $\mathrm{C}(30)-\mathrm{C}(25)-\mathrm{C}(26)-\mathrm{C}(27)$ & $0.1(5)$ \\
\hline $\mathrm{C}(9)-\mathrm{C}(4)-\mathrm{C}(5)-\mathrm{C}(6)$ & $-1.5(5)$ & $\mathrm{N}(3)-\mathrm{C}(25)-\mathrm{C}(26)-\mathrm{C}(27)$ & $-179.0(3)$ \\
\hline $\mathrm{N}(1)-\mathrm{C}(4)-\mathrm{C}(5)-\mathrm{C}(6)$ & $178.4(3)$ & $\mathrm{C}(30)-\mathrm{C}(25)-\mathrm{C}(26)-\mathrm{C}(31)$ & $-178.1(3)$ \\
\hline$C(9)-C(4)-C(5)-C(10)$ & $-179.3(4)$ & $\mathrm{N}(3)-\mathrm{C}(25)-\mathrm{C}(26)-\mathrm{C}(31)$ & $2.8(5)$ \\
\hline $\mathrm{N}(1)-\mathrm{C}(4)-\mathrm{C}(5)-\mathrm{C}(10)$ & $0.6(5)$ & $\mathrm{C}(25)-\mathrm{C}(26)-\mathrm{C}(27)-\mathrm{C}(28)$ & $-0.9(5)$ \\
\hline$C(4)-C(5)-C(6)-C(7)$ & $-1.1(5)$ & $\mathrm{C}(31)-\mathrm{C}(26)-\mathrm{C}(27)-\mathrm{C}(28)$ & $177.3(3)$ \\
\hline $\mathrm{C}(10)-\mathrm{C}(5)-\mathrm{C}(6)-\mathrm{C}(7)$ & $176.7(4)$ & $\mathrm{C}(26)-\mathrm{C}(27)-\mathrm{C}(28)-\mathrm{C}(29)$ & $0.7(5)$ \\
\hline$C(5)-C(6)-C(7)-C(8)$ & $2.6(5)$ & $\mathrm{C}(26)-\mathrm{C}(27)-\mathrm{C}(28)-\mathrm{C}(32)$ & $-179.1(3)$ \\
\hline$C(5)-C(6)-C(7)-C(11)$ & $-175.8(3)$ & $\mathrm{C}(27)-\mathrm{C}(28)-\mathrm{C}(29)-\mathrm{C}(30)$ & $0.4(5)$ \\
\hline $\mathrm{C}(6)-\mathrm{C}(7)-\mathrm{C}(8)-\mathrm{C}(9)$ & $-1.6(5)$ & $\mathrm{C}(32)-\mathrm{C}(28)-\mathrm{C}(29)-\mathrm{C}(30)$ & $-179.8(3)$ \\
\hline $\mathrm{C}(11)-\mathrm{C}(7)-\mathrm{C}(8)-\mathrm{C}(9)$ & $176.8(3)$ & $\mathrm{C}(26)-\mathrm{C}(25)-\mathrm{C}(30)-\mathrm{C}(29)$ & $0.9(5)$ \\
\hline$C(7)-C(8)-C(9)-C(4)$ & $-0.9(5)$ & $\mathrm{N}(3)-\mathrm{C}(25)-\mathrm{C}(30)-\mathrm{C}(29)$ & $180.0(3)$ \\
\hline $\mathrm{C}(7)-\mathrm{C}(8)-\mathrm{C}(9)-\mathrm{C}(12)$ & $179.2(3)$ & $C(26)-C(25)-C(30)-C(33)$ & $-179.7(3)$ \\
\hline$C(5)-C(4)-C(9)-C(8)$ & $2.5(5)$ & $\mathrm{N}(3)-\mathrm{C}(25)-\mathrm{C}(30)-\mathrm{C}(33)$ & $-0.6(5)$ \\
\hline $\mathrm{N}(1)-\mathrm{C}(4)-\mathrm{C}(9)-\mathrm{C}(8)$ & $-177.4(3)$ & $\mathrm{C}(28)-\mathrm{C}(29)-\mathrm{C}(30)-\mathrm{C}(25)$ & $-1.2(5)$ \\
\hline $\mathrm{C}(5)-\mathrm{C}(4)-\mathrm{C}(9)-\mathrm{C}(12)$ & $-177.6(3)$ & $\mathrm{C}(28)-\mathrm{C}(29)-\mathrm{C}(30)-\mathrm{C}(33)$ & $179.4(3)$ \\
\hline $\mathrm{N}(1)-\mathrm{C}(4)-\mathrm{C}(9)-\mathrm{C}(12)$ & $2.4(5)$ & $\mathrm{C}(22)-\mathrm{N}(4)-\mathrm{C}(34)-\mathrm{C}(35)$ & $102.9(4)$ \\
\hline $\mathrm{C}(1)-\mathrm{N}(2)-\mathrm{C}(13)-\mathrm{C}(18)$ & $100.9(4)$ & $\mathrm{C}(24)-\mathrm{N}(4)-\mathrm{C}(34)-\mathrm{C}(35)$ & $-75.6(4)$ \\
\hline $\mathrm{C}(3)-\mathrm{N}(2)-\mathrm{C}(13)-\mathrm{C}(18)$ & $-77.9(5)$ & $\mathrm{C}(22)-\mathrm{N}(4)-\mathrm{C}(34)-\mathrm{C}(39)$ & $-77.6(4)$ \\
\hline $\mathrm{C}(1)-\mathrm{N}(2)-\mathrm{C}(13)-\mathrm{C}(14)$ & $-79.9(4)$ & $\mathrm{C}(24)-\mathrm{N}(4)-\mathrm{C}(34)-\mathrm{C}(39)$ & $103.8(4)$ \\
\hline $\mathrm{C}(3)-\mathrm{N}(2)-\mathrm{C}(13)-\mathrm{C}(14)$ & $101.2(4)$ & $C(39)-C(34)-C(35)-C(36)$ & $-1.3(5)$ \\
\hline $\mathrm{C}(18)-\mathrm{C}(13)-\mathrm{C}(14)-\mathrm{C}(15)$ & $1.3(5)$ & $\mathrm{N}(4)-\mathrm{C}(34)-\mathrm{C}(35)-\mathrm{C}(36)$ & $178.1(3)$ \\
\hline $\mathrm{N}(2)-\mathrm{C}(13)-\mathrm{C}(14)-\mathrm{C}(15)$ & $-177.8(3)$ & $\mathrm{C}(39)-\mathrm{C}(34)-\mathrm{C}(35)-\mathrm{C}(40)$ & $176.0(3)$ \\
\hline $\mathrm{C}(18)-\mathrm{C}(13)-\mathrm{C}(14)-\mathrm{C}(19)$ & $-175.9(3)$ & $\mathrm{N}(4)-\mathrm{C}(34)-\mathrm{C}(35)-\mathrm{C}(40)$ & $-4.6(5)$ \\
\hline $\mathrm{N}(2)-\mathrm{C}(13)-\mathrm{C}(14)-\mathrm{C}(19)$ & $4.9(5)$ & $C(34)-C(35)-C(36)-C(37)$ & $0.1(5)$ \\
\hline$C(13)-C(14)-C(15)-C(16)$ & $-0.9(5)$ & $\mathrm{C}(40)-\mathrm{C}(35)-\mathrm{C}(36)-\mathrm{C}(37)$ & $-177.2(3)$ \\
\hline $\mathrm{C}(19)-\mathrm{C}(14)-\mathrm{C}(15)-\mathrm{C}(16)$ & $176.3(3)$ & $\mathrm{C}(35)-\mathrm{C}(36)-\mathrm{C}(37)-\mathrm{C}(38)$ & $0.1(5)$ \\
\hline $\mathrm{C}(14)-\mathrm{C}(15)-\mathrm{C}(16)-\mathrm{C}(17)$ & $0.2(5)$ & $\mathrm{C}(35)-\mathrm{C}(36)-\mathrm{C}(37)-\mathrm{C}(41)$ & $179.2(3)$ \\
\hline$C(14)-C(15)-C(16)-C(20)$ & $-178.5(3)$ & $\mathrm{C}(36)-\mathrm{C}(37)-\mathrm{C}(38)-\mathrm{C}(39)$ & $0.9(5)$ \\
\hline $\mathrm{C}(15)-\mathrm{C}(16)-\mathrm{C}(17)-\mathrm{C}(18)$ & $0.1(5)$ & $\mathrm{C}(41)-\mathrm{C}(37)-\mathrm{C}(38)-\mathrm{C}(39)$ & $-178.2(3)$ \\
\hline $\mathrm{C}(20)-\mathrm{C}(16)-\mathrm{C}(17)-\mathrm{C}(18)$ & $178.9(3)$ & $\mathrm{C}(35)-\mathrm{C}(34)-\mathrm{C}(39)-\mathrm{C}(38)$ & $2.3(5)$ \\
\hline $\mathrm{C}(16)-\mathrm{C}(17)-\mathrm{C}(18)-\mathrm{C}(13)$ & $0.3(5)$ & $\mathrm{N}(4)-\mathrm{C}(34)-\mathrm{C}(39)-\mathrm{C}(38)$ & $-177.2(3)$ \\
\hline $\mathrm{C}(16)-\mathrm{C}(17)-\mathrm{C}(18)-\mathrm{C}(21)$ & $-177.6(3)$ & $\mathrm{C}(35)-\mathrm{C}(34)-\mathrm{C}(39)-\mathrm{C}(42)$ & $-174.5(3)$ \\
\hline $\mathrm{C}(14)-\mathrm{C}(13)-\mathrm{C}(18)-\mathrm{C}(17)$ & $-1.0(5)$ & $\mathrm{N}(4)-\mathrm{C}(34)-\mathrm{C}(39)-\mathrm{C}(42)$ & $6.1(4)$ \\
\hline $\mathrm{N}(2)-\mathrm{C}(13)-\mathrm{C}(18)-\mathrm{C}(17)$ & $178.1(3)$ & $\mathrm{C}(37)-\mathrm{C}(38)-\mathrm{C}(39)-\mathrm{C}(34)$ & $-2.0(5)$ \\
\hline $\mathrm{C}(14)-\mathrm{C}(13)-\mathrm{C}(18)-\mathrm{C}(21)$ & $176.8(3)$ & $\mathrm{C}(37)-\mathrm{C}(38)-\mathrm{C}(39)-\mathrm{C}(42)$ & $174.7(3)$ \\
\hline $\mathrm{N}(2)-\mathrm{C}(13)-\mathrm{C}(18)-\mathrm{C}(21)$ & $-4.0(5)$ & & \\
\hline $\mathrm{C}(23)-\mathrm{N}(3)-\mathrm{C}(22)-\mathrm{N}(4)$ & $0.4(4)$ & & \\
\hline
\end{tabular}


Symmetry transformations used to generate equivalent atoms: 


\section{X-ray crystallographic Analysis of $\mathrm{Pd}(\mathrm{IMes})_{2}\left(\mathrm{O}_{2}\right),(3)$.}

A dichromic pink/light brown air-sensitive crystal with approximate dimensions $0.43 \mathrm{x}$ $0.41 \times 0.33 \mathrm{~mm}^{3}$ was selected under oil under ambient conditions and attached to the tip of a glass capillary. The crystal was mounted in a stream of cold nitrogen at 100(2) K and centered in the X-ray beam by using a video camera.

The crystal evaluation and data collection were performed on a Bruker CCD-1000 diffractometer with $\mathrm{Mo} \mathrm{K}_{\alpha}(\lambda=0.71073 \AA)$ radiation and the diffractometer to crystal distance of $4.9 \mathrm{~cm}$.

The initial cell constants were obtained from three series of $\omega$ scans at different starting angles. Each series consisted of 20 frames collected at intervals of $0.3^{\circ}$ in a $6^{\circ}$ range about $\omega$ with the exposure time of 10 seconds per frame. A total of 87 reflections was obtained. The reflections were successfully indexed by an automated indexing routine built in the SMART program. The final cell constants were calculated from a set of 16851 strong reflections from the actual data collection.

The data were collected by using the hemisphere data collection routine. The reciprocal space was surveyed to the extent of a full sphere to a resolution of $0.80 \AA$. A total of 36033 data were harvested by collecting three sets of frames with $0.3^{\circ}$ scans in $\omega$ with an exposure time 30 sec per frame. These highly redundant datasets were corrected for Lorentz and polarization effects. The absorption correction was based on fitting a function to the empirical transmission surface as sampled by multiple equivalent measurements ${ }^{3}$.

\section{Structure Solution and Refinement}

The systematic absences in the diffraction data were uniquely consistent for the space group $P 2{ }_{1} / \mathrm{n}$ that yielded chemically reasonable and computationally stable results of refinement ${ }^{3}$.

A successful solution by the direct methods provided most non-hydrogen atoms from the $E$-map. The remaining non-hydrogen atoms were located in an alternating series of least-squares cycles and difference Fourier maps. All non-hydrogen atoms were refined with anisotropic displacement coefficients. All hydrogen atoms were included in the structure factor calculation 
at idealized positions and were allowed to ride on the neighboring atoms with relative isotropic displacement coefficients.

There were also two halves of toluene solvate molecules present in the asymmetric unit. A significant amount of time was invested in identifying and refining the disordered molecule. Bond length restraints were applied to model the molecules but the resulting isotropic displacement coefficients suggested the molecules were mobile. In addition, the refinement was computationally unstable. Option SQUEEZE of program PLATON ${ }^{4}$ was used to correct the diffraction data for diffuse scattering effects and to identify the solvate molecules. PLATON calculated the upper limit of volume that can be occupied by the solvent to be $926.0 \AA^{3}$, or $21 \%$ of the unit cell volume. The composition corresponds to one solvate toluene molecule per Pd complex. Note that all derived results in the following tables are based on known contents. No data are given for the diffusely scattering species.

The final least-squares refinement of 461 parameters against 8990 data resulted in residuals $R$ (based on $F^{2}$ for $I \geq 2 \sigma$ ) and $w R$ (based on $F^{2}$ for all data) of 0.0297 and 0.0832 , respectively. The final difference Fourier map was featureless.

The ORTEP diagrams are drawn with $50 \%$ probability ellipsoids.

${ }^{4}$ Spek, A.L. Acta Cryst., 1990, A46,C-34. 


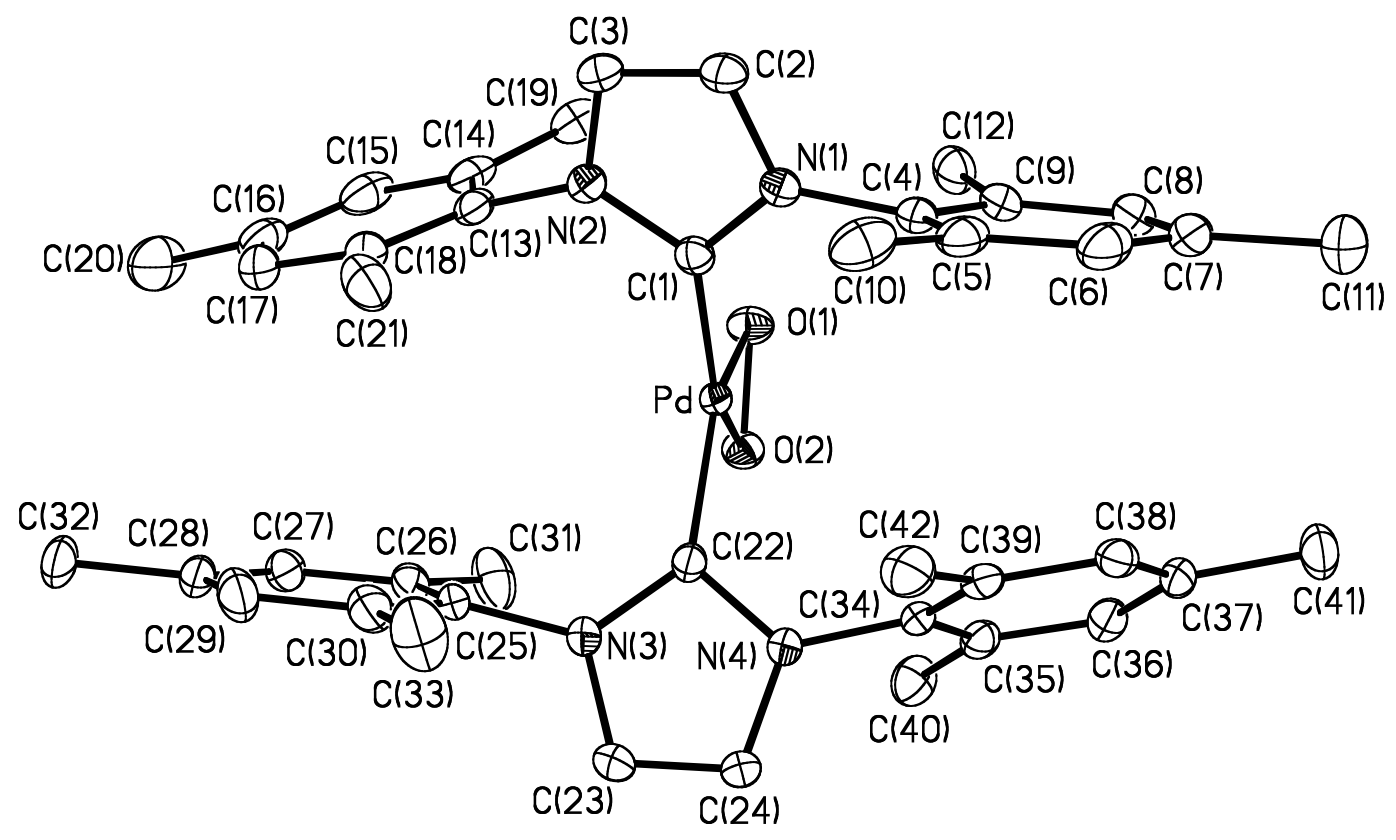

Figure S13. A molecular drawing of (3) with 50\% probability ellipsoids. 


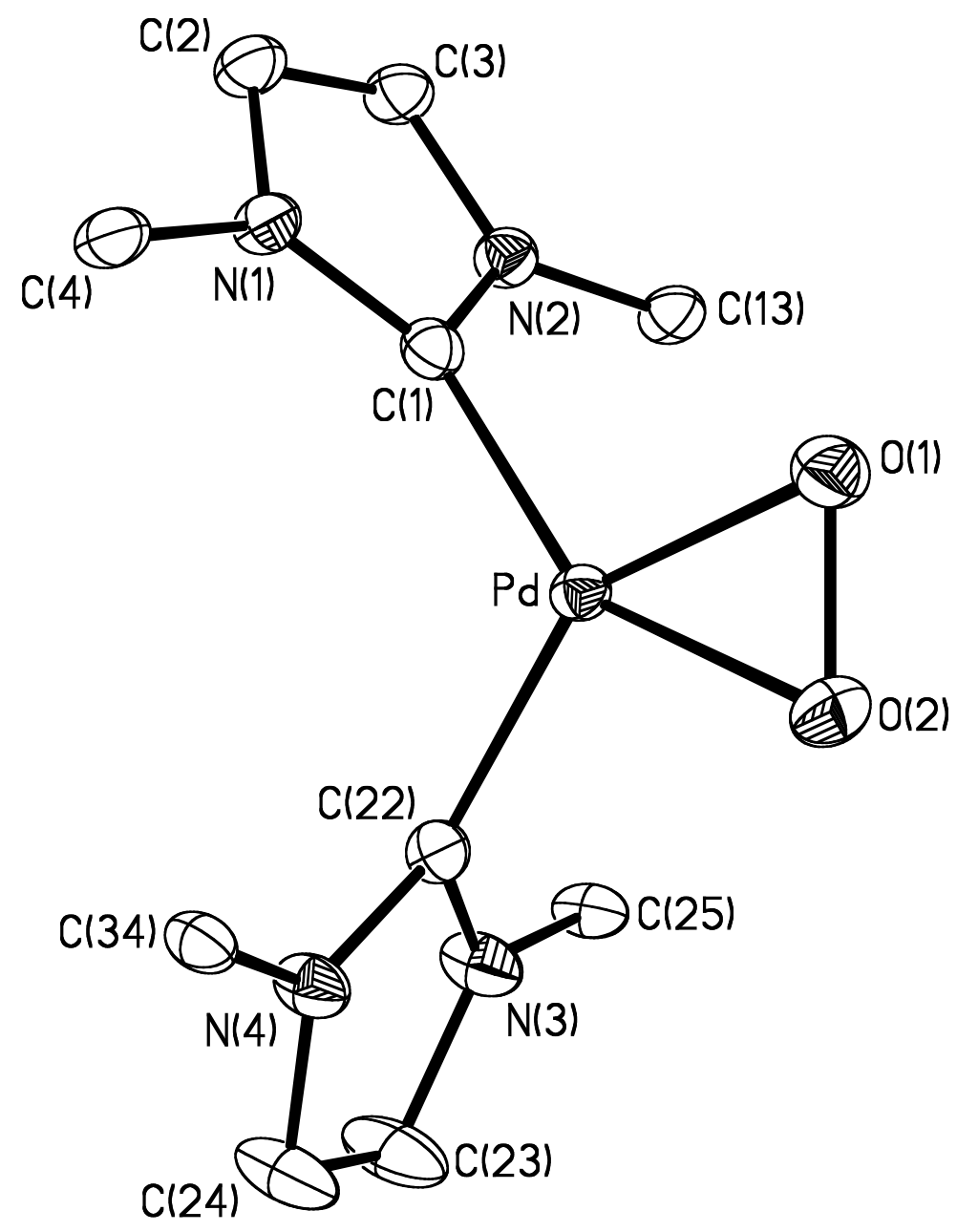

Figure S14. A molecular drawing of (3) with 50\% probability ellipsoids. The mesityl rings on the carbene ligands are removed for clarity except for the ipso carbons. 


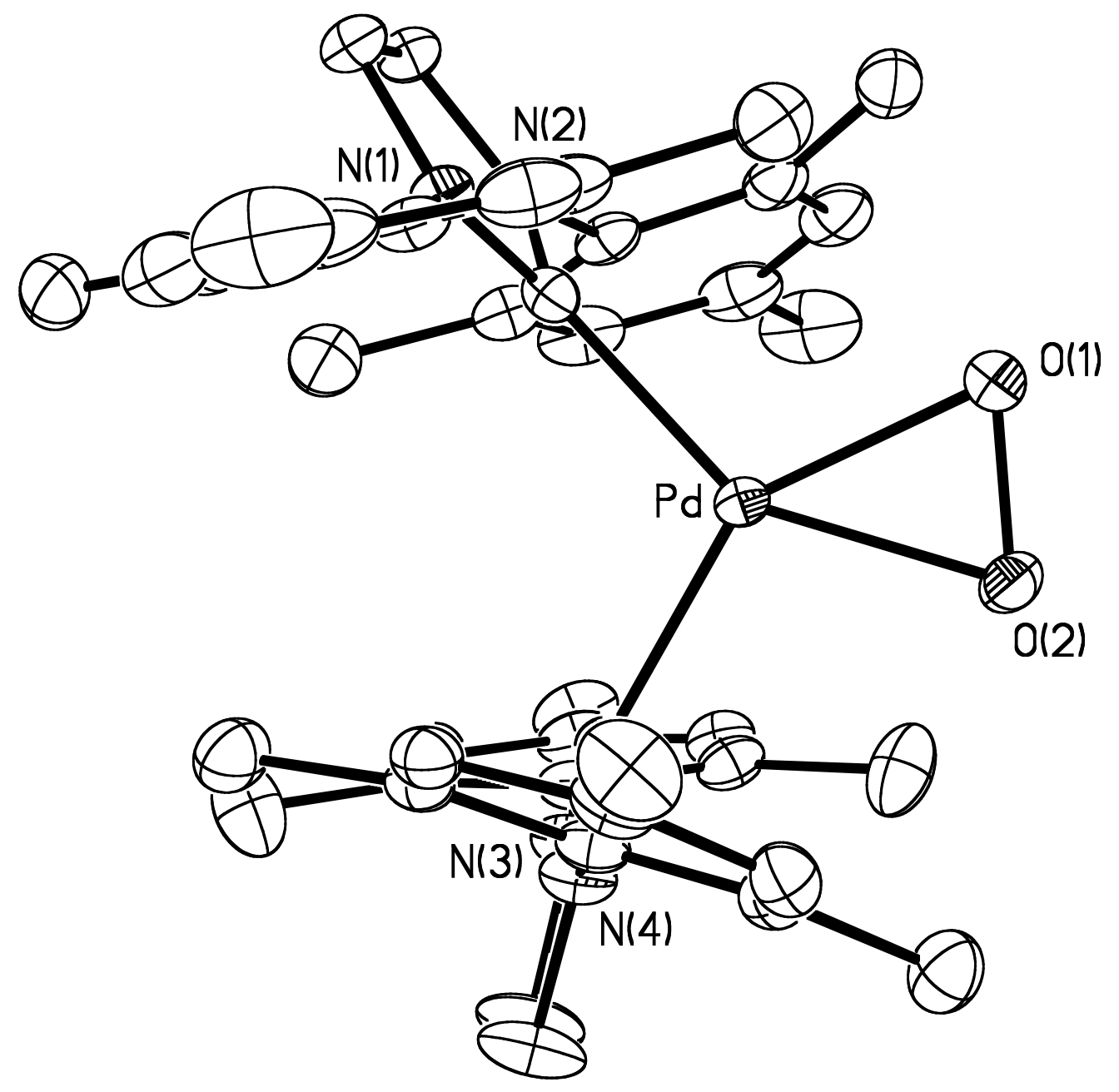

Figure S15. A view along the vector perpendicular to the $\mathrm{Pd}-\mathrm{O}(1)-\mathrm{O}(2)$ plane. The atoms are shown with $50 \%$ probability ellipsoids. 


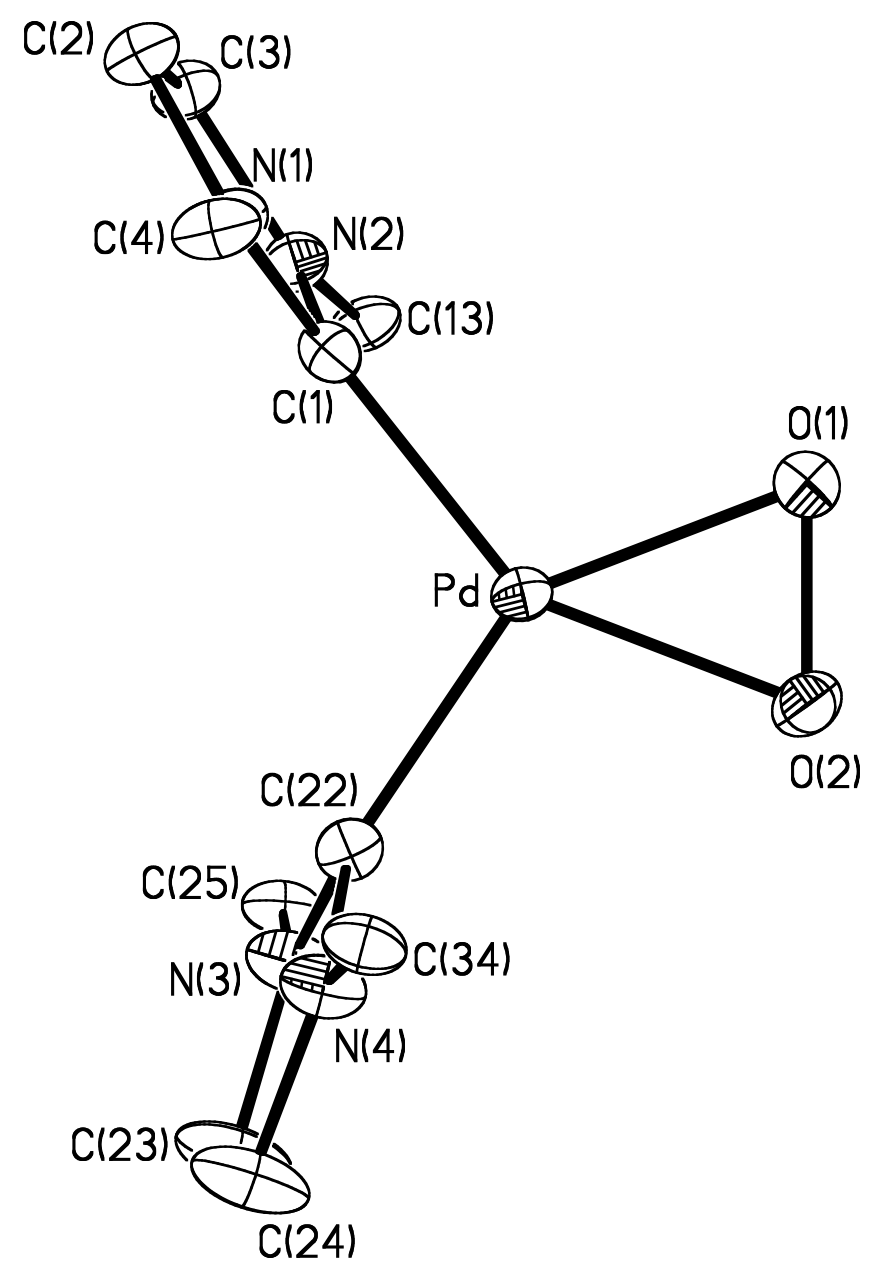

Figure S16. A view along the vector perpendicular to the $\mathrm{Pd}-\mathrm{O}(1)-\mathrm{O}(2)$ plane. The mesityl rings on the carbene ligands are removed for clarity except for the ipso carbons. $50 \%$ probability ellipsoids. 
Table S8. Crystal data and structure refinement for (3).

\begin{tabular}{|c|c|c|}
\hline Identification code & \multicolumn{2}{|l|}{ sta 10} \\
\hline Empirical formula & \multicolumn{2}{|c|}{$\mathrm{C}_{42} \mathrm{H}_{48} \mathrm{~N}_{4} \mathrm{O}_{2} \mathrm{Pd} . \mathrm{C}_{7} \mathrm{H}_{8}$} \\
\hline Formula weight & \multicolumn{2}{|l|}{839.38} \\
\hline Temperature & \multicolumn{2}{|l|}{$100(2) \mathrm{K}$} \\
\hline Wavelength & \multicolumn{2}{|l|}{$0.71073 \AA$} \\
\hline Crystal system & \multicolumn{2}{|l|}{ Monoclinic } \\
\hline Space group & \multicolumn{2}{|l|}{$\mathrm{P} 2{ }_{1} / \mathrm{n}$} \\
\hline \multirow[t]{3}{*}{ Unit cell dimensions } & $\mathrm{a}=13.3902(11) \AA$ & $\alpha=90^{\circ}$ \\
\hline & $\mathrm{b}=14.6410(12) \AA$ & $\beta=98.1911(14)^{\circ}$. \\
\hline & $\mathrm{c}=22.6735(18) \AA$ & $\gamma=90^{\circ}$. \\
\hline Volume & \multicolumn{2}{|l|}{$4399.7(6) \AA^{3}$} \\
\hline $\mathrm{Z}$ & \multicolumn{2}{|l|}{4} \\
\hline Density (calculated) & \multicolumn{2}{|l|}{$1.267 \mathrm{Mg} / \mathrm{m}^{3}$} \\
\hline Absorption coefficient & \multicolumn{2}{|l|}{$0.464 \mathrm{~mm}^{-1}$} \\
\hline $\mathrm{F}(000)$ & \multicolumn{2}{|l|}{1760} \\
\hline Crystal size & \multicolumn{2}{|c|}{$0.43 \times 0.41 \times 0.33 \mathrm{~mm}^{3}$} \\
\hline Theta range for data collection & \multicolumn{2}{|l|}{1.66 to $26.38^{\circ}$. } \\
\hline Index ranges & \multicolumn{2}{|c|}{$-16<=\mathrm{h}<=16,-18<=\mathrm{k}<=18,-28<=1<=28$} \\
\hline Reflections collected & \multicolumn{2}{|l|}{36033} \\
\hline Independent reflections & \multicolumn{2}{|c|}{$8990[\mathrm{R}(\mathrm{int})=0.0327]$} \\
\hline Completeness to theta $=26.38^{\circ}$ & \multicolumn{2}{|l|}{$99.7 \%$} \\
\hline Absorption correction & \multicolumn{2}{|c|}{ Empirical with SADABS } \\
\hline Max. and min. transmission & \multicolumn{2}{|l|}{0.8620 and 0.8255} \\
\hline Refinement method & \multicolumn{2}{|c|}{ Full-matrix least-squares on $\mathrm{F}^{2}$} \\
\hline Data / restraints / parameters & \multicolumn{2}{|l|}{8990 / 0 / 461} \\
\hline Goodness-of-fit on $\mathrm{F}^{2}$ & \multicolumn{2}{|l|}{1.093} \\
\hline Final $R$ indices $[\mathrm{I}>2 \operatorname{sigma}(\mathrm{I})]$ & \multicolumn{2}{|c|}{$\mathrm{R} 1=0.0297, \mathrm{wR} 2=0.0816$} \\
\hline $\mathrm{R}$ indices (all data) & \multicolumn{2}{|c|}{$\mathrm{R} 1=0.0327, \mathrm{wR} 2=0.0832$} \\
\hline Largest diff. peak and hole & \multicolumn{2}{|c|}{0.753 and -0.417 e. $\AA^{-3}$} \\
\hline
\end{tabular}


Table S9. Atomic coordinates $\left(\times 10^{4}\right)$ and equivalent isotropic displacement parameters $\left(\AA^{2} \times 10^{3}\right)$ for (3). $U(e q)$ is defined as one third of the trace of the orthogonalized $U^{i j}$ tensor.

\begin{tabular}{|c|c|c|c|c|}
\hline & $\mathrm{x}$ & $\mathrm{y}$ & $\mathrm{Z}$ & $\mathrm{U}(\mathrm{eq})$ \\
\hline$\overline{\mathrm{Pd}}$ & $765(1)$ & $8860(1)$ & $2300(1)$ & $14(1)$ \\
\hline $\mathrm{O}(1)$ & 1388(1) & $9620(1)$ & $2995(1)$ & $24(1)$ \\
\hline $\mathrm{O}(2)$ & $657(1)$ & 10142(1) & $2603(1)$ & $22(1)$ \\
\hline $\mathrm{N}(1)$ & 2198(1) & 7172(1) & $2245(1)$ & 19(1) \\
\hline $\mathrm{N}(2)$ & $1052(1)$ & $6947(1)$ & 2795(1) & 19(1) \\
\hline $\mathrm{N}(3)$ & $-1248(1)$ & 8719(1) & 1431(1) & $24(1)$ \\
\hline $\mathrm{N}(4)$ & $-47(1)$ & 9294(1) & $1028(1)$ & $21(1)$ \\
\hline$C(1)$ & 1359(1) & $7578(1)$ & 2414(1) & $18(1)$ \\
\hline$C(2)$ & 2393(1) & $6320(1)$ & $2510(1)$ & $22(1)$ \\
\hline$C(3)$ & $1670(1)$ & $6180(1)$ & $2852(1)$ & $21(1)$ \\
\hline C(4) & 2871(1) & $7526(1)$ & $1856(1)$ & $21(1)$ \\
\hline$C(5)$ & 2957(1) & 7027(1) & $1336(1)$ & $25(1)$ \\
\hline$C(6)$ & $3702(1)$ & 7291(1) & $1003(1)$ & $30(1)$ \\
\hline$C(7)$ & $4344(1)$ & $8018(1)$ & $1166(1)$ & $32(1)$ \\
\hline $\mathrm{C}(8)$ & $4234(1)$ & $8498(1)$ & $1684(1)$ & $29(1)$ \\
\hline$C(9)$ & $3512(1)$ & $8249(1)$ & 2044(1) & $23(1)$ \\
\hline$C(10)$ & 2301(2) & $6207(1)$ & $1153(1)$ & $32(1)$ \\
\hline$C(11)$ & $5152(2)$ & $8274(2)$ & $793(1)$ & $46(1)$ \\
\hline$C(12)$ & $3483(1)$ & $8723(1)$ & $2632(1)$ & $27(1)$ \\
\hline$C(13)$ & $225(1)$ & 7041(1) & $3132(1)$ & 21(1) \\
\hline$C(14)$ & $352(1)$ & $7550(1)$ & $3657(1)$ & $25(1)$ \\
\hline$C(15)$ & $-460(2)$ & $7588(1)$ & $3980(1)$ & $32(1)$ \\
\hline$C(16)$ & $-1356(2)$ & 7124(1) & 3799(1) & $33(1)$ \\
\hline$C(17)$ & $-1432(1)$ & $6590(1)$ & $3289(1)$ & $32(1)$ \\
\hline$C(18)$ & $-650(1)$ & 6534(1) & 2943(1) & $24(1)$ \\
\hline$C(19)$ & $1325(2)$ & $8034(1)$ & $3884(1)$ & $29(1)$ \\
\hline$C(20)$ & $-2237(2)$ & $7221(2)$ & $4143(1)$ & $50(1)$ \\
\hline$C(21)$ & $-728(1)$ & 5944(1) & 2396(1) & $32(1)$ \\
\hline$C(22)$ & $-225(1)$ & $8857(1)$ & $1535(1)$ & $17(1)$ \\
\hline$C(23)$ & $-1670(1)$ & $9071(2)$ & $883(1)$ & $36(1)$ \\
\hline$C(24)$ & $-919(1)$ & $9429(2)$ & 631(1) & $33(1)$ \\
\hline$C(25)$ & $-1897(1)$ & $8268(1)$ & $1797(1)$ & $21(1)$ \\
\hline$C(26)$ & $-2181(1)$ & $8729(1)$ & $2290(1)$ & $23(1)$ \\
\hline$C(27)$ & $-2933(1)$ & $8326(1)$ & $2570(1)$ & $24(1)$ \\
\hline$C(28)$ & $-3395(1)$ & 7512(1) & $2378(1)$ & $27(1)$ \\
\hline$C(29)$ & $-3086(1)$ & 7079(1) & 1892(1) & $29(1)$ \\
\hline$C(30)$ & $-2337(1)$ & $7445(1)$ & 1592(1) & $27(1)$ \\
\hline$C(31)$ & $-1713(2)$ & $9624(1)$ & 2497(1) & $37(1)$ \\
\hline$C(32)$ & $-4205(1)$ & 7093(1) & 2694(1) & $34(1)$ \\
\hline C(33) & $-2036(2)$ & 6958(2) & $1058(1)$ & $42(1)$ \\
\hline$C(34)$ & $923(1)$ & 9591(1) & $894(1)$ & $18(1)$ \\
\hline$C(35)$ & $1210(1)$ & 10499(1) & $1000(1)$ & $20(1)$ \\
\hline$C(36)$ & $2125(1)$ & $10776(1)$ & $825(1)$ & $22(1)$ \\
\hline C(37) & $2735(1)$ & $10173(1)$ & $566(1)$ & $21(1)$ \\
\hline C(38) & $2408(1)$ & $9280(1)$ & $458(1)$ & $20(1)$ \\
\hline C(39) & $1490(1)$ & $8976(1)$ & $606(1)$ & 19(1) \\
\hline $\mathrm{C}(40)$ & $556(2)$ & $11155(1)$ & $1282(1)$ & $29(1)$ \\
\hline $\mathrm{C}(41)$ & $3739(1)$ & 10489(1) & $409(1)$ & $31(1)$ \\
\hline$C(42)$ & 1088(1) & $8038(1)$ & $443(1)$ & $28(1)$ \\
\hline
\end{tabular}


Table S10. Bond lengths $[\AA]$ and angles $\left[{ }^{\circ}\right]$ for (3).

\begin{tabular}{llll}
\hline $\mathrm{Pd}-\mathrm{O}(1)$ & $2.0104(11)$ & $\mathrm{C}(13)-\mathrm{C}(18)$ & $1.402(2)$ \\
$\mathrm{Pd}-\mathrm{O}(2)$ & $2.0104(12)$ & $\mathrm{C}(14)-\mathrm{C}(15)$ & $1.397(3)$ \\
$\mathrm{Pd}-\mathrm{C}(22)$ & $2.0266(17)$ & $\mathrm{C}(14)-\mathrm{C}(19)$ & $1.508(3)$ \\
$\mathrm{Pd}-\mathrm{C}(1)$ & $2.0414(16)$ & $\mathrm{C}(15)-\mathrm{C}(16)$ & $1.389(3)$ \\
$\mathrm{O}(1)-\mathrm{O}(2)$ & $\mathrm{C}(16)-\mathrm{C}(17)$ & $1.386(3)$ \\
$\mathrm{N}(1)-\mathrm{C}(1)$ & $\mathrm{C}(16)-\mathrm{C}(20)$ & $1.512(3)$ \\
$\mathrm{N}(1)-\mathrm{C}(2)$ & $1.4429(16)$ & $\mathrm{C}(17)-\mathrm{C}(18)$ & $1.397(2)$ \\
$\mathrm{N}(1)-\mathrm{C}(4)$ & $1.373(2)$ & $\mathrm{C}(18)-\mathrm{C}(21)$ & $1.505(3)$ \\
$\mathrm{N}(2)-\mathrm{C}(1)$ & $\mathrm{C}(23)-\mathrm{C}(24)$ & $1.333(3)$ \\
$\mathrm{N}(2)-\mathrm{C}(3)$ & $\mathrm{C}(25)-\mathrm{C}(30)$ & $1.391(3)$ \\
$\mathrm{N}(2)-\mathrm{C}(13)$ & $1.443(2)$ & $\mathrm{C}(25)-\mathrm{C}(26)$ & $1.405(3)$ \\
$\mathrm{N}(3)-\mathrm{C}(22)$ & $1.367(2)$ & $\mathrm{C}(26)-\mathrm{C}(27)$ & $1.396(2)$ \\
$\mathrm{N}(3)-\mathrm{C}(23)$ & $1.390(2)$ & $\mathrm{C}(26)-\mathrm{C}(31)$ & $1.499(3)$ \\
$\mathrm{N}(3)-\mathrm{C}(25)$ & $\mathrm{C}(27)-\mathrm{C}(28)$ & $1.384(3)$ \\
$\mathrm{N}(4)-\mathrm{C}(22)$ & $\mathrm{C}(28)-\mathrm{C}(29)$ & $1.383(3)$ \\
$\mathrm{N}(4)-\mathrm{C}(24)$ & $\mathrm{C}(28)-\mathrm{C}(32)$ & $1.512(2)$ \\
$\mathrm{N}(4)-\mathrm{C}(34)$ & $1.438(2)$ & $\mathrm{C}(29)-\mathrm{C}(30)$ & $1.397(2)$ \\
$\mathrm{C}(2)-\mathrm{C}(3)$ & $1.371(2)$ & $\mathrm{C}(30)-\mathrm{C}(33)$ & $1.510(3)$ \\
$\mathrm{C}(4)-\mathrm{C}(9)$ & $\mathrm{C}(34)-\mathrm{C}(35)$ & $1.394(2)$ \\
$\mathrm{C}(4)-\mathrm{C}(5)$ & $1.444(2)$ & $\mathrm{C}(34)-\mathrm{C}(39)$ & $1.397(2)$ \\
$\mathrm{C}(5)-\mathrm{C}(6)$ & $1.367(2)$ & $\mathrm{C}(35)-\mathrm{C}(36)$ & $1.399(2)$ \\
$\mathrm{C}(5)-\mathrm{C}(10)$ & $1.383(2)$ & $\mathrm{C}(35)-\mathrm{C}(40)$ & $1.503(2)$ \\
$\mathrm{C}(6)-\mathrm{C}(7)$ & $1.442(2)$ & $\mathrm{C}(36)-\mathrm{C}(37)$ & $1.389(2)$ \\
$\mathrm{C}(7)-\mathrm{C}(8)$ & $1.339(2)$ & $\mathrm{C}(37)-\mathrm{C}(38)$ & $1.390(2)$ \\
$\mathrm{C}(7)-\mathrm{C}(11)$ & $\mathrm{C}(37)-\mathrm{C}(41)$ & $1.512(2)$ \\
$\mathrm{C}(8)-\mathrm{C}(9)$ & $\mathrm{C}(38)-\mathrm{C}(39)$ & $1.394(2)$ \\
$\mathrm{C}(9)-\mathrm{C}(12)$ & $\mathrm{C}(39)-\mathrm{C}(42)$ & $1.503(2)$ \\
$\mathrm{C}(13)-\mathrm{C}(14)$ & &
\end{tabular}




\begin{tabular}{|c|c|}
\hline $\mathrm{O}(1)-\mathrm{Pd}-\mathrm{O}(2)$ & $42.06(5)$ \\
\hline $\mathrm{O}(1)-\mathrm{Pd}-\mathrm{C}(22)$ & $145.06(5)$ \\
\hline $\mathrm{O}(2)-\mathrm{Pd}-\mathrm{C}(22)$ & $103.01(5)$ \\
\hline $\mathrm{O}(1)-\mathrm{Pd}-\mathrm{C}(1)$ & $107.36(5)$ \\
\hline $\mathrm{O}(2)-\mathrm{Pd}-\mathrm{C}(1)$ & $149.37(5)$ \\
\hline $\mathrm{C}(22)-\mathrm{Pd}-\mathrm{C}(1)$ & $107.58(6)$ \\
\hline $\mathrm{O}(2)-\mathrm{O}(1)-\mathrm{Pd}$ & $68.97(6)$ \\
\hline $\mathrm{O}(1)-\mathrm{O}(2)-\mathrm{Pd}$ & $68.97(6)$ \\
\hline $\mathrm{C}(1)-\mathrm{N}(1)-\mathrm{C}(2)$ & $112.14(13)$ \\
\hline $\mathrm{C}(1)-\mathrm{N}(1)-\mathrm{C}(4)$ & $128.47(14)$ \\
\hline $\mathrm{C}(2)-\mathrm{N}(1)-\mathrm{C}(4)$ & $119.37(13)$ \\
\hline $\mathrm{C}(1)-\mathrm{N}(2)-\mathrm{C}(3)$ & $112.07(14)$ \\
\hline $\mathrm{C}(1)-\mathrm{N}(2)-\mathrm{C}(13)$ & $126.41(13)$ \\
\hline $\mathrm{C}(3)-\mathrm{N}(2)-\mathrm{C}(13)$ & $121.46(13)$ \\
\hline $\mathrm{C}(22)-\mathrm{N}(3)-\mathrm{C}(23)$ & $111.66(14)$ \\
\hline $\mathrm{C}(22)-\mathrm{N}(3)-\mathrm{C}(25)$ & $129.48(14)$ \\
\hline $\mathrm{C}(23)-\mathrm{N}(3)-\mathrm{C}(25)$ & $118.86(14)$ \\
\hline $\mathrm{C}(22)-\mathrm{N}(4)-\mathrm{C}(24)$ & $112.45(14)$ \\
\hline $\mathrm{C}(22)-\mathrm{N}(4)-\mathrm{C}(34)$ & $126.11(13)$ \\
\hline $\mathrm{C}(24)-\mathrm{N}(4)-\mathrm{C}(34)$ & $121.44(13)$ \\
\hline $\mathrm{N}(2)-\mathrm{C}(1)-\mathrm{N}(1)$ & $102.46(13)$ \\
\hline $\mathrm{N}(2)-\mathrm{C}(1)-\mathrm{Pd}$ & $123.71(11)$ \\
\hline $\mathrm{N}(1)-\mathrm{C}(1)-\mathrm{Pd}$ & $133.02(12)$ \\
\hline $\mathrm{C}(3)-\mathrm{C}(2)-\mathrm{N}(1)$ & $106.31(14)$ \\
\hline $\mathrm{C}(2)-\mathrm{C}(3)-\mathrm{N}(2)$ & $107.02(14)$ \\
\hline$C(9)-C(4)-C(5)$ & $122.16(16)$ \\
\hline $\mathrm{C}(9)-\mathrm{C}(4)-\mathrm{N}(1)$ & $119.86(15)$ \\
\hline $\mathrm{C}(5)-\mathrm{C}(4)-\mathrm{N}(1)$ & $117.25(15)$ \\
\hline $\mathrm{C}(6)-\mathrm{C}(5)-\mathrm{C}(4)$ & $117.50(17)$ \\
\hline$C(6)-C(5)-C(10)$ & $120.45(17)$ \\
\hline $\mathrm{C}(4)-\mathrm{C}(5)-\mathrm{C}(10)$ & $122.00(16)$ \\
\hline$C(7)-C(6)-C(5)$ & $122.36(18)$ \\
\hline $\mathrm{C}(6)-\mathrm{C}(7)-\mathrm{C}(8)$ & $118.48(17)$ \\
\hline$C(6)-C(7)-C(11)$ & $120.48(19)$ \\
\hline $\mathrm{C}(8)-\mathrm{C}(7)-\mathrm{C}(11)$ & $121.0(2)$ \\
\hline $\mathrm{C}(7)-\mathrm{C}(8)-\mathrm{C}(9)$ & $121.65(18)$ \\
\hline $\mathrm{C}(4)-\mathrm{C}(9)-\mathrm{C}(8)$ & $117.79(17)$ \\
\hline$C(4)-C(9)-C(12)$ & $121.92(15)$ \\
\hline $\mathrm{C}(8)-\mathrm{C}(9)-\mathrm{C}(12)$ & $120.19(16)$ \\
\hline $\mathrm{C}(14)-\mathrm{C}(13)-\mathrm{C}(18)$ & $122.56(16)$ \\
\hline $\mathrm{C}(14)-\mathrm{C}(13)-\mathrm{N}(2)$ & $119.68(15)$ \\
\hline $\mathrm{C}(18)-\mathrm{C}(13)-\mathrm{N}(2)$ & $117.43(15)$ \\
\hline$C(13)-C(14)-C(15)$ & $117.41(17)$ \\
\hline $\mathrm{C}(13)-\mathrm{C}(14)-\mathrm{C}(19)$ & $122.49(16)$ \\
\hline $\mathrm{C}(15)-\mathrm{C}(14)-\mathrm{C}(19)$ & $120.09(17)$ \\
\hline$C(16)-C(15)-C(14)$ & 121.91(18) \\
\hline $\mathrm{C}(17)-\mathrm{C}(16)-\mathrm{C}(15)$ & $118.76(17)$ \\
\hline$C(17)-C(16)-C(20)$ & $120.9(2)$ \\
\hline $\mathrm{C}(15)-\mathrm{C}(16)-\mathrm{C}(20)$ & $120.3(2)$ \\
\hline $\mathrm{C}(16)-\mathrm{C}(17)-\mathrm{C}(18)$ & 121.91(18) \\
\hline $\mathrm{C}(17)-\mathrm{C}(18)-\mathrm{C}(13)$ & $117.31(17)$ \\
\hline $\mathrm{C}(17)-\mathrm{C}(18)-\mathrm{C}(21)$ & $121.87(17)$ \\
\hline $\mathrm{C}(13)-\mathrm{C}(18)-\mathrm{C}(21)$ & $120.82(16)$ \\
\hline $\mathrm{N}(4)-\mathrm{C}(22)-\mathrm{N}(3)$ & $102.35(14)$ \\
\hline $\mathrm{N}(4)-\mathrm{C}(22)-\mathrm{Pd}$ & $123.16(12)$ \\
\hline $\mathrm{N}(3)-\mathrm{C}(22)-\mathrm{Pd}$ & $131.58(12)$ \\
\hline $\mathrm{C}(24)-\mathrm{C}(23)-\mathrm{N}(3)$ & $107.08(16)$ \\
\hline $\mathrm{C}(23)-\mathrm{C}(24)-\mathrm{N}(4)$ & $106.47(15)$ \\
\hline
\end{tabular}




$\begin{array}{ll}\text { C(30)-C(25)-C(26) } & 122.14(15) \\ \text { C(30)-C(25)-N(3) } & 117.70(15) \\ \text { C(26)-C(25)-N(3) } & 119.44(16) \\ \text { C(27)-C(26)-C(25) } & 116.97(16) \\ \text { C(27)-C(26)-C(31) } & 121.64(17) \\ \text { C(25)-C(26)-C(31) } & 121.38(16) \\ \text { C(28)-C(27)-C(26) } & 122.67(17) \\ \text { C(29)-C(28)-C(27) } & 118.34(16) \\ \text { C(29)-C(28)-C(32) } & 120.48(18) \\ \text { C(27)-C(28)-C(32) } & 121.17(17) \\ \text { C(28)-C(29)-C(30) } & 121.88(17) \\ \text { C(25)-C(30)-C(29) } & 118.01(17) \\ \text { C(25)-C(30)-C(33) } & 121.86(16) \\ \text { C(29)-C(30)-C(33) } & 120.12(18) \\ \text { C(35)-C(34)-C(39) } & 122.64(15) \\ \text { C(35)-C(34)-N(4) } & 119.03(14) \\ \text { C(39)-C(34)-N(4) } & 117.99(15) \\ \text { C(34)-C(35)-C(36) } & 117.28(15) \\ \text { C(34)-C(35)-C(40) } & 121.29(15) \\ \text { C(36)-C(35)-C(40) } & 121.42(16) \\ \text { C(37)-C(36)-C(35) } & 121.81(16) \\ \text { C(36)-C(37)-C(38) } & 118.89(15) \\ \text { C(36)-C(37)-C(41) } & 120.16(16) \\ \text { C(38)-C(37)-C(41) } & 120.96(16) \\ \text { C(37)-C(38)-C(39) } & 121.58(15) \\ \text { C(38)-C(39)-C(34) } & 117.66(15) \\ \text { C(38)-C(39)-C(42) } & 121.93(15) \\ \text { C(34)-C(39)-C(42) } & 120.36(15)\end{array}$

Symmetry transformations used to generate equivalent atoms: 
Table S11. Anisotropic displacement parameters $\left(\AA^{2} \times 10^{3}\right)$ for (3). The anisotropic displacement factor exponent takes the form: $-2 \pi^{2}\left[h^{2} a^{* 2} U^{11}+\ldots+2 h k a^{*} b^{*} U^{12}\right]$

\begin{tabular}{|c|c|c|c|c|c|c|}
\hline & $\mathrm{U}^{11}$ & $\mathrm{U}^{22}$ & $\mathrm{U}^{33}$ & $\mathrm{U}^{23}$ & $\mathrm{U}^{13}$ & $\mathrm{U}^{12}$ \\
\hline $\mathrm{Pd}$ & $13(1)$ & $15(1)$ & $15(1)$ & $2(1)$ & $3(1)$ & 1(1) \\
\hline $\mathrm{O}(1)$ & $26(1)$ & $21(1)$ & $22(1)$ & $-1(1)$ & $-2(1)$ & 1(1) \\
\hline $\mathrm{O}(2)$ & $23(1)$ & $17(1)$ & $27(1)$ & 1(1) & 2(1) & $3(1)$ \\
\hline $\mathrm{N}(1)$ & $17(1)$ & $18(1)$ & $21(1)$ & $2(1)$ & $4(1)$ & $3(1)$ \\
\hline $\mathrm{N}(2)$ & $19(1)$ & $18(1)$ & $19(1)$ & $2(1)$ & $3(1)$ & $1(1)$ \\
\hline$N(3)$ & $16(1)$ & $35(1)$ & $22(1)$ & $10(1)$ & $3(1)$ & $-3(1)$ \\
\hline $\mathrm{N}(4)$ & $15(1)$ & $28(1)$ & $19(1)$ & $7(1)$ & $3(1)$ & $-1(1)$ \\
\hline $\mathrm{C}(1)$ & $17(1)$ & $20(1)$ & $16(1)$ & $-1(1)$ & 1(1) & $-1(1)$ \\
\hline $\mathrm{C}(2)$ & $22(1)$ & 19(1) & $25(1)$ & $3(1)$ & $3(1)$ & $5(1)$ \\
\hline$C(3)$ & $23(1)$ & $17(1)$ & $23(1)$ & $4(1)$ & $2(1)$ & $3(1)$ \\
\hline C(4) & $17(1)$ & $22(1)$ & $23(1)$ & $7(1)$ & $4(1)$ & $6(1)$ \\
\hline$C(5)$ & $28(1)$ & $24(1)$ & $24(1)$ & $5(1)$ & $5(1)$ & $10(1)$ \\
\hline$C(6)$ & $35(1)$ & $34(1)$ & $24(1)$ & $7(1)$ & $11(1)$ & $14(1)$ \\
\hline$C(7)$ & $24(1)$ & $43(1)$ & $32(1)$ & $18(1)$ & $12(1)$ & $13(1)$ \\
\hline C(8) & $18(1)$ & $32(1)$ & $37(1)$ & $13(1)$ & $4(1)$ & $3(1)$ \\
\hline $\mathrm{C}(9)$ & $16(1)$ & $26(1)$ & $28(1)$ & $8(1)$ & $3(1)$ & $5(1)$ \\
\hline $\mathrm{C}(10)$ & $45(1)$ & $27(1)$ & $22(1)$ & $0(1)$ & $4(1)$ & $6(1)$ \\
\hline$C(11)$ & $33(1)$ & $61(2)$ & $46(1)$ & $22(1)$ & $20(1)$ & $10(1)$ \\
\hline$C(12)$ & $22(1)$ & $25(1)$ & $35(1)$ & 1(1) & $4(1)$ & $-4(1)$ \\
\hline $\mathrm{C}(13)$ & $22(1)$ & 19(1) & $22(1)$ & $7(1)$ & $7(1)$ & $4(1)$ \\
\hline$C(14)$ & $33(1)$ & $21(1)$ & $21(1)$ & $7(1)$ & $5(1)$ & $7(1)$ \\
\hline$C(15)$ & $43(1)$ & $31(1)$ & $23(1)$ & $8(1)$ & $11(1)$ & 11(1) \\
\hline$C(16)$ & $34(1)$ & $39(1)$ & $28(1)$ & $16(1)$ & $14(1)$ & $12(1)$ \\
\hline$C(17)$ & $21(1)$ & $36(1)$ & $41(1)$ & $16(1)$ & $8(1)$ & $2(1)$ \\
\hline $\mathrm{C}(18)$ & $20(1)$ & $24(1)$ & $29(1)$ & $7(1)$ & $5(1)$ & $3(1)$ \\
\hline$C(19)$ & $40(1)$ & $24(1)$ & $23(1)$ & $2(1)$ & $5(1)$ & $2(1)$ \\
\hline$C(20)$ & $42(1)$ & $72(2)$ & $40(1)$ & 19(1) & $23(1)$ & $16(1)$ \\
\hline$C(21)$ & $22(1)$ & $33(1)$ & $41(1)$ & $-3(1)$ & $3(1)$ & $-3(1)$ \\
\hline $\mathrm{C}(22)$ & $17(1)$ & $18(1)$ & $18(1)$ & $2(1)$ & $5(1)$ & $0(1)$ \\
\hline$C(23)$ & $17(1)$ & $60(1)$ & $28(1)$ & 19(1) & $-4(1)$ & $-5(1)$ \\
\hline$C(24)$ & $19(1)$ & $54(1)$ & $23(1)$ & 19(1) & $-2(1)$ & $-5(1)$ \\
\hline$C(25)$ & $12(1)$ & $27(1)$ & $23(1)$ & $10(1)$ & $3(1)$ & $-1(1)$ \\
\hline$C(26)$ & $16(1)$ & $22(1)$ & $31(1)$ & $6(1)$ & $4(1)$ & $3(1)$ \\
\hline$C(27)$ & $19(1)$ & $28(1)$ & $27(1)$ & $6(1)$ & $8(1)$ & $4(1)$ \\
\hline $\mathrm{C}(28)$ & $18(1)$ & $28(1)$ & $35(1)$ & $15(1)$ & $8(1)$ & 1(1) \\
\hline$C(29)$ & $21(1)$ & $28(1)$ & $38(1)$ & 1(1) & $5(1)$ & $-6(1)$ \\
\hline$C(30)$ & $21(1)$ & $34(1)$ & $25(1)$ & 1(1) & $3(1)$ & $-1(1)$ \\
\hline $\mathrm{C}(31)$ & $26(1)$ & $28(1)$ & $59(1)$ & $-9(1)$ & $17(1)$ & $-1(1)$ \\
\hline $\mathrm{C}(32)$ & $23(1)$ & $34(1)$ & $48(1)$ & $18(1)$ & $15(1)$ & $0(1)$ \\
\hline$C(33)$ & $37(1)$ & $54(1)$ & $37(1)$ & $-14(1)$ & 11(1) & $-11(1)$ \\
\hline $\mathrm{C}(34)$ & $15(1)$ & $25(1)$ & $14(1)$ & $6(1)$ & $2(1)$ & $0(1)$ \\
\hline$C(35)$ & $22(1)$ & $23(1)$ & $16(1)$ & $2(1)$ & $5(1)$ & $2(1)$ \\
\hline C(36) & $24(1)$ & $22(1)$ & 19(1) & $0(1)$ & $3(1)$ & $-4(1)$ \\
\hline $\mathrm{C}(37)$ & $17(1)$ & $31(1)$ & $16(1)$ & $4(1)$ & $3(1)$ & $-3(1)$ \\
\hline $\mathrm{C}(38)$ & $18(1)$ & $25(1)$ & $17(1)$ & 1(1) & $3(1)$ & $5(1)$ \\
\hline C(39) & $20(1)$ & $22(1)$ & $15(1)$ & $4(1)$ & 1(1) & $2(1)$ \\
\hline$C(40)$ & $34(1)$ & $27(1)$ & $29(1)$ & $-1(1)$ & $12(1)$ & $4(1)$ \\
\hline $\mathrm{C}(41)$ & $21(1)$ & $44(1)$ & $29(1)$ & $0(1)$ & $8(1)$ & $-8(1)$ \\
\hline $\mathrm{C}(42)$ & $30(1)$ & $21(1)$ & $33(1)$ & $2(1)$ & $2(1)$ & $-1(1)$ \\
\hline
\end{tabular}


Table S12. Hydrogen coordinates ( $\left.\times 10^{4}\right)$ and isotropic displacement parameters $\left(\AA^{2} \times 10^{3}\right)$ for (3).

\begin{tabular}{|c|c|c|c|c|}
\hline & $\mathrm{x}$ & $\mathrm{y}$ & $\mathrm{Z}$ & $\mathrm{U}(\mathrm{eq})$ \\
\hline $\mathrm{H}(2)$ & 2931 & 5919 & 2459 & 26 \\
\hline $\mathrm{H}(3)$ & 1593 & 5655 & 3088 & 25 \\
\hline $\mathrm{H}(6)$ & 3774 & 6962 & 650 & 36 \\
\hline $\mathrm{H}(8)$ & 4660 & 9006 & 1795 & 35 \\
\hline $\mathrm{H}(10 \mathrm{~A})$ & 2252 & 6119 & 722 & 47 \\
\hline $\mathrm{H}(10 \mathrm{~B})$ & 1626 & 6308 & 1260 & 47 \\
\hline $\mathrm{H}(10 \mathrm{C})$ & 2600 & 5663 & 1359 & 47 \\
\hline $\mathrm{H}(11 \mathrm{~A})$ & 4835 & 8534 & 414 & 68 \\
\hline $\mathrm{H}(11 \mathrm{~B})$ & 5537 & 7728 & 716 & 68 \\
\hline $\mathrm{H}(11 \mathrm{C})$ & 5606 & 8726 & 1008 & 68 \\
\hline $\mathrm{H}(12 \mathrm{~A})$ & 2891 & 9123 & 2601 & 41 \\
\hline $\mathrm{H}(12 \mathrm{~B})$ & 4097 & 9088 & 2734 & 41 \\
\hline $\mathrm{H}(12 \mathrm{C})$ & 3442 & 8265 & 2944 & 41 \\
\hline $\mathrm{H}(15)$ & -398 & 7942 & 4335 & 38 \\
\hline $\mathrm{H}(17)$ & -2034 & 6253 & 3172 & 39 \\
\hline $\mathrm{H}(19 \mathrm{~A})$ & 1886 & 7730 & 3730 & 44 \\
\hline $\mathrm{H}(19 \mathrm{~B})$ & 1437 & 8018 & 4320 & 44 \\
\hline $\mathrm{H}(19 \mathrm{C})$ & 1284 & 8671 & 3748 & 44 \\
\hline $\mathrm{H}(20 \mathrm{~A})$ & -1995 & 7441 & 4546 & 74 \\
\hline $\mathrm{H}(20 \mathrm{~B})$ & -2566 & 6627 & 4166 & 74 \\
\hline $\mathrm{H}(20 \mathrm{C})$ & -2723 & 7659 & 3940 & 74 \\
\hline $\mathrm{H}(21 \mathrm{~A})$ & -315 & 5394 & 2483 & 48 \\
\hline $\mathrm{H}(21 \mathrm{~B})$ & -485 & 6285 & 2072 & 48 \\
\hline $\mathrm{H}(21 \mathrm{C})$ & -1433 & 5767 & 2274 & 48 \\
\hline $\mathrm{H}(23)$ & -2363 & 9059 & 719 & 43 \\
\hline $\mathrm{H}(24)$ & -972 & 9720 & 254 & 39 \\
\hline $\mathrm{H}(27)$ & -3136 & 8622 & 2906 & 29 \\
\hline $\mathrm{H}(29)$ & -3392 & 6516 & 1759 & 35 \\
\hline $\mathrm{H}(31 \mathrm{~A})$ & -2094 & 9897 & 2790 & 55 \\
\hline $\mathrm{H}(31 \mathrm{~B})$ & -1725 & 10037 & 2156 & 55 \\
\hline $\mathrm{H}(31 \mathrm{C})$ & -1013 & 9524 & 2680 & 55 \\
\hline $\mathrm{H}(32 \mathrm{~A})$ & -4668 & 6735 & 2409 & 51 \\
\hline $\mathrm{H}(32 \mathrm{~B})$ & -4580 & 7579 & 2862 & 51 \\
\hline $\mathrm{H}(32 \mathrm{C})$ & -3891 & 6694 & 3014 & 51 \\
\hline $\mathrm{H}(33 \mathrm{~A})$ & -2409 & 7217 & 693 & 63 \\
\hline $\mathrm{H}(33 \mathrm{~B})$ & -2194 & 6307 & 1080 & 63 \\
\hline $\mathrm{H}(33 \mathrm{C})$ & -1310 & 7035 & 1053 & 63 \\
\hline $\mathrm{H}(36)$ & 2333 & 11393 & 886 & 26 \\
\hline $\mathrm{H}(38)$ & 2821 & 8866 & 278 & 24 \\
\hline $\mathrm{H}(40 \mathrm{~A})$ & -105 & 11201 & 1035 & 44 \\
\hline $\mathrm{H}(40 \mathrm{~B})$ & 877 & 11758 & 1316 & 44 \\
\hline $\mathrm{H}(40 \mathrm{C})$ & 470 & 10934 & 1680 & 44 \\
\hline $\mathrm{H}(41 \mathrm{~A})$ & 3683 & 11126 & 274 & 46 \\
\hline $\mathrm{H}(41 \mathrm{~B})$ & 3931 & 10104 & 90 & 46 \\
\hline $\mathrm{H}(41 \mathrm{C})$ & 4255 & 10443 & 761 & 46 \\
\hline $\mathrm{H}(42 \mathrm{~A})$ & 1593 & 7691 & 262 & 42 \\
\hline $\mathrm{H}(42 \mathrm{~B})$ & 468 & 8088 & 157 & 42 \\
\hline $\mathrm{H}(42 \mathrm{C})$ & 942 & 7722 & 802 & 42 \\
\hline
\end{tabular}


Table S13. Torsion angles $\left[^{\circ}\right]$ for (3).

\begin{tabular}{|c|c|}
\hline$\overline{\mathrm{C}(22)-\mathrm{Pd}-\mathrm{O}(1)-\mathrm{O}(2)}$ & $1.51(13)$ \\
\hline $\mathrm{C}(1)-\mathrm{Pd}-\mathrm{O}(1)-\mathrm{O}(2)$ & $-177.77(7)$ \\
\hline $\mathrm{C}(22)-\mathrm{Pd}-\mathrm{O}(2)-\mathrm{O}(1)$ & $-179.11(8)$ \\
\hline $\mathrm{C}(1)-\mathrm{Pd}-\mathrm{O}(2)-\mathrm{O}(1)$ & $4.18(14)$ \\
\hline $\mathrm{C}(3)-\mathrm{N}(2)-\mathrm{C}(1)-\mathrm{N}(1)$ & $-0.68(17)$ \\
\hline $\mathrm{C}(13)-\mathrm{N}(2)-\mathrm{C}(1)-\mathrm{N}(1)$ & $176.54(14)$ \\
\hline $\mathrm{C}(3)-\mathrm{N}(2)-\mathrm{C}(1)-\mathrm{Pd}$ & $-171.55(11)$ \\
\hline $\mathrm{C}(13)-\mathrm{N}(2)-\mathrm{C}(1)-\mathrm{Pd}$ & $5.7(2)$ \\
\hline $\mathrm{C}(2)-\mathrm{N}(1)-\mathrm{C}(1)-\mathrm{N}(2)$ & $0.30(17)$ \\
\hline $\mathrm{C}(4)-\mathrm{N}(1)-\mathrm{C}(1)-\mathrm{N}(2)$ & $-177.98(15)$ \\
\hline $\mathrm{C}(2)-\mathrm{N}(1)-\mathrm{C}(1)-\mathrm{Pd}$ & $169.90(12)$ \\
\hline $\mathrm{C}(4)-\mathrm{N}(1)-\mathrm{C}(1)-\mathrm{Pd}$ & $-8.4(2)$ \\
\hline $\mathrm{O}(1)-\mathrm{Pd}-\mathrm{C}(1)-\mathrm{N}(2)$ & $78.53(13)$ \\
\hline $\mathrm{O}(2)-\mathrm{Pd}-\mathrm{C}(1)-\mathrm{N}(2)$ & $75.60(17)$ \\
\hline $\mathrm{C}(22)-\mathrm{Pd}-\mathrm{C}(1)-\mathrm{N}(2)$ & $-101.03(13)$ \\
\hline $\mathrm{O}(1)-\mathrm{Pd}-\mathrm{C}(1)-\mathrm{N}(1)$ & $-89.24(15)$ \\
\hline $\mathrm{O}(2)-\mathrm{Pd}-\mathrm{C}(1)-\mathrm{N}(1)$ & $-92.17(18)$ \\
\hline $\mathrm{C}(22)-\mathrm{Pd}-\mathrm{C}(1)-\mathrm{N}(1)$ & $91.19(16)$ \\
\hline $\mathrm{C}(1)-\mathrm{N}(1)-\mathrm{C}(2)-\mathrm{C}(3)$ & $0.18(19)$ \\
\hline $\mathrm{C}(4)-\mathrm{N}(1)-\mathrm{C}(2)-\mathrm{C}(3)$ & $178.64(14)$ \\
\hline $\mathrm{N}(1)-\mathrm{C}(2)-\mathrm{C}(3)-\mathrm{N}(2)$ & $-0.58(19)$ \\
\hline $\mathrm{C}(1)-\mathrm{N}(2)-\mathrm{C}(3)-\mathrm{C}(2)$ & $0.82(19)$ \\
\hline$C(13)-N(2)-C(3)-C(2)$ & $-176.55(15)$ \\
\hline $\mathrm{C}(1)-\mathrm{N}(1)-\mathrm{C}(4)-\mathrm{C}(9)$ & $70.0(2)$ \\
\hline $\mathrm{C}(2)-\mathrm{N}(1)-\mathrm{C}(4)-\mathrm{C}(9)$ & $-108.13(18)$ \\
\hline $\mathrm{C}(1)-\mathrm{N}(1)-\mathrm{C}(4)-\mathrm{C}(5)$ & $-119.54(18)$ \\
\hline $\mathrm{C}(2)-\mathrm{N}(1)-\mathrm{C}(4)-\mathrm{C}(5)$ & $62.3(2)$ \\
\hline$C(9)-C(4)-C(5)-C(6)$ & $-1.2(2)$ \\
\hline $\mathrm{N}(1)-\mathrm{C}(4)-\mathrm{C}(5)-\mathrm{C}(6)$ & $-171.35(14)$ \\
\hline $\mathrm{C}(9)-\mathrm{C}(4)-\mathrm{C}(5)-\mathrm{C}(10)$ & $176.26(16)$ \\
\hline $\mathrm{N}(1)-\mathrm{C}(4)-\mathrm{C}(5)-\mathrm{C}(10)$ & $6.1(2)$ \\
\hline$C(4)-C(5)-C(6)-C(7)$ & $-0.1(3)$ \\
\hline$C(10)-C(5)-C(6)-C(7)$ & $-177.61(17)$ \\
\hline$C(5)-C(6)-C(7)-C(8)$ & $0.0(3)$ \\
\hline $\mathrm{C}(5)-\mathrm{C}(6)-\mathrm{C}(7)-\mathrm{C}(11)$ & $179.34(17)$ \\
\hline $\mathrm{C}(6)-\mathrm{C}(7)-\mathrm{C}(8)-\mathrm{C}(9)$ & $1.5(3)$ \\
\hline$C(11)-C(7)-C(8)-C(9)$ & $-177.87(17)$ \\
\hline$C(5)-C(4)-C(9)-C(8)$ & $2.5(2)$ \\
\hline $\mathrm{N}(1)-\mathrm{C}(4)-\mathrm{C}(9)-\mathrm{C}(8)$ & $172.47(14)$ \\
\hline$C(5)-C(4)-C(9)-C(12)$ & $-173.90(16)$ \\
\hline $\mathrm{N}(1)-\mathrm{C}(4)-\mathrm{C}(9)-\mathrm{C}(12)$ & $-4.0(2)$ \\
\hline $\mathrm{C}(7)-\mathrm{C}(8)-\mathrm{C}(9)-\mathrm{C}(4)$ & $-2.7(3)$ \\
\hline $\mathrm{C}(7)-\mathrm{C}(8)-\mathrm{C}(9)-\mathrm{C}(12)$ & $173.81(16)$ \\
\hline $\mathrm{C}(1)-\mathrm{N}(2)-\mathrm{C}(13)-\mathrm{C}(14)$ & $-79.6(2)$ \\
\hline $\mathrm{C}(3)-\mathrm{N}(2)-\mathrm{C}(13)-\mathrm{C}(14)$ & $97.41(19)$ \\
\hline $\mathrm{C}(1)-\mathrm{N}(2)-\mathrm{C}(13)-\mathrm{C}(18)$ & $106.75(19)$ \\
\hline $\mathrm{C}(3)-\mathrm{N}(2)-\mathrm{C}(13)-\mathrm{C}(18)$ & $-76.3(2)$ \\
\hline$C(18)-C(13)-C(14)-C(15)$ & $-4.0(2)$ \\
\hline $\mathrm{N}(2)-\mathrm{C}(13)-\mathrm{C}(14)-\mathrm{C}(15)$ & $-177.33(14)$ \\
\hline$C(18)-C(13)-C(14)-C(19)$ & $175.05(16)$ \\
\hline $\mathrm{N}(2)-\mathrm{C}(13)-\mathrm{C}(14)-\mathrm{C}(19)$ & $1.7(2)$ \\
\hline$C(13)-C(14)-C(15)-C(16)$ & $1.5(3)$ \\
\hline$C(19)-C(14)-C(15)-C(16)$ & $-177.57(17)$ \\
\hline$C(14)-C(15)-C(16)-C(17)$ & $1.6(3)$ \\
\hline$C(14)-C(15)-C(16)-C(20)$ & $-176.83(17)$ \\
\hline $\mathrm{C}(15)-\mathrm{C}(16)-\mathrm{C}(17)-\mathrm{C}(18)$ & $-2.4(3)$ \\
\hline
\end{tabular}




\begin{tabular}{|c|c|}
\hline $\mathrm{C}(20)-\mathrm{C}(16)-\mathrm{C}(17)-\mathrm{C}(18)$ & $176.01(18)$ \\
\hline $\mathrm{C}(16)-\mathrm{C}(17)-\mathrm{C}(18)-\mathrm{C}(13)$ & $0.1(3)$ \\
\hline $\mathrm{C}(16)-\mathrm{C}(17)-\mathrm{C}(18)-\mathrm{C}(21)$ & $179.06(18)$ \\
\hline $\mathrm{C}(14)-\mathrm{C}(13)-\mathrm{C}(18)-\mathrm{C}(17)$ & $3.2(2)$ \\
\hline $\mathrm{N}(2)-\mathrm{C}(13)-\mathrm{C}(18)-\mathrm{C}(17)$ & $176.72(15)$ \\
\hline $\mathrm{C}(14)-\mathrm{C}(13)-\mathrm{C}(18)-\mathrm{C}(21)$ & $-175.76(17)$ \\
\hline $\mathrm{N}(2)-\mathrm{C}(13)-\mathrm{C}(18)-\mathrm{C}(21)$ & $-2.3(2)$ \\
\hline $\mathrm{C}(24)-\mathrm{N}(4)-\mathrm{C}(22)-\mathrm{N}(3)$ & $0.62(19)$ \\
\hline $\mathrm{C}(34)-\mathrm{N}(4)-\mathrm{C}(22)-\mathrm{N}(3)$ & $-178.87(15)$ \\
\hline $\mathrm{C}(24)-\mathrm{N}(4)-\mathrm{C}(22)-\mathrm{Pd}$ & $-162.05(14)$ \\
\hline $\mathrm{C}(34)-\mathrm{N}(4)-\mathrm{C}(22)-\mathrm{Pd}$ & $18.5(2)$ \\
\hline $\mathrm{C}(23)-\mathrm{N}(3)-\mathrm{C}(22)-\mathrm{N}(4)$ & $-0.7(2)$ \\
\hline $\mathrm{C}(25)-\mathrm{N}(3)-\mathrm{C}(22)-\mathrm{N}(4)$ & $178.49(17)$ \\
\hline $\mathrm{C}(23)-\mathrm{N}(3)-\mathrm{C}(22)-\mathrm{Pd}$ & $159.81(14)$ \\
\hline $\mathrm{C}(25)-\mathrm{N}(3)-\mathrm{C}(22)-\mathrm{Pd}$ & $-21.0(3)$ \\
\hline $\mathrm{O}(1)-\mathrm{Pd}-\mathrm{C}(22)-\mathrm{N}(4)$ & $71.63(17)$ \\
\hline $\mathrm{O}(2)-\mathrm{Pd}-\mathrm{C}(22)-\mathrm{N}(4)$ & $72.67(14)$ \\
\hline $\mathrm{C}(1)-\mathrm{Pd}-\mathrm{C}(22)-\mathrm{N}(4)$ & $-109.09(13)$ \\
\hline $\mathrm{O}(1)-\mathrm{Pd}-\mathrm{C}(22)-\mathrm{N}(3)$ & $-85.47(17)$ \\
\hline $\mathrm{O}(2)-\mathrm{Pd}-\mathrm{C}(22)-\mathrm{N}(3)$ & $-84.43(15)$ \\
\hline $\mathrm{C}(1)-\mathrm{Pd}-\mathrm{C}(22)-\mathrm{N}(3)$ & $93.81(16)$ \\
\hline $\mathrm{C}(22)-\mathrm{N}(3)-\mathrm{C}(23)-\mathrm{C}(24)$ & $0.6(3)$ \\
\hline $\mathrm{C}(25)-\mathrm{N}(3)-\mathrm{C}(23)-\mathrm{C}(24)$ & $-178.73(18)$ \\
\hline $\mathrm{N}(3)-\mathrm{C}(23)-\mathrm{C}(24)-\mathrm{N}(4)$ & $-0.2(3)$ \\
\hline $\mathrm{C}(22)-\mathrm{N}(4)-\mathrm{C}(24)-\mathrm{C}(23)$ & $-0.3(2)$ \\
\hline $\mathrm{C}(34)-\mathrm{N}(4)-\mathrm{C}(24)-\mathrm{C}(23)$ & $179.21(18)$ \\
\hline $\mathrm{C}(22)-\mathrm{N}(3)-\mathrm{C}(25)-\mathrm{C}(30)$ & $-111.9(2)$ \\
\hline $\mathrm{C}(23)-\mathrm{N}(3)-\mathrm{C}(25)-\mathrm{C}(30)$ & $67.2(2)$ \\
\hline $\mathrm{C}(22)-\mathrm{N}(3)-\mathrm{C}(25)-\mathrm{C}(26)$ & $77.6(2)$ \\
\hline $\mathrm{C}(23)-\mathrm{N}(3)-\mathrm{C}(25)-\mathrm{C}(26)$ & $-103.2(2)$ \\
\hline $\mathrm{C}(30)-\mathrm{C}(25)-\mathrm{C}(26)-\mathrm{C}(27)$ & $-0.1(3)$ \\
\hline $\mathrm{N}(3)-\mathrm{C}(25)-\mathrm{C}(26)-\mathrm{C}(27)$ & $169.90(15)$ \\
\hline $\mathrm{C}(30)-\mathrm{C}(25)-\mathrm{C}(26)-\mathrm{C}(31)$ & $-178.79(17)$ \\
\hline $\mathrm{N}(3)-\mathrm{C}(25)-\mathrm{C}(26)-\mathrm{C}(31)$ & $-8.8(2)$ \\
\hline $\mathrm{C}(25)-\mathrm{C}(26)-\mathrm{C}(27)-\mathrm{C}(28)$ & $-0.4(3)$ \\
\hline $\mathrm{C}(31)-\mathrm{C}(26)-\mathrm{C}(27)-\mathrm{C}(28)$ & $178.26(17)$ \\
\hline $\mathrm{C}(26)-\mathrm{C}(27)-\mathrm{C}(28)-\mathrm{C}(29)$ & $0.8(3)$ \\
\hline $\mathrm{C}(26)-\mathrm{C}(27)-\mathrm{C}(28)-\mathrm{C}(32)$ & $179.75(17)$ \\
\hline $\mathrm{C}(27)-\mathrm{C}(28)-\mathrm{C}(29)-\mathrm{C}(30)$ & $-0.7(3)$ \\
\hline $\mathrm{C}(32)-\mathrm{C}(28)-\mathrm{C}(29)-\mathrm{C}(30)$ & $-179.66(17)$ \\
\hline $\mathrm{C}(26)-\mathrm{C}(25)-\mathrm{C}(30)-\mathrm{C}(29)$ & $0.2(3)$ \\
\hline $\mathrm{N}(3)-\mathrm{C}(25)-\mathrm{C}(30)-\mathrm{C}(29)$ & $-169.97(16)$ \\
\hline$C(26)-C(25)-C(30)-C(33)$ & $179.25(18)$ \\
\hline $\mathrm{N}(3)-\mathrm{C}(25)-\mathrm{C}(30)-\mathrm{C}(33)$ & $9.1(3)$ \\
\hline $\mathrm{C}(28)-\mathrm{C}(29)-\mathrm{C}(30)-\mathrm{C}(25)$ & $0.2(3)$ \\
\hline $\mathrm{C}(28)-\mathrm{C}(29)-\mathrm{C}(30)-\mathrm{C}(33)$ & $-178.84(18)$ \\
\hline $\mathrm{C}(22)-\mathrm{N}(4)-\mathrm{C}(34)-\mathrm{C}(35)$ & $-97.56(19)$ \\
\hline $\mathrm{C}(24)-\mathrm{N}(4)-\mathrm{C}(34)-\mathrm{C}(35)$ & $83.0(2)$ \\
\hline $\mathrm{C}(22)-\mathrm{N}(4)-\mathrm{C}(34)-\mathrm{C}(39)$ & $88.9(2)$ \\
\hline $\mathrm{C}(24)-\mathrm{N}(4)-\mathrm{C}(34)-\mathrm{C}(39)$ & $-90.6(2)$ \\
\hline$C(39)-C(34)-C(35)-C(36)$ & $-2.4(2)$ \\
\hline $\mathrm{N}(4)-\mathrm{C}(34)-\mathrm{C}(35)-\mathrm{C}(36)$ & $-175.67(14)$ \\
\hline $\mathrm{C}(39)-\mathrm{C}(34)-\mathrm{C}(35)-\mathrm{C}(40)$ & $176.84(16)$ \\
\hline $\mathrm{N}(4)-\mathrm{C}(34)-\mathrm{C}(35)-\mathrm{C}(40)$ & $3.6(2)$ \\
\hline$C(34)-C(35)-C(36)-C(37)$ & $-1.0(2)$ \\
\hline $\mathrm{C}(40)-\mathrm{C}(35)-\mathrm{C}(36)-\mathrm{C}(37)$ & $179.73(16)$ \\
\hline $\mathrm{C}(35)-\mathrm{C}(36)-\mathrm{C}(37)-\mathrm{C}(38)$ & $2.4(2)$ \\
\hline $\mathrm{C}(35)-\mathrm{C}(36)-\mathrm{C}(37)-\mathrm{C}(41)$ & $-177.33(15)$ \\
\hline
\end{tabular}


$\mathrm{C}(36)-\mathrm{C}(37)-\mathrm{C}(38)-\mathrm{C}(39)$

$\mathrm{C}(41)-\mathrm{C}(37)-\mathrm{C}(38)-\mathrm{C}(39)$

$\mathrm{C}(37)-\mathrm{C}(38)-\mathrm{C}(39)-\mathrm{C}(34)$

$\mathrm{C}(37)-\mathrm{C}(38)-\mathrm{C}(39)-\mathrm{C}(42)$

$\mathrm{C}(35)-\mathrm{C}(34)-\mathrm{C}(39)-\mathrm{C}(38)$

$\mathrm{N}(4)-\mathrm{C}(34)-\mathrm{C}(39)-\mathrm{C}(38)$

$\mathrm{C}(35)-\mathrm{C}(34)-\mathrm{C}(39)-\mathrm{C}(42)$

$\mathrm{N}(4)-\mathrm{C}(34)-\mathrm{C}(39)-\mathrm{C}(42)$
$-0.4(2)$

$179.32(15)$

$-2.8(2)$

$174.53(15)$

$4.3(2)$

177.61(13)

$-173.10(15)$

$0.2(2)$

Symmetry transformations used to generate equivalent atoms: 


\section{X-ray crystallographic Analysis of $\mathrm{Pd}(\mathrm{IMes})_{2}(\mathrm{OAc})_{2},(5)$.}

A colorless crystal with approximate dimensions $0.43 \times 0.35 \times 0.22 \mathrm{~mm}^{3}$ was selected under oil under ambient conditions and attached to the tip of a glass capillary. The crystal was mounted in a stream of cold nitrogen at 100(2) K and centered in the X-ray beam by using a video camera.

The crystal evaluation and data collection were performed on a Bruker CCD-1000 diffractometer with $\mathrm{Mo} \mathrm{K}_{\alpha}(\lambda=0.71073 \AA)$ radiation and the diffractometer to crystal distance of $4.8 \mathrm{~cm}$.

The initial cell constants were obtained from three series of $\omega$ scans at different starting angles. Each series consisted of 30 frames collected at intervals of $0.3^{\circ}$ in a $6^{\circ}$ range about $\omega$ with the exposure time of 8 seconds per frame. A total of 264 reflections was obtained. The reflections were successfully indexed by an automated indexing routine built in the SMART program. The final cell constants were calculated from a set of 30436 strong reflections from the actual data collection.

The data were collected by using the hemisphere data collection routine. The reciprocal space was surveyed to the extent of a full sphere to a resolution of $0.80 \AA$. A total of 48596 data were harvested by collecting eight sets of frames with $0.3^{\circ}$ scans in $\omega$ with an exposure time 10 sec per frame. These highly redundant datasets were corrected for Lorentz and polarization effects. The absorption correction was based on fitting a function to the empirical transmission surface as sampled by multiple equivalent measurements ${ }^{3}$.

\section{Structure Solution and Refinement}

The systematic absences in the diffraction data were uniquely consistent for the space group $P 2{ }_{1} / n$ that yielded chemically reasonable and computationally stable results of refinement ${ }^{3}$.

A successful solution by the direct methods provided most non-hydrogen atoms from the $E$-map. The remaining non-hydrogen atoms were located in an alternating series of least-squares cycles and difference Fourier maps. All non-hydrogen atoms were refined with anisotropic displacement coefficients. All hydrogen atoms were included in the structure factor calculation 
at idealized positions and were allowed to ride on the neighboring atoms with relative isotropic displacement coefficients. The complex occupies a crystallographic inversion center.

The final least-squares refinement of 257 parameters against 5094 data resulted in residuals $R$ (based on $F^{2}$ for $I \geq 2 \sigma$ ) and $w R$ (based on $F^{2}$ for all data) of 0.0323 and 0.0868 , respectively. The final difference Fourier map was featureless.

The ORTEP diagram is drawn with $50 \%$ probability ellipsoids. 


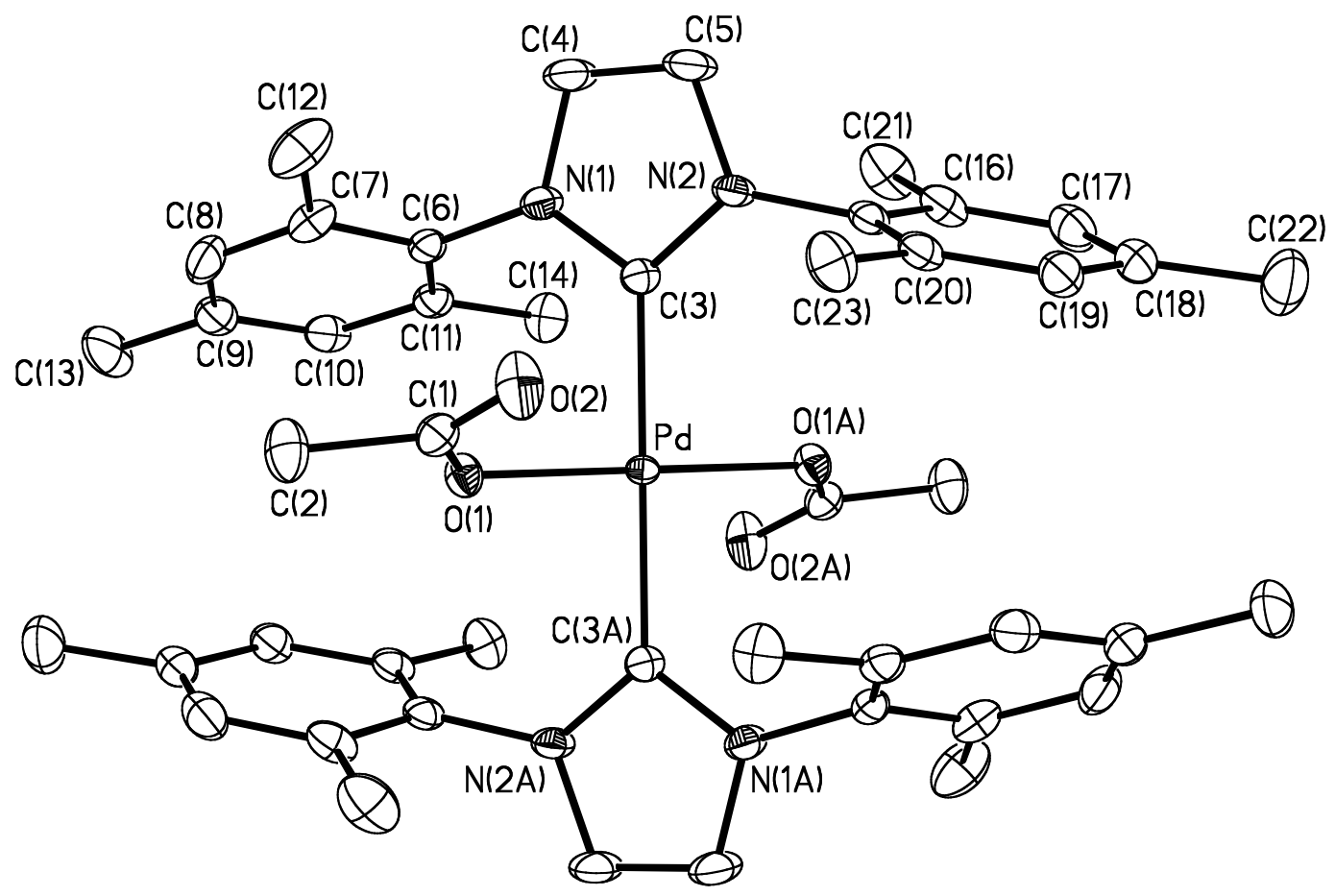

Figure S17. A molecular drawing of (5) with 50\% probability ellipsoids. 
Table S14. Crystal data and structure refinement for (5).

\begin{tabular}{|c|c|c|}
\hline Identification code & \multicolumn{2}{|l|}{ sta15 } \\
\hline Empirical formula & \multicolumn{2}{|l|}{$\mathrm{C}_{46} \mathrm{H}_{54} \mathrm{~N}_{4} \mathrm{O}_{4} \mathrm{Pd}$} \\
\hline Formula weight & \multicolumn{2}{|l|}{833.33} \\
\hline Temperature & \multicolumn{2}{|l|}{$100(2) \mathrm{K}$} \\
\hline Wavelength & \multicolumn{2}{|l|}{$0.71073 \AA$} \\
\hline Crystal system & \multicolumn{2}{|l|}{ Monoclinic } \\
\hline Space group & \multicolumn{2}{|l|}{$\mathrm{P} 2_{1} / \mathrm{n}$} \\
\hline \multirow[t]{3}{*}{ Unit cell dimensions } & $\mathrm{a}=13.0229(13) \AA$ & $\alpha=90^{\circ}$ \\
\hline & $\mathrm{b}=9.9968(10) \AA$ & $\beta=106.1275(14)^{\circ}$. \\
\hline & $c=16.3609(16) \AA$ & $\gamma=90^{\circ}$ \\
\hline Volume & \multicolumn{2}{|l|}{$2046.2(4) \AA^{3}$} \\
\hline Z & \multicolumn{2}{|l|}{2} \\
\hline Density (calculated) & \multicolumn{2}{|l|}{$1.353 \mathrm{Mg} / \mathrm{m}^{3}$} \\
\hline Absorption coefficient & \multicolumn{2}{|l|}{$0.502 \mathrm{~mm}^{-1}$} \\
\hline $\mathrm{F}(000)$ & \multicolumn{2}{|l|}{872} \\
\hline Crystal size & \multicolumn{2}{|c|}{$0.43 \times 0.35 \times 0.22 \mathrm{~mm}^{3}$} \\
\hline Theta range for data collection & \multicolumn{2}{|l|}{2.35 to $28.34^{\circ}$} \\
\hline Index ranges & \multicolumn{2}{|c|}{$-17<=\mathrm{h}<=17,-13<=\mathrm{k}<=13,-21<=1<=21$} \\
\hline Reflections collected & \multicolumn{2}{|l|}{48596} \\
\hline Independent reflections & \multicolumn{2}{|c|}{$5094[\mathrm{R}(\mathrm{int})=0.0427]$} \\
\hline Completeness to theta $=28.34^{\circ}$ & \multicolumn{2}{|l|}{$99.6 \%$} \\
\hline Absorption correction & \multicolumn{2}{|c|}{ Multiscan with SADABS } \\
\hline Max. and min. transmission & \multicolumn{2}{|l|}{0.8977 and 0.8132} \\
\hline Refinement method & \multicolumn{2}{|c|}{ Full-matrix least-squares on $\mathrm{F}^{2}$} \\
\hline Data / restraints / parameters & \multicolumn{2}{|l|}{5094 / 0 / 257} \\
\hline Goodness-of-fit on $\mathrm{F}^{2}$ & \multicolumn{2}{|l|}{1.016} \\
\hline Final $R$ indices $[\mathrm{I}>2 \operatorname{sigma}(\mathrm{I})]$ & \multicolumn{2}{|c|}{$\mathrm{R} 1=0.0323, \mathrm{wR} 2=0.0851$} \\
\hline $\mathrm{R}$ indices (all data) & \multicolumn{2}{|c|}{$\mathrm{R} 1=0.0343, \mathrm{wR} 2=0.0868$} \\
\hline Largest diff. peak and hole & \multicolumn{2}{|c|}{1.030 and -0.317 e. $\AA^{-3}$} \\
\hline
\end{tabular}


Table S15. Atomic coordinates $\left(\mathrm{x} 10^{4}\right)$ and equivalent isotropic displacement parameters $\left(\AA^{2} \times 10^{3}\right)$ for (5). $U(e q)$ is defined as one third of the trace of the orthogonalized $U^{i j}$ tensor.

\begin{tabular}{|c|c|c|c|c|}
\hline & $\mathrm{x}$ & $\mathrm{y}$ & $\mathrm{z}$ & $\mathrm{U}(\mathrm{eq})$ \\
\hline$\overline{\mathrm{Pd}}$ & 0 & 0 & 0 & $11(1)$ \\
\hline $\mathrm{O}(1)$ & $-466(1)$ & $-1536(1)$ & $607(1)$ & $18(1)$ \\
\hline $\mathrm{O}(2)$ & 1197(1) & $-2262(2)$ & $1214(1)$ & $30(1)$ \\
\hline $\mathrm{N}(1)$ & 277(1) & $1039(2)$ & 1811(1) & $20(1)$ \\
\hline $\mathrm{N}(2)$ & $1652(1)$ & 1557(2) & $1396(1)$ & $20(1)$ \\
\hline $\mathrm{C}(1)$ & $223(1)$ & $-2333(2)$ & 1071(1) & $19(1)$ \\
\hline$C(2)$ & $-271(2)$ & $-3466(2)$ & $1454(1)$ & $28(1)$ \\
\hline$C(3)$ & $675(1)$ & $957(2)$ & $1124(1)$ & $16(1)$ \\
\hline$C(4)$ & 1001(2) & 1657(2) & 2496(1) & $30(1)$ \\
\hline$C(5)$ & 1863(2) & 1972(2) & $2240(1)$ & $29(1)$ \\
\hline$C(6)$ & $-775(1)$ & $644(2)$ & $1842(1)$ & $18(1)$ \\
\hline$C(7)$ & $-869(2)$ & $-380(2)$ & 2398(1) & $25(1)$ \\
\hline$C(8)$ & $-1889(2)$ & $-718(2)$ & $2440(1)$ & $29(1)$ \\
\hline $\mathrm{C}(9)$ & $-2794(2)$ & $-59(2)$ & 1959(1) & $27(1)$ \\
\hline$C(10)$ & $-2666(1)$ & $971(2)$ & $1426(1)$ & $23(1)$ \\
\hline$C(11)$ & $-1662(1)$ & 1353(2) & $1357(1)$ & $20(1)$ \\
\hline$C(12)$ & $98(2)$ & $-1087(2)$ & 2952(1) & $37(1)$ \\
\hline$C(13)$ & $-3888(2)$ & $-436(3)$ & $2024(2)$ & $43(1)$ \\
\hline$C(14)$ & $-1557(2)$ & 2512(2) & 799(1) & $27(1)$ \\
\hline$C(15)$ & $2337(1)$ & 1941(2) & $877(1)$ & 19(1) \\
\hline$C(16)$ & $2268(1)$ & $3259(2)$ & $585(1)$ & $24(1)$ \\
\hline$C(17)$ & $2942(2)$ & $3653(2)$ & $104(1)$ & $28(1)$ \\
\hline$C(18)$ & $3681(2)$ & 2777(2) & $-77(1)$ & $28(1)$ \\
\hline C(19) & $3726(1)$ & 1481(2) & $228(1)$ & $24(1)$ \\
\hline$C(20)$ & $3064(1)$ & 1031(2) & $715(1)$ & $20(1)$ \\
\hline$C(21)$ & $1482(2)$ & $4235(2)$ & $783(2)$ & $35(1)$ \\
\hline$C(22)$ & $4422(2)$ & $3257(3)$ & $-583(2)$ & $47(1)$ \\
\hline$C(23)$ & $3149(2)$ & $-367(2)$ & $1060(1)$ & $27(1)$ \\
\hline
\end{tabular}


Table S16. Bond lengths $[\AA]$ and angles $\left[^{\circ}\right]$ for (5).

\begin{tabular}{|c|c|c|c|}
\hline$\overline{\mathrm{Pd}-\mathrm{O}(1)}$ & $2.0120(11)$ & $\bar{C}(7)-C(8)$ & $1.390(3)$ \\
\hline $\mathrm{Pd}-\mathrm{O}(1) \# 1$ & $2.0120(11)$ & $C(7)-C(12)$ & $1.507(3)$ \\
\hline $\mathrm{Pd}-\mathrm{C}(3)$ & $2.0427(16)$ & $\mathrm{C}(8)-\mathrm{C}(9)$ & $1.389(3)$ \\
\hline $\mathrm{Pd}-\mathrm{C}(3) \# 1$ & $2.0427(16)$ & $\mathrm{C}(9)-\mathrm{C}(10)$ & $1.389(3)$ \\
\hline $\mathrm{O}(1)-\mathrm{C}(1)$ & $1.280(2)$ & $\mathrm{C}(9)-\mathrm{C}(13)$ & $1.506(3)$ \\
\hline $\mathrm{O}(2)-\mathrm{C}(1)$ & $1.226(2)$ & $\mathrm{C}(10)-\mathrm{C}(11)$ & $1.397(2)$ \\
\hline $\mathrm{N}(1)-\mathrm{C}(3)$ & $1.363(2)$ & $\mathrm{C}(11)-\mathrm{C}(14)$ & $1.504(2)$ \\
\hline $\mathrm{N}(1)-\mathrm{C}(4)$ & $1.393(2)$ & $C(15)-C(20)$ & $1.391(2)$ \\
\hline $\mathrm{N}(1)-\mathrm{C}(6)$ & $1.440(2)$ & $C(15)-C(16)$ & $1.396(3)$ \\
\hline $\mathrm{N}(2)-\mathrm{C}(3)$ & $1.365(2)$ & $C(16)-C(17)$ & $1.389(3)$ \\
\hline $\mathrm{N}(2)-\mathrm{C}(5)$ & $1.393(2)$ & $C(16)-C(21)$ & $1.512(3)$ \\
\hline $\mathrm{N}(2)-\mathrm{C}(15)$ & $1.444(2)$ & $\mathrm{C}(17)-\mathrm{C}(18)$ & $1.392(3)$ \\
\hline $\mathrm{C}(1)-\mathrm{C}(2)$ & $1.522(2)$ & $C(18)-C(19)$ & $1.384(3)$ \\
\hline$C(4)-C(5)$ & $1.339(3)$ & $C(18)-C(22)$ & $1.514(3)$ \\
\hline $\mathrm{C}(6)-\mathrm{C}(7)$ & $1.398(3)$ & $C(19)-C(20)$ & $1.401(2)$ \\
\hline$C(6)-C(11)$ & $1.399(2)$ & $C(20)-C(23)$ & $1.499(3)$ \\
\hline $\mathrm{O}(1)-\mathrm{Pd}-\mathrm{O}(1) \# 1$ & $180.00(7)$ & $\mathrm{C}(8)-\mathrm{C}(7)-\mathrm{C}(12)$ & $120.40(19)$ \\
\hline $\mathrm{O}(1)-\mathrm{Pd}-\mathrm{C}(3)$ & $91.49(6)$ & $C(6)-C(7)-C(12)$ & $121.69(18)$ \\
\hline $\mathrm{O}(1) \# 1-\mathrm{Pd}-\mathrm{C}(3)$ & $88.51(6)$ & $\mathrm{C}(9)-\mathrm{C}(8)-\mathrm{C}(7)$ & $121.95(18)$ \\
\hline O(1)-Pd-C(3)\#1 & $88.51(6)$ & $\mathrm{C}(8)-\mathrm{C}(9)-\mathrm{C}(10)$ & $118.47(18)$ \\
\hline $\mathrm{O}(1) \# 1-\mathrm{Pd}-\mathrm{C}(3) \# 1$ & $91.49(6)$ & $\mathrm{C}(8)-\mathrm{C}(9)-\mathrm{C}(13)$ & $120.8(2)$ \\
\hline $\mathrm{C}(3)-\mathrm{Pd}-\mathrm{C}(3) \# 1$ & $180.00(6)$ & $\mathrm{C}(10)-\mathrm{C}(9)-\mathrm{C}(13)$ & $120.7(2)$ \\
\hline C(1)-O(1)-Pd & $120.64(11)$ & $\mathrm{C}(9)-\mathrm{C}(10)-\mathrm{C}(11)$ & $122.08(18)$ \\
\hline $\mathrm{C}(3)-\mathrm{N}(1)-\mathrm{C}(4)$ & $111.21(14)$ & $\mathrm{C}(10)-\mathrm{C}(11)-\mathrm{C}(6)$ & $117.44(16)$ \\
\hline $\mathrm{C}(3)-\mathrm{N}(1)-\mathrm{C}(6)$ & $126.55(13)$ & $\mathrm{C}(10)-\mathrm{C}(11)-\mathrm{C}(14)$ & $120.28(16)$ \\
\hline $\mathrm{C}(4)-\mathrm{N}(1)-\mathrm{C}(6)$ & $122.10(14)$ & $\mathrm{C}(6)-\mathrm{C}(11)-\mathrm{C}(14)$ & $122.26(16)$ \\
\hline $\mathrm{C}(3)-\mathrm{N}(2)-\mathrm{C}(5)$ & $111.29(14)$ & $C(20)-C(15)-C(16)$ & $122.36(16)$ \\
\hline $\mathrm{C}(3)-\mathrm{N}(2)-\mathrm{C}(15)$ & $126.70(13)$ & $\mathrm{C}(20)-\mathrm{C}(15)-\mathrm{N}(2)$ & $120.19(16)$ \\
\hline $\mathrm{C}(5)-\mathrm{N}(2)-\mathrm{C}(15)$ & $121.28(14)$ & $\mathrm{C}(16)-\mathrm{C}(15)-\mathrm{N}(2)$ & $117.40(15)$ \\
\hline $\mathrm{O}(2)-\mathrm{C}(1)-\mathrm{O}(1)$ & $126.54(16)$ & $\mathrm{C}(17)-\mathrm{C}(16)-\mathrm{C}(15)$ & $118.04(17)$ \\
\hline $\mathrm{O}(2)-\mathrm{C}(1)-\mathrm{C}(2)$ & $119.84(15)$ & $\mathrm{C}(17)-\mathrm{C}(16)-\mathrm{C}(21)$ & $120.71(18)$ \\
\hline $\mathrm{O}(1)-\mathrm{C}(1)-\mathrm{C}(2)$ & 113.61(14) & $C(15)-C(16)-C(21)$ & $121.24(17)$ \\
\hline $\mathrm{N}(1)-\mathrm{C}(3)-\mathrm{N}(2)$ & $103.78(13)$ & $\mathrm{C}(16)-\mathrm{C}(17)-\mathrm{C}(18)$ & $121.67(18)$ \\
\hline $\mathrm{N}(1)-\mathrm{C}(3)-\mathrm{Pd}$ & $127.09(12)$ & $\mathrm{C}(19)-\mathrm{C}(18)-\mathrm{C}(17)$ & $118.42(17)$ \\
\hline $\mathrm{N}(2)-\mathrm{C}(3)-\mathrm{Pd}$ & $128.92(12)$ & $\mathrm{C}(19)-\mathrm{C}(18)-\mathrm{C}(22)$ & $121.57(19)$ \\
\hline $\mathrm{C}(5)-\mathrm{C}(4)-\mathrm{N}(1)$ & $106.97(15)$ & $\mathrm{C}(17)-\mathrm{C}(18)-\mathrm{C}(22)$ & $120.00(19)$ \\
\hline $\mathrm{C}(4)-\mathrm{C}(5)-\mathrm{N}(2)$ & $106.72(15)$ & $C(18)-C(19)-C(20)$ & $122.27(17)$ \\
\hline$C(7)-C(6)-C(11)$ & $122.13(16)$ & $\mathrm{C}(15)-\mathrm{C}(20)-\mathrm{C}(19)$ & $117.22(17)$ \\
\hline $\mathrm{C}(7)-\mathrm{C}(6)-\mathrm{N}(1)$ & $118.71(15)$ & $\mathrm{C}(15)-\mathrm{C}(20)-\mathrm{C}(23)$ & $121.38(16)$ \\
\hline $\mathrm{C}(11)-\mathrm{C}(6)-\mathrm{N}(1)$ & $119.02(15)$ & $\mathrm{C}(19)-\mathrm{C}(20)-\mathrm{C}(23)$ & $121.39(16)$ \\
\hline$C(8)-C(7)-C(6)$ & $117.89(17)$ & & \\
\hline
\end{tabular}

Symmetry transformations used to generate equivalent atoms: $\# 1-\mathrm{x},-\mathrm{y},-\mathrm{z}$ 
Table S17. Anisotropic displacement parameters $\left(\AA^{2} \times 10^{3}\right)$ for (5). The anisotropic displacement factor exponent takes the form: $-2 \pi^{2}\left[h^{2} a^{* 2} U^{11}+\ldots+2 h k a^{*} b^{*} U^{12}\right]$

\begin{tabular}{lcccccc}
\hline & $\mathrm{U}^{11}$ & $\mathrm{U}^{22}$ & $\mathrm{U}^{33}$ & $\mathrm{U}^{23}$ & $\mathrm{U}^{13}$ & $\mathrm{U}^{12}$ \\
\hline $\mathrm{Pd}$ & $11(1)$ & $12(1)$ & $11(1)$ & $0(1)$ & $1(1)$ & $1(1)$ \\
$\mathrm{O}(1)$ & $16(1)$ & $17(1)$ & $19(1)$ & $4(1)$ & $3(1)$ & $0(1)$ \\
$\mathrm{O}(2)$ & $18(1)$ & $33(1)$ & $36(1)$ & $17(1)$ & $4(1)$ & $3(1)$ \\
$\mathrm{N}(1)$ & $15(1)$ & $29(1)$ & $14(1)$ & $-6(1)$ & $3(1)$ & $-2(1)$ \\
$\mathrm{N}(2)$ & $14(1)$ & $26(1)$ & $18(1)$ & $-8(1)$ & $3(1)$ & $-2(1)$ \\
$\mathrm{C}(1)$ & $20(1)$ & $20(1)$ & $17(1)$ & $2(1)$ & $5(1)$ & $1(1)$ \\
$\mathrm{C}(2)$ & $26(1)$ & $25(1)$ & $34(1)$ & $13(1)$ & $10(1)$ & $2(1)$ \\
$\mathrm{C}(3)$ & $13(1)$ & $18(1)$ & $16(1)$ & $-1(1)$ & $3(1)$ & $2(1)$ \\
$\mathrm{C}(4)$ & $23(1)$ & $48(1)$ & $18(1)$ & $-14(1)$ & $4(1)$ & $-5(1)$ \\
$\mathrm{C}(5)$ & $20(1)$ & $43(1)$ & $21(1)$ & $-15(1)$ & $3(1)$ & $-7(1)$ \\
$\mathrm{C}(6)$ & $16(1)$ & $24(1)$ & $15(1)$ & $-4(1)$ & $6(1)$ & $-2(1)$ \\
$\mathrm{C}(7)$ & $29(1)$ & $29(1)$ & $17(1)$ & $-1(1)$ & $8(1)$ & $4(1)$ \\
$\mathrm{C}(8)$ & $39(1)$ & $29(1)$ & $24(1)$ & $0(1)$ & $18(1)$ & $-4(1)$ \\
$\mathrm{C}(9)$ & $26(1)$ & $33(1)$ & $26(1)$ & $-13(1)$ & $15(1)$ & $-9(1)$ \\
$\mathrm{C}(10)$ & $18(1)$ & $30(1)$ & $23(1)$ & $-7(1)$ & $6(1)$ & $1(1)$ \\
$\mathrm{C}(11)$ & $21(1)$ & $20(1)$ & $19(1)$ & $-4(1)$ & $6(1)$ & $0(1)$ \\
$\mathrm{C}(12)$ & $43(1)$ & $47(1)$ & $21(1)$ & $10(1)$ & $8(1)$ & $16(1)$ \\
$\mathrm{C}(13)$ & $34(1)$ & $54(1)$ & $51(1)$ & $-23(1)$ & $28(1)$ & $-21(1)$ \\
$\mathrm{C}(14)$ & $28(1)$ & $25(1)$ & $31(1)$ & $5(1)$ & $10(1)$ & $4(1)$ \\
$\mathrm{C}(15)$ & $13(1)$ & $23(1)$ & $19(1)$ & $-5(1)$ & $2(1)$ & $-3(1)$ \\
$\mathrm{C}(16)$ & $18(1)$ & $22(1)$ & $30(1)$ & $-6(1)$ & $3(1)$ & $-2(1)$ \\
$\mathrm{C}(17)$ & $27(1)$ & $23(1)$ & $31(1)$ & $0(1)$ & $4(1)$ & $-6(1)$ \\
$\mathrm{C}(18)$ & $29(1)$ & $30(1)$ & $26(1)$ & $-8(1)$ & $11(1)$ & $-11(1)$ \\
$\mathrm{C}(19)$ & $21(1)$ & $26(1)$ & $27(1)$ & $-8(1)$ & $10(1)$ & $-3(1)$ \\
$\mathrm{C}(20)$ & $14(1)$ & $23(1)$ & $22(1)$ & $-6(1)$ & $3(1)$ & $-3(1)$ \\
$\mathrm{C}(21)$ & $28(1)$ & $22(1)$ & $54(1)$ & $-6(1)$ & $12(1)$ & $3(1)$ \\
$\mathrm{C}(22)$ & $65(2)$ & $38(1)$ & $53(1)$ & $-12(1)$ & $41(1)$ & $-20(1)$ \\
$\mathrm{C}(23)$ & $18(1)$ & $25(1)$ & $38(1)$ & $2(1)$ & $8(1)$ & $2(1)$ \\
& & & & & & \\
\hline
\end{tabular}


Table S18. Hydrogen coordinates ( $\left.\times 10^{4}\right)$ and isotropic displacement parameters $\left(\AA^{2} \times 10^{3}\right)$ for (5).

\begin{tabular}{|c|c|c|c|c|}
\hline & $\mathrm{x}$ & $y$ & $\mathrm{z}$ & $\mathrm{U}(\mathrm{eq})$ \\
\hline $\mathrm{H}(2 \mathrm{~A})$ & -451 & -4209 & 1048 & 42 \\
\hline $\mathrm{H}(2 \mathrm{~B})$ & -922 & -3146 & 1581 & 42 \\
\hline $\mathrm{H}(2 \mathrm{C})$ & 240 & -3774 & 1980 & 42 \\
\hline $\mathrm{H}(4)$ & 903 & 1823 & 3041 & 36 \\
\hline $\mathrm{H}(5)$ & 2495 & 2397 & 2569 & 35 \\
\hline $\mathrm{H}(8)$ & -1968 & -1421 & 2808 & 34 \\
\hline $\mathrm{H}(10)$ & -3282 & 1430 & 1097 & 28 \\
\hline $\mathrm{H}(12 \mathrm{~A})$ & 622 & -1225 & 2631 & 56 \\
\hline $\mathrm{H}(12 \mathrm{~B})$ & -117 & -1954 & 3130 & 56 \\
\hline $\mathrm{H}(12 \mathrm{C})$ & 417 & -540 & 3456 & 56 \\
\hline $\mathrm{H}(13 \mathrm{~A})$ & -3952 & -1413 & 2027 & 65 \\
\hline $\mathrm{H}(13 \mathrm{~B})$ & -4432 & -69 & 1537 & 65 \\
\hline $\mathrm{H}(13 \mathrm{C})$ & -3990 & -72 & 2552 & 65 \\
\hline $\mathrm{H}(14 \mathrm{~A})$ & -1436 & 2174 & 272 & 41 \\
\hline $\mathrm{H}(14 \mathrm{~B})$ & -951 & 3072 & 1098 & 41 \\
\hline $\mathrm{H}(14 \mathrm{C})$ & -2214 & 3044 & 664 & 41 \\
\hline $\mathrm{H}(17)$ & 2897 & 4542 & -108 & 33 \\
\hline $\mathrm{H}(19)$ & 4223 & 875 & 102 & 29 \\
\hline $\mathrm{H}(21 \mathrm{~A})$ & 1494 & 5073 & 476 & 52 \\
\hline $\mathrm{H}(21 \mathrm{~B})$ & 1682 & 4412 & 1396 & 52 \\
\hline $\mathrm{H}(21 \mathrm{C})$ & 762 & 3851 & 606 & 52 \\
\hline $\mathrm{H}(22 \mathrm{~A})$ & 4981 & 2587 & -552 & 71 \\
\hline $\mathrm{H}(22 \mathrm{~B})$ & 4752 & 4105 & -345 & 71 \\
\hline $\mathrm{H}(22 \mathrm{C})$ & 4014 & 3390 & -1177 & 71 \\
\hline $\mathrm{H}(23 \mathrm{~A})$ & 3474 & -346 & 1677 & 40 \\
\hline $\mathrm{H}(23 \mathrm{~B})$ & 3595 & -905 & 791 & 40 \\
\hline $\mathrm{H}(23 \mathrm{C})$ & 2434 & -763 & 937 & 40 \\
\hline
\end{tabular}


Table S19. Torsion angles $\left[{ }^{\circ}\right]$ for (5).

\begin{tabular}{|c|c|c|c|}
\hline$\overline{\mathrm{O}(1) \# 1-\mathrm{Pd}-\mathrm{O}(1)-\mathrm{C}(1)}$ & $173(58)$ & $\mathrm{C}(17)-\mathrm{C}(18)-\mathrm{C}(19)-\mathrm{C}(20)$ & $-0.7(3)$ \\
\hline $\mathrm{C}(3)-\mathrm{Pd}-\mathrm{O}(1)-\mathrm{C}(1)$ & $-68.84(13)$ & $\mathrm{C}(22)-\mathrm{C}(18)-\mathrm{C}(19)-\mathrm{C}(20)$ & $178.37(19)$ \\
\hline C(3)\#1-Pd-O(1)-C(1) & 111.16(13) & $\mathrm{C}(16)-\mathrm{C}(15)-\mathrm{C}(20)-\mathrm{C}(19)$ & $-1.0(2)$ \\
\hline $\mathrm{Pd}-\mathrm{O}(1)-\mathrm{C}(1)-\mathrm{O}(2)$ & $1.5(2)$ & $\mathrm{N}(2)-\mathrm{C}(15)-\mathrm{C}(20)-\mathrm{C}(19)$ & $-178.15(15)$ \\
\hline $\mathrm{Pd}-\mathrm{O}(1)-\mathrm{C}(1)-\mathrm{C}(2)$ & $-177.78(12)$ & $\mathrm{C}(16)-\mathrm{C}(15)-\mathrm{C}(20)-\mathrm{C}(23)$ & $177.93(16)$ \\
\hline $\mathrm{C}(4)-\mathrm{N}(1)-\mathrm{C}(3)-\mathrm{N}(2)$ & $-1.0(2)$ & $\mathrm{N}(2)-\mathrm{C}(15)-\mathrm{C}(20)-\mathrm{C}(23)$ & $0.8(2)$ \\
\hline $\mathrm{C}(6)-\mathrm{N}(1)-\mathrm{C}(3)-\mathrm{N}(2)$ & $174.82(16)$ & $\mathrm{C}(18)-\mathrm{C}(19)-\mathrm{C}(20)-\mathrm{C}(15)$ & $0.8(3)$ \\
\hline $\mathrm{C}(4)-\mathrm{N}(1)-\mathrm{C}(3)-\mathrm{Pd}$ & $174.08(14)$ & $\mathrm{C}(18)-\mathrm{C}(19)-\mathrm{C}(20)-\mathrm{C}(23)$ & $-178.15(17)$ \\
\hline $\mathrm{C}(6)-\mathrm{N}(1)-\mathrm{C}(3)-\mathrm{Pd}$ & $-10.1(2)$ & & \\
\hline $\mathrm{C}(5)-\mathrm{N}(2)-\mathrm{C}(3)-\mathrm{N}(1)$ & $1.4(2)$ & & \\
\hline $\mathrm{C}(15)-\mathrm{N}(2)-\mathrm{C}(3)-\mathrm{N}(1)$ & $-168.86(16)$ & & \\
\hline $\mathrm{C}(5)-\mathrm{N}(2)-\mathrm{C}(3)-\mathrm{Pd}$ & $-173.54(14)$ & & \\
\hline $\mathrm{C}(15)-\mathrm{N}(2)-\mathrm{C}(3)-\mathrm{Pd}$ & $16.2(3)$ & & \\
\hline $\mathrm{O}(1)-\mathrm{Pd}-\mathrm{C}(3)-\mathrm{N}(1)$ & $-39.85(15)$ & & \\
\hline $\mathrm{O}(1) \# 1-\mathrm{Pd}-\mathrm{C}(3)-\mathrm{N}(1)$ & $140.15(15)$ & & \\
\hline C(3)\#1-Pd-C(3)-N(1) & $83(53)$ & & \\
\hline $\mathrm{O}(1)-\mathrm{Pd}-\mathrm{C}(3)-\mathrm{N}(2)$ & $133.99(15)$ & & \\
\hline O(1)\#1-Pd-C(3)-N(2) & $-46.01(15)$ & & \\
\hline C(3)\#1-Pd-C(3)-N(2) & $-103(53)$ & & \\
\hline $\mathrm{C}(3)-\mathrm{N}(1)-\mathrm{C}(4)-\mathrm{C}(5)$ & $0.2(2)$ & & \\
\hline $\mathrm{C}(6)-\mathrm{N}(1)-\mathrm{C}(4)-\mathrm{C}(5)$ & $-175.80(18)$ & & \\
\hline $\mathrm{N}(1)-\mathrm{C}(4)-\mathrm{C}(5)-\mathrm{N}(2)$ & $0.6(2)$ & & \\
\hline $\mathrm{C}(3)-\mathrm{N}(2)-\mathrm{C}(5)-\mathrm{C}(4)$ & $-1.3(2)$ & & \\
\hline $\mathrm{C}(15)-\mathrm{N}(2)-\mathrm{C}(5)-\mathrm{C}(4)$ & $169.56(17)$ & & \\
\hline $\mathrm{C}(3)-\mathrm{N}(1)-\mathrm{C}(6)-\mathrm{C}(7)$ & $117.74(19)$ & & \\
\hline $\mathrm{C}(4)-\mathrm{N}(1)-\mathrm{C}(6)-\mathrm{C}(7)$ & $-66.9(2)$ & & \\
\hline $\mathrm{C}(3)-\mathrm{N}(1)-\mathrm{C}(6)-\mathrm{C}(11)$ & $-66.3(2)$ & & \\
\hline $\mathrm{C}(4)-\mathrm{N}(1)-\mathrm{C}(6)-\mathrm{C}(11)$ & 109.1(2) & & \\
\hline $\mathrm{C}(11)-\mathrm{C}(6)-\mathrm{C}(7)-\mathrm{C}(8)$ & $2.2(3)$ & & \\
\hline $\mathrm{N}(1)-\mathrm{C}(6)-\mathrm{C}(7)-\mathrm{C}(8)$ & $177.94(16)$ & & \\
\hline $\mathrm{C}(11)-\mathrm{C}(6)-\mathrm{C}(7)-\mathrm{C}(12)$ & $-176.61(17)$ & & \\
\hline $\mathrm{N}(1)-\mathrm{C}(6)-\mathrm{C}(7)-\mathrm{C}(12)$ & $-0.8(3)$ & & \\
\hline $\mathrm{C}(6)-\mathrm{C}(7)-\mathrm{C}(8)-\mathrm{C}(9)$ & $-0.9(3)$ & & \\
\hline $\mathrm{C}(12)-\mathrm{C}(7)-\mathrm{C}(8)-\mathrm{C}(9)$ & $177.86(19)$ & & \\
\hline $\mathrm{C}(7)-\mathrm{C}(8)-\mathrm{C}(9)-\mathrm{C}(10)$ & $-0.3(3)$ & & \\
\hline $\mathrm{C}(7)-\mathrm{C}(8)-\mathrm{C}(9)-\mathrm{C}(13)$ & $-179.31(19)$ & & \\
\hline $\mathrm{C}(8)-\mathrm{C}(9)-\mathrm{C}(10)-\mathrm{C}(11)$ & $0.3(3)$ & & \\
\hline$C(13)-C(9)-C(10)-C(11)$ & $179.36(17)$ & & \\
\hline $\mathrm{C}(9)-\mathrm{C}(10)-\mathrm{C}(11)-\mathrm{C}(6)$ & $0.8(3)$ & & \\
\hline $\mathrm{C}(9)-\mathrm{C}(10)-\mathrm{C}(11)-\mathrm{C}(14)$ & $-177.45(17)$ & & \\
\hline $\mathrm{C}(7)-\mathrm{C}(6)-\mathrm{C}(11)-\mathrm{C}(10)$ & $-2.1(2)$ & & \\
\hline $\mathrm{N}(1)-\mathrm{C}(6)-\mathrm{C}(11)-\mathrm{C}(10)$ & $-177.88(14)$ & & \\
\hline$C(7)-C(6)-C(11)-C(14)$ & 176.14(17) & & \\
\hline $\mathrm{N}(1)-\mathrm{C}(6)-\mathrm{C}(11)-\mathrm{C}(14)$ & $0.4(2)$ & & \\
\hline $\mathrm{C}(3)-\mathrm{N}(2)-\mathrm{C}(15)-\mathrm{C}(20)$ & $-87.7(2)$ & & \\
\hline $\mathrm{C}(5)-\mathrm{N}(2)-\mathrm{C}(15)-\mathrm{C}(20)$ & $102.9(2)$ & & \\
\hline $\mathrm{C}(3)-\mathrm{N}(2)-\mathrm{C}(15)-\mathrm{C}(16)$ & $95.0(2)$ & & \\
\hline $\mathrm{C}(5)-\mathrm{N}(2)-\mathrm{C}(15)-\mathrm{C}(16)$ & $-74.3(2)$ & & \\
\hline $\mathrm{C}(20)-\mathrm{C}(15)-\mathrm{C}(16)-\mathrm{C}(17)$ & $1.1(3)$ & & \\
\hline $\mathrm{N}(2)-\mathrm{C}(15)-\mathrm{C}(16)-\mathrm{C}(17)$ & $178.34(15)$ & & \\
\hline $\mathrm{C}(20)-\mathrm{C}(15)-\mathrm{C}(16)-\mathrm{C}(21)$ & $-178.94(17)$ & & \\
\hline $\mathrm{N}(2)-\mathrm{C}(15)-\mathrm{C}(16)-\mathrm{C}(21)$ & $-1.7(2)$ & & \\
\hline $\mathrm{C}(15)-\mathrm{C}(16)-\mathrm{C}(17)-\mathrm{C}(18)$ & $-1.0(3)$ & & \\
\hline $\mathrm{C}(21)-\mathrm{C}(16)-\mathrm{C}(17)-\mathrm{C}(18)$ & 179.07(18) & & \\
\hline $\mathrm{C}(16)-\mathrm{C}(17)-\mathrm{C}(18)-\mathrm{C}(19)$ & $0.8(3)$ & & \\
\hline $\mathrm{C}(16)-\mathrm{C}(17)-\mathrm{C}(18)-\mathrm{C}(22)$ & $-178.29(19)$ & & \\
\hline
\end{tabular}


Symmetry transformations used to generate equivalent atoms:

$\# 1-\mathrm{x},-\mathrm{y},-\mathrm{z}$

\section{Acknowledgement}

The authors thank the National Science Foundation (grant CHE-9709005) and the University of Wisconsin for funds to purchase the diffractometer and computers. 